\title{
QUANTIZATION OF LIE BIALGEBRAS VIA THE FORMALITY OF THE OPERAD OF LITTLE DISKS
}

\author{
DIMITRI TAMARKIN
}

\begin{abstract}
We give a proof of Etingof-Kazhdan theorem on quantization of Lie bialgebras based on the formality of the chain operad of little disks and show that the Grotendieck-Teichmüller group acts non-trivially on the corresponding quantization functors.
\end{abstract}

\section{INTRODUCTION}

1.1. The present paper is an improved and enlarged version of [18]. We give a proof of EtingofKazhdan theorem on quantization of Lie bialgebras based on the formality of the chain operad of little disks.

Any known construction of such a formality involves multiple zeta values; in particular there is no canonical way to establish such a formality over $\mathbb{Q}$. For example, in the construction from [15] one needs to choose an associator over $\mathbb{Q}$. In $[16]$ it is shown that different associators produce homotopically non-equivalent formalities of chain operad of little disks. Each of these formalities, in turn, produces a certain quantization procedure of Lie bialgebras and we prove that these procedures are not isomorphic. This can be considered as a step in studying the action of GrotendieckTeichmüller group on quantization functors originated in [7].

\subsection{Idea of the construction of quantization.}

1.2.1. For simplicity, let us work in the category of $A$-modules, where $A$ is a commutative $\mathbb{Q}$ algebra. Let $\mathfrak{g}$ be a Lie bialgebra with bracket [,] and cobracket $\delta$. Call $\mathfrak{g}$ conilpotent if for any $x \in \mathfrak{g}$ there exists an $N$ such that any $N$-fold iteration of $\delta$ applied to $x$ produces zero.

1.2.2. Let $H$ be a Hopf algebra with product $\cdot$, coproduct $\Delta$, unit 1 , and counit $\epsilon$ (we do not assume that the antipode exists). Let $\Delta^{\prime}(x)=\Delta(x)-1 \otimes x-x \otimes 1$. Call $H$ conilpotent if for any $x$ such that $\epsilon(x)=0$ there exists an $N$ such that any $N$-fold iteration of $\Delta^{\prime}$ applied to $x$ produces zero.

1.2.3. Note that in any conilpotent $H$ there exists an antipode map and it is uniquely defined.

1.2.4. We are going to construct a functor $\mathbf{Q}$ from the category of conilpotent Lie bialgebras to the category of conilpotent Hopf algebras

PARTIALLY SUPPORTED BY AN NSF GRANT AND A. SLOAN RESEARCH FELLOWSHIP 
1.2.5. How this implies the Etingof-Kazhdan quantization theorem? This theorem, given a Lie bialgebra $\mathfrak{g}$ over $\mathbb{Q}$, produces a deformed Hopf algebra structure on $U(\mathfrak{g})[[t]](U(\mathfrak{g})$ is the universal enveloping algebra of the Lie algebra $\mathfrak{g} ; t$ is a formal parameter; the Hopf algebra structure is in the symmetric monoidal category of topologically free and complete $k[[t]]$-modules; this Hopf structure reduces $(\bmod t)$ to that on $U(\mathfrak{g})$.) In our language this will look as follows. Set $\mathfrak{a}_{n}:=\mathbb{Q}[t] /\left(t^{n}\right)$ and let $C_{n}$ be the category of $\mathfrak{a}_{n}$-modules. For every $n$ we have an obvious map $p_{n}: \mathfrak{a}_{n} \rightarrow \mathfrak{a}_{n-1}$ which induces a functor $P_{n}: C_{n} \rightarrow C_{n-1}$ (and $C_{n}, P_{n}$ form a projective system of categories so that the projective two-limit $\operatorname{liminv}_{n} C_{n}$ is the category of complete $\mathbb{Q}[[t]]$-modules and their morphisms.)

Let $\mathfrak{g}_{n}$ be the Lie bialgebra over $\mathfrak{a}_{n}$ defined as follows:

1) $\mathfrak{g}_{n}=\mathfrak{g} \otimes_{\mathbb{Q}} \mathfrak{a}_{n}$

2) the bracket is induced by that on $\mathfrak{g}$;

3 ) the cobracket $\delta_{n}$ on $\mathfrak{g}_{n}$ is given by:

$$
\delta_{n}(x \otimes a)=\operatorname{ta} \delta(x),
$$

where $x \in \mathfrak{g}, a \in \mathfrak{a}_{n}$, and $\delta$ is the cobracket in $\mathfrak{g}$.

Then $\mathfrak{g}_{n}$ is conilpotent and we have natural identifications $P_{n}\left(\mathfrak{g}_{n}\right) \cong \mathfrak{g}_{n-1}$.

Our quantization functor will then produce a conilpotent Hopf algebra $H_{n}:=\mathbf{Q}\left(\mathfrak{g}_{n}\right)$ over $\mathfrak{a}_{n}$. As our quantization is functorial, we will have identifications $P_{n}\left(H_{n}\right) \rightarrow H_{n-1}$; the Etingof-Kazhdan quantized Hopf algebra will be given by $\operatorname{liminv}_{n} H_{n}$.

1.2.6. Constructing the quantization. Let $\mathfrak{g}$ be a conilpotent Lie bialgebra. Let $C^{\bullet}(\mathfrak{g})$ be its cochain complex with respect to the cobracket. This means that $C^{\bullet}(\mathfrak{g})=S(\mathfrak{g}[-1])$ is a free graded commutative algebra equipped with a differential $D$ defined on the space of generators $\mathfrak{g}[-1] \subset S(\mathfrak{g}[-1])$ by the cobracket $\delta: \mathfrak{g}[-1] \rightarrow S^{2}(\mathfrak{g}[-1])$.

$C^{\bullet}(\mathfrak{g})$ has a structure of Gerstenhaber algebra so that the bracket on $\mathfrak{g}[-1] \subset C^{\bullet}(\mathfrak{g})$ is defined by the bracket on $\mathfrak{g}$.

1.2.7. Let ger be the operad of Gerstenhaber algebras and braces be the operad of brace structures (see [9],[8], see also Sec. 5.1). Let hoger $\rightarrow$ ger be the standard resolution of ger (as defined in [9], see also [11]). It is shown [13] that the operads braces and ger are quasi-isomorphic. This means that there exists a quasi-isomorphic map of operads hoger $\rightarrow$ braces. Therefore, there is a way to construct a brace-algebra out of a Gerstenhaber algebra. Denote this way by $W$ (it is a functor from the category of Gerstenhaber algebras to the category of brace algebras. Thus, $W\left(C^{\bullet}(\mathfrak{g})\right)$ is a brace algebra.

1.2.8. Remark 1. It is exactly on this step that the associators or integrals are being used.

Remark 2. One of the steps of the proof of the formality of braces in [13] is linking braces with the operad of singular chains of the operad of little disks (this step is purely "combinatorial",- - it does not use transcendental methods). Thus, the formality of braces follows from the formality of the operad of singular chains of the operad of little disks (see. [12], [15]).

1.2.9. As follows from the definitions, given a brace algebra $A$, one has a canonical Hopf algebra structure on the co-free coalgebra

$$
\bigoplus_{n=0}^{\infty} A[1]_{2}^{\otimes n}
$$


Denote this Hopf algebra by $H(A)$. Thus $h(\mathfrak{g})=H W C^{\bullet}(\mathfrak{g})$ is a differential graded Hopf algebra. If $H^{\neq 0} h(\mathfrak{g})=0$, one gets an induced Hopf algebra structure on $H^{0} h(\mathfrak{g})$ which is the quantization $\mathbf{Q}(\mathfrak{g})$ of $\mathfrak{g}$.

This is just an idea of our approach. Actually, we will carry out this program on the so called universal level, similar to [6].

1.3. Universal language. As was explained in [6], a more appropriate way to deal with quantization of Lie bialgebras/dequantization of bialgebras is via the universal language of PROPs.

The language of PROPs is designed in order to describe algebraic structures on an object $A$ which include maps $A^{\otimes m} \rightarrow A^{\otimes n}$ where $m, n$ can be any non-negative integers. Recall that in a simpler situation, when all structure maps are of the type $A^{\otimes m} \rightarrow A$ a simpler language, namely that of operads, can be used. Unfortunately, bialgebras are clearly not of this type, that's why we have to use PROPs. Thus, there are PROP's LBA, BA of Lie bialgebras and bialgebras.

We have an additional subtlety: we have to deal with conilpotent bialgerbas. We will see that the conilpotency can be adequately expressed in terms of certain completions of the PROPs LBA and BA. A similar approach is used in [6].

More precisely, we define projective systems of PROP's

$$
\begin{gathered}
\cdots \rightarrow \mathbf{L B A}_{n} \rightarrow \mathbf{L B A}_{n-1} \rightarrow \cdots \rightarrow \mathbf{L B A}_{1} ; \\
\quad \cdots \rightarrow \mathbf{B A}_{n} \rightarrow \mathbf{B A}_{n-1} \rightarrow \cdots \rightarrow \mathbf{B A}_{1}
\end{gathered}
$$

where $\mathbf{L B A} \mathbf{A}_{n}$ are quotients of $\mathbf{L B A}$ with respect to a decreasing chain of ideals $\mathcal{I}_{1} \supset \mathcal{I}_{2} \supset \cdots$;

$$
\mathbf{L B} \mathbf{A}_{n}=\mathbf{L B A} / \mathcal{I}_{n}
$$

and likewise for $\mathbf{B} \mathbf{A}_{n}$ (see 3.1.5).

Next we use Ioneda's embeddings $\mathbf{L B} \mathbf{A}_{n} \rightarrow \mathbf{L B} \mathbf{A}_{n}^{\wedge} ; \mathbf{B} \mathbf{A}_{n} \rightarrow \mathbf{B} \mathbf{A}_{n}^{\wedge}$, where $\mathbf{L B} \mathbf{A}_{n}^{\wedge}$ is the category of finitely generated functors from the category $\mathbf{L B} \mathbf{A}_{n}$ to the category of vector spaces, and likewise for $\mathbf{B} \mathbf{A}_{n}$. The categories $\mathbf{L B} \mathbf{A}_{n}^{\wedge}, \mathbf{B} \mathbf{A}_{n}^{\wedge}$ inherit a symmetric monoidal structure from $\mathbf{L} \mathbf{B} \mathbf{A}_{n}^{\wedge}, \mathbf{B} \mathbf{A}_{n}^{\wedge}$, and we can also construct projective systems of symmetric monoidal categories

$$
\begin{gathered}
\cdots \rightarrow \mathbf{L B A}_{n}^{\wedge} \rightarrow \mathbf{L B A}_{n-1}^{\wedge} \rightarrow \cdots \rightarrow \mathbf{L B A}_{1}^{\wedge} \\
\cdots \rightarrow \mathbf{B A}_{n}^{\wedge} \rightarrow \mathbf{B A}_{n-1}^{\wedge} \rightarrow \cdots \rightarrow \mathbf{B A}_{1}^{\wedge} .
\end{gathered}
$$

We show (Theorem 3.3) that these systems are equivalent: there exist symmetric monoidal equivalences

$$
\mathbf{B A}_{n}^{\wedge} \rightarrow \mathbf{L B A}_{n}^{\wedge}
$$

compatible with the functors $\mathbf{L B} \mathbf{A}_{n}^{\wedge} \rightarrow \mathbf{L B} \mathbf{A}_{n-1}^{\wedge} ; \mathbf{B A}_{n}^{\wedge} \rightarrow \mathbf{B A}_{n-1}^{\wedge}$.

We also show that this theorem gives us a way to quantize conilpotent Lie algebras:

given a k-linear SMC $\mathcal{C}$ satisfying certain restrictions (Sec. 4.1), we make a definition of a conilpotent Lie bialgebra and a conilpotent bialgebra in $\mathcal{C}$ (see 4.1.2), and construct an equivalence of the category of conilpotent Lie bialgebras in $\mathcal{C}$ and the category of conilpotent bialgebras in $\mathcal{C}$.

In the case when $\mathcal{C}$ is the category of $A$-modules, where $A$ is a commutative $\mathbf{k}$-algebra, the abstract definition of a conilpotent bialgebra and Lie bialgebra in $\mathcal{C}$ as in 4.1 .2 is equivalent to that in 1.2 .

After having established the quantization procedure, we investigate how it depends on the choice of a zigzag quasi-isomorphism between the operad of braces and an operad of Gerstenhaber algebras. In the last section of the paper we study this dependence, and show that it is essential: 
we prove that the quantization functors produced using different quasi-isomorphisms between the operads of braces and Gerstenhaber algebras are isomorphic if and only if the quasi-isomorphisms are homotopy equivalent (Theorem 8.1). Furthermore, we show that given a pair $q_{1}, q_{2}$ of homotopy inequivalent quasi-isomorphisms between the operads of braces and Gerstenhaber algebras, there exists a co-nilpotent Lie bialgebra in a certain symmetric monoidal category $C$ such that its quantizations using $q_{1}$ and $q_{2}$ produce non-isomorphic bialgebras in $C$.

Lastly we investigate the relationship with associators. It turns out that given an associator, there is a natural way to construct a zigzag quasi-isomorphism between the operad of Gerstenhaber algebras and the operad of braces, hence a quantization procedure. We show that different associators produce homotopy non-equivalent quasi-isomorphisms between the operads, hence nonisomorphic quantization procedures. This concludes the paper.

1.4. Plan of the paper. In Section 3 we provide all the necessary material in order to formulate the universal quantization Theorem 3.3. This includes a discussion of PROP's of bialgebras and Lie bialgebras. Next, we define projective systems formed by the $\mathrm{SMC} \mathbf{L B} \mathbf{A}_{n}, \mathbf{B} \mathbf{A}_{n}$ and their Ioneda's completions. This allows us to formulate the quantization theorem 3.3 in the universal language.

We postpone the proof of this result, showing instead that this universal quantization result allows one to quantize conilpotent Lie bialgebras (Section 4). We make an abstract definition of a conilpotent Lie bialgebra and a bialgebra and show that Theorem 3.3 defines a quantization in this setting.

Next, we prove Theorem 3.3. The proof occupies Sections 5-7. A plan of the proof can be found in Sec. 5, so we do not discuss it here, instead only making a remark that, essentially, we translate the sketch in Sec. 1.2 into the universal language.

The last Section 8 deals with dependence of the quantization functor on the choice of a quasiisomorphism between the operads of braces and Gerstenhaber algebras.

There are three Appendices. in Appendix 1 we collect some categorical constructions which are used throughout the paper. In Appendix 2 we study the automorphism group of the operad hoger in the derived category of dg-operads. In Appendix 3 we collected all the information on the Grothendieck-Teichmuller group which is used in this paper.

\section{Notation}

We fix a ground field $\mathbf{k}$ of characteristic 0 .

Throughout the paper we use abbreviations SM for "symmetric monoidal" and SMC for "symmetric monoidal category.

Mostly, operads are denoted by words in lower case boldface, for example:

lie is the operad of Lie algebras;

ger is the operad of Gerstenhaber algebras;

hoger $\rightarrow$ ger is the standard cofibrant resolution of ger;

braces is the operad of brace algebras.

PROPs are mostly denoted unsing the upper case bold face:

ASSOC is the PROP of associative algebras;

COASS $=$ ASSOC $^{\text {op }}$ is the PROP of co-associative co-algebras;

LIE is the PROP of Lie algebras; COLIE $=\mathbf{L I E}^{\text {op }}$ is the PROP of Lie coalgebras;

GER is the PROP of Gerstenhaber algebras;

HOGER is the PROP generated by the operad hoger; 
BRACES is the PROP of brace algebras;

LBA is the PROP of Lie bialgebras;

BA is a PROP such that a BA-algebra structure on an object $\mathfrak{m}$ in a SMC $\mathcal{C}$ is equivalent to a bialgebra structure on $\mathbf{1} \oplus m$ with the standard unit and counit map. Here $\mathbf{1}$ is the tensor unit on $\mathcal{C}$, and we assume that $\mathbf{1} \oplus \mathfrak{m}$ exists.

Objects of PROPs are denoted by $\langle n>$, where $n=1,2,3, \ldots$ so that $<m>\otimes<n>=<$ $m+n>$. The reason for using the curly brackets is that the square brackets are used to denote the shift in cohomological degree.

Given an object $X$ in a $\operatorname{SMC} \mathcal{C}$ we denote by full $(X)$ its full operad and by $\operatorname{FULL}(X)$ its full PROP.

\section{Formulating the universal Statement}

\subsection{Prop of bialgebras.}

3.1.1. Let $C$ be a dg SM category with unit $\mathbf{1}$ and finite direct sums. We say that $X \in C$ has a structure of bialgebra if $C$ has structure of associative algebra with unit and a coassociative coalgebra with counit such that the coproduct map $X \rightarrow X \otimes X$ and the counit map $X \rightarrow \mathbf{1}$ are morphisms of unital associative algebras.

3.1.2. We say that $m \in C$ has a BA-structure if $X:=\mathbf{1} \oplus m$ exists and we have a bialgebra structure on $X$ with the natural inclusion of $\mathbf{1}$ being the unit and the natural projection onto 1 being the counit.

3.1.3. There exists a PROP BA of BA-algebras. BA is uniquely specified by the condition that BA-structures on $\mathfrak{m} \in C$ are in 1-1 correspondence with the maps of PROPs BA $\rightarrow$ FULL(m)

Note that we can now talk about BA -algebras in any SMC regardless of existence of direct sums.

3.1.4. A BA-structure on $\mathfrak{m}$ implies structures of associative algebra without unit and of a coassociative algebra without unit on $\mathfrak{m}$. Let ASSOC (resp. COASS) be the PROPs describing associative algebra (resp. coassociative coalgebra) structure. We thus have maps of PROPs $i: \mathbf{A S S O C} \rightarrow \mathbf{B A}$ and $j: \mathbf{C O A S S} \rightarrow \mathbf{B A}$. The composition defines a map

$$
\phi_{y}: \operatorname{ASSOC}(<y>,<z>) \otimes_{S_{y}} \operatorname{COASS}(<x>,<y>) \rightarrow \operatorname{BA}(<x>,<z>) .
$$

Therefore, we have a map

$$
\phi:=\sum \phi_{n}: \bigoplus_{n=x}^{\infty} \operatorname{ASSOC}(<n>,<z>) \otimes_{S_{n}} \operatorname{COASS}(<x>,<n>)
$$

$$
\rightarrow \mathbf{B A}(<x>,<z>)
$$

PROPOSITION 3.1. ( [7], [14]) $\phi$ is an isomorphism 
3.1.5. Let $\mathcal{I}_{n} \subset \mathbf{B A}$ be the double-sided categorical ideal generated by $\operatorname{Id}_{<m>}$ for all $m>n$. Let $\mathbf{B A}_{n}:=\mathbf{B A} / \mathcal{I}_{n}$. This readily implies that $\operatorname{hom}_{\mathbf{B A}_{n}}(<p>;<q>)=0$ as long as $p>n$ or $q>n$ so that all $\langle p\rangle$ with $p>n$ are isomorphic to the zero-object.

If $p, q \leq n$, then we have:

(1) $\mathbf{B A}_{n}(<p>,<q>)$

$$
\cong \bigoplus_{r=\max (p, q)}^{n} \operatorname{COASS}(<p>,<r>) \otimes_{S_{r}} \operatorname{ASSOC}(<r>,<q>) .
$$

Note that the ideals $\mathcal{I}_{n}$ are actually tensor ideals meaning that whenever $f \in \mathcal{I}_{n}$ and $g$ is any arrow, $f \otimes g \in \mathcal{I}_{n}$. Therefore, we have an induced tensor structure on $\mathbf{B} \mathbf{A}_{n}$.

Since the ideals $\mathcal{I}_{n}$ form a decreasing chain, the quotients form a projective system

$$
\mathbf{B A} \rightarrow \cdots \rightarrow \mathbf{B A}_{n} \rightarrow \mathbf{B A}_{n-1} \rightarrow \cdots \rightarrow \mathbf{B A}_{1}
$$

in which every arrow is a symmetric monoidal functor.

We will use the following notation for these arrows:

$$
P_{n m}: \mathbf{B A}_{n} \rightarrow \mathbf{B A}_{m} ; P_{n}: \mathbf{B A} \rightarrow \mathbf{B A}_{n} .
$$

We can form a topological PROP $\operatorname{liminv}_{n} \mathbf{B} \mathbf{A}_{n}$ so that the map

$$
\mathbf{B A} \rightarrow \operatorname{liminv}_{n} \mathbf{B A}_{n}
$$

can be viewed as a completion of BA. However, our results will be formulated on the level of the projective system of PROPs $\mathbf{B A}_{n}$ without passing to the projective limit; we thus won't discuss this projective limit in detail.

3.1.6. Ioneda's completions. Given a finite $k$-linear category $\mathcal{C}$ (see Appendix 1 ) we denote by $\mathcal{C}^{\wedge}$ the abelian category of functors $\mathcal{C}^{\mathrm{op}} \rightarrow$ vect $_{\text {fin }}$, where vect $_{\text {fin }}$ is the category of finite-dimensional $\mathbf{k}$-vector spaces.

Suppose that $\mathcal{C}$ is a SMC. We then have an induced SM-structure on $\mathcal{C}^{\wedge}$ (see Appendix 1).

We have functors

$$
\begin{gathered}
P_{n m}^{-1}: \mathbf{B A}_{m}^{\wedge} \rightarrow \mathbf{B} \mathbf{A}_{n}^{\wedge} ; \\
P_{n m !}: \mathbf{B A}_{n}^{\wedge} \rightarrow \mathbf{B A}_{m}^{\wedge},
\end{gathered}
$$

the latter functor has a symmetric monoidal structure (these functors are defined in the Appendix).

3.2. A similar story takes place in the world of Lie bialgebras. We have a PROP LBA of Lie bialgebras.

3.2.1. Let LBA be the PROP of Lie bialgebras, LIE be the PROP of Lie algebras and COLIE be the PROP of Lie coalgebras. We have maps $i:$ LIE $\rightarrow$ LBA and $j:$ COLIE $\rightarrow$ LBA.

PROPOSITION 3.2. The natural map $\phi: \oplus_{n \geq|Y|} \mathbf{C O L I E}(<y>,<n>) \otimes_{S_{n}} \mathbf{L I E}(<n>,<$ $x>) \rightarrow \mathbf{L B A}(<y>,\langle x\rangle)$ is an isomorphism. 
3.2.2. Let $\mathcal{I}_{n} \subset$ LBA be the double-sided ideal generated by $\operatorname{Id}_{<N>}$ for all $N>n$. We then see that this ideal is also a symmetric monoidal ideal.

We set $\mathbf{L B} \mathbf{A}_{n}:=\mathbf{L B A} / \mathcal{I}_{n}$. We then have

$$
\mathbf{L B A}_{n}(<k>,<l>)=0
$$

as long as $k>n$ or $l>n$.

In the case $k, l \leq n$, we have

$$
\mathbf{L B A}_{n}(<k>,<l>) \cong \bigoplus_{m=k}^{n} \mathbf{C O L I E}(<k>,<m>) \otimes_{S_{m}} \mathbf{L I E}(<m>,<l>) .
$$

The symmetric monoidal categories $\mathbf{L B} \mathbf{A}_{n}$ form a projective system

$$
\mathbf{L B A} \rightarrow \cdots \rightarrow \mathbf{L B A}_{n} \rightarrow \mathbf{L B A}_{n-1} \cdots \rightarrow \mathbf{L B A}_{1},
$$

where all arrows have a natural symmetric monoidal structure.

We denote the arrows in this system as follows:

$$
P_{n m}: \mathbf{L B A}_{n} \rightarrow \mathbf{L B A}_{m} ; P_{n}: \mathbf{L B A} \rightarrow \mathbf{L B A}_{n}
$$

We can form a completion

$$
\mathbf{L B A} \rightarrow \operatorname{liminv}_{n} \mathbf{L B A}_{n},
$$

but mostly, we won't pass to the limit.

We have functors

$$
P_{n m}^{-1}: \mathbf{L B A}_{m}^{\wedge} \rightarrow \mathbf{L B A}_{n}^{\wedge}
$$

and

$$
P_{n m !}: \mathbf{L B A}_{n}^{\wedge} \rightarrow \mathbf{L B A}_{m}^{\wedge}
$$

the latter functor has a symmetric monoidal structure (Appendix 1).

3.3. Universal quantization theorem. We have a pair of projective systems of SMC: the first one is

$$
\cdots \rightarrow \mathbf{B A}_{n}^{\wedge} \rightarrow \mathbf{B A}_{n-1}^{\wedge} \rightarrow \cdots
$$

the second one is

$$
\cdots \rightarrow \mathbf{L B A}_{n}^{\wedge} \rightarrow \mathbf{L B A}_{n-1}^{\wedge} \rightarrow \cdots
$$

We are going to formulate the theorem saying that the two systems are equivalent. Let us first define the notion of equivalence of projective systems of SMC.

3.3.1. Equivalence of projective systems of SMC. We define a projective system of $S M C$ as a collection of $\mathrm{SMC} \mathcal{C}_{n}, n=1,2, \cdots$, and symmetric monoidal functors

$$
p_{n}: \mathcal{C}_{n} \rightarrow \mathcal{C}_{n-1}, n>1 .
$$

In order to denote projective systems of SMC, we will use underlined symbols or words. For example, let us denote the projective system we have just introduced by $\underline{\mathcal{C}}$.

Let

$$
\cdots \stackrel{p_{n+1}^{\prime}}{\rightarrow} \mathcal{C}_{n}^{\prime} \stackrel{p_{n}^{\prime}}{\rightarrow} \cdots
$$

be another projective system of SMC, and denote it by $\underline{\mathcal{C}^{\prime}}$. We define a functor $F: \underline{\mathcal{C}} \rightarrow \underline{\mathcal{C}^{\prime}}$ as

- a collection of SM functors $F_{n}: \mathcal{C}_{n} \rightarrow \mathcal{C}_{n}^{\prime}$; 
— a collection of isomorphisms of SM functors

$$
p_{n}^{\prime} F_{n} \rightarrow F_{n-1} p_{n}
$$

for all $n>1$.

We say that $F$ is an equivalence if each $F_{n}$ is an equivalence of categories.

3.3.2. Let $\underline{\mathbf{B A}} ; \underline{\mathbf{B A}}^{\wedge} ; \underline{\mathbf{L B A}} ; \underline{\mathbf{L B A}}{ }^{\wedge}$ be the projective systems formed by $\mathbf{B} \mathbf{A}_{n}, \mathbf{B} \mathbf{A}_{n}^{\wedge}, \mathbf{L B} \mathbf{A}_{n}$, and $\mathbf{L B A}_{n}^{\wedge}$ respectively.

3.3.3. 2-category. This subsection won't be needed for the formulating of our result.

Given a pair of functors $F, G: \underline{\mathcal{C}} \rightarrow \underline{\mathcal{C}^{\prime}}$ we can define the notion of a map $\alpha: F \rightarrow G$ as a collection of SM natural transformations

$$
\alpha_{n}: F_{n} \rightarrow G_{n}
$$

which commute with $p_{n}, p_{n}^{\prime}$ in the obvious way.

We thus get a set $\operatorname{hom}(F, G)$ of all maps $F \rightarrow G$. It is clear that this way the functors $\mathcal{C} \rightarrow \mathcal{C}^{\prime}$ form a category, and all projective systems of SMC form a 2-category.

3.3.4. Invertibility of equivalences. Given a SM equivalence $F: C_{1} \rightarrow C_{2}$ of SMC $C_{1}, C_{2}$, one can construct an inverse one $G: C_{2} \rightarrow C_{1}$ such that the compositions $F G: C_{2} \rightarrow C_{2} ; G F: C_{1} \rightarrow C_{1}$ are isomorphic to the Identity. Given another inverse to $F$, say $G_{1}$, we have a canonical isomorphism between $G$ and $G_{1}$.

Same holds true for equivalences of projective systems of SMC.

\subsubsection{Universal quantization theorem.}

THEOREM 3.3. There exists an equivalence

$$
\mathbf{Q}: \underline{\mathbf{B A}}^{\wedge} \rightarrow \underline{\mathbf{L B A}}^{\wedge}
$$

Note that, equivalently, one can say that there exists an equivalence in the opposite direction (according to 3.3.4).

Etingof-Kazhdan call Q "quantization", and the inverse equivalence "dequantization".

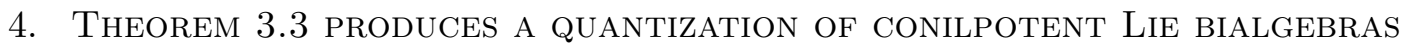

The plan is as follows:

1) We make a definition of a conilpotent Lie bialgebra/BA-algebra in a SMC $\mathcal{C}$ (provided that $\mathcal{C}$ satisfies certain conditions). Our ultimate goal is to construct an equivalence of the categories of conilpotent Lie bialgebras and BA-algebras in $\mathcal{C}$.

2) We relate the notions of conilpotent Lie bialgebra/ BA-algebra with the projective systems of SMC $\underline{\mathbf{L B A}}^{\wedge}$ and $\underline{\mathbf{B A}}^{\wedge}$. We do it as follows:

a) Let $\mathbf{L B A}_{n}^{\text {pro }} \subset \mathbf{B A}_{n}^{\wedge}$ be the full SM- subcategory of finitely-generated projective objects; let $\mathbf{B A}_{n}^{\text {pro }}$ be a similar thing, it is clear that $\mathbf{L B} \mathbf{A}_{n}^{\text {pro }}$; $\mathbf{B} \mathbf{A}_{n}^{\text {pro }}$ form projective sub-systems of SMC: $\underline{\mathbf{L B A}}^{\text {pro }} \subset \underline{\mathbf{L B A}}^{\wedge} ; \underline{\mathbf{B A}}^{\text {pro }} \subset \underline{\mathbf{B A}}^{\wedge}$ and that the quantization functor induces an equivalence of these sub-systems

$$
\mathrm{Q}: \underline{\mathrm{BA}}^{\text {pro }} \stackrel{\sim}{\rightarrow} \underline{\mathbf{L B A}}^{\text {pro }} .
$$

Indeed, any equivalence of abelian categories preserves the class of projective objects.

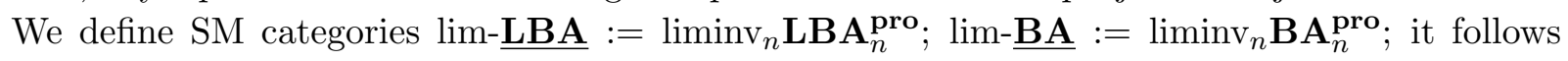
that $\mathbf{Q}$ induces an equivalence between these categories 
b) We show that the category of conilpotent Lie bialgebras in $\mathcal{C}$ is equivalent to the category of direct sum preserving SM-functors lim-LBA $\rightarrow \mathcal{C}$; likewise, the category of BA-algebras is equivalent to the category of direct sum preserving functors lim- $\underline{\mathbf{B A}} \rightarrow \mathcal{C}$.

3) As the equivalence $\mathbf{Q}: \lim -\underline{\mathbf{B A}} \rightarrow \lim -\underline{\mathbf{L B A}}$ happens to be direct sum preserving, it induces an equivalence functor from the category of conilpotent Lie bialgebras in $\mathcal{C}$ to the category of conilpotent BA-algebras in $\mathcal{C}$.

\subsection{Conilpotent Lie- and BA-algebras.}

4.1.1. Conditions on the $S M C$ we will work in. Let $\mathcal{C}$ be a $k$-linear symmetric monoidal category. We assume it possesses the following features:

1) Countable direct sums exist in $\mathcal{C}$ and are compatible with the tensor product: the natural map

$$
\bigoplus_{(i, j) \in I \times J} X_{i} \otimes Y_{j} \rightarrow\left(\bigoplus_{i \in I} X_{i}\right) \otimes\left(\bigoplus_{j \in J} Y_{j}\right)
$$

must be an isomorphism, where $I, J$ are at most countable sets, $X_{i}, i \in I ; Y_{j}, j \in J$ are arbitrary objects in $\mathcal{C}$.

2) We demand that for every object $Z \in \mathcal{C}$ and any at most countable family $\left\{X_{i}\right\}_{i \in I}$ in $\mathcal{C}$, the natural map

$$
\operatorname{hom}_{\mathcal{C}}\left(Z, \bigoplus_{i \in I} X_{i}\right) \rightarrow \prod_{i \in I} \operatorname{hom}_{\mathcal{C}}\left(Z, X_{i}\right)
$$

must be injective.

3) $\mathcal{C}$ must be closed under kernels of projectors.

We fix such a category $\mathcal{C}$.

4.1.2. Definition of a conilpotent Lie bialgebra in $\mathcal{C}$. Let $\mathfrak{g}$ be Lie bialgebra in $\mathcal{C}$. We then have natural maps $\operatorname{LBA}(<k>;<l>) \rightarrow \operatorname{hom}_{\mathcal{C}}\left(\mathfrak{g}^{\otimes k} ; \mathfrak{g}^{\otimes l}\right)$.

Let us now make a definition of a conilpotent Lie bialgebra. To this end, we need to introduce some notation. First of all, since finite direct sums exist in $\mathcal{C}$, given an object $X \in \mathcal{C}$ and a finite dimensional k-vector space $V$ we have a well defined notion of an object $V^{*} \otimes X$ it is defined as the representing object for the functor $h_{V^{*} \otimes X}: \mathcal{C}^{\text {op }} \rightarrow \operatorname{vect:~} Y \mapsto \operatorname{hom}_{\mathbf{k}}\left(V ; \operatorname{hom}_{\mathcal{C}}(Y ; X)\right)$. It is clear that $V^{*} \otimes X$ is isomorphic to the direct sum of $\operatorname{dim} V$ number of copies of $X$.

Next, we have a natural map of PROPs $j:$ COLIE $\rightarrow$ LBA (same as in Sec. 3.1.4), hence a map

$$
\operatorname{COLIE}(<k>;<l>) \rightarrow \operatorname{hom}_{\mathbf{k}}\left(\mathfrak{g}^{\otimes k} ; \mathfrak{g}^{\otimes l}\right) .
$$

Since the vector space on the LHS is finite dimensional, we get an induced element

$$
\delta_{k l} \in \operatorname{hom}\left(\mathfrak{g}^{\otimes k} ; \operatorname{COLIE}^{*}(<k>;<l>) \otimes \mathfrak{g}^{\otimes l}\right)
$$

where $\operatorname{COLIE}^{*}(<k>;<l>)$ is the dual vector space.

Furthermore, the symmetric group $S_{l}$ acts on the object $\operatorname{COLIE}^{*}(<k>;<l>) \otimes \mathfrak{g}^{\otimes l}$ by automorphisms. Let $P \in \mathbf{k}\left[S_{l}\right]$ be the standard averaging projector in the group algebra of $S_{l}$ :

$$
P=\frac{1}{l !} \sum_{\sigma \in S_{l}} \sigma
$$


Let $\left(\mathbf{C O L I E}^{*}(<k>;<l>) \otimes \mathfrak{g}^{\otimes l}\right)^{S_{l}}$ be the kernel of this projection The map $\delta_{k l}$ passes through this kernel so that we have a natural map

$$
\delta_{k l} \in \operatorname{hom}\left(\mathfrak{g}^{\otimes k} ;\left[\operatorname{COLIE}^{*}(<k>;<l>) \otimes \mathfrak{g}^{\otimes l}\right]^{S_{l}}\right) .
$$

We are now ready to make a definition of a conilpotent Lie bialgebra:

DEFINITION 4.1. A Lie bialgebra $\mathfrak{g} \in F^{1} \mathcal{C}$ is called conilpotent if for every $k$ there exists an element

$$
\delta_{k} \in \operatorname{hom}\left(\mathfrak{g}^{\otimes k} ; \bigoplus_{l=k}^{\infty}\left(\operatorname{COLIE}(<k>;<l>)^{*} \otimes \mathfrak{g}^{\otimes l}\right)^{S_{l}}\right)
$$

whose natural projections onto

$$
\operatorname{hom}\left(\mathfrak{g}^{\otimes k} ;\left(\mathbf{C O L I E}^{*}(<k>;<l>) \otimes \mathfrak{g}^{\otimes l}\right)^{S_{l}}\right)
$$

are $\delta_{k l}$.

Remarks 1. Such an element $\delta_{k}$, if exists, must be unique because of condition 2) from Sec. 4.1.

2. One can prove that it is sufficient to demand that $\delta_{1}$ exists, the existence of $\delta_{k}, k>1$ will then follow.

4.1.3. Conilpotent BA-algebras. Conilpotent BA-algebras in $\mathcal{C}$ are defined along the same lines as conilpotent Lie bialgebras.

Let $\mathfrak{m}$ be a BA-algebra in $\mathcal{C}$. We have natural maps

$$
\operatorname{COASS}(<k>;<l>) \rightarrow \mathbf{B A}(<k>;<l>) \rightarrow \operatorname{hom}_{\mathcal{C}}\left(\mathfrak{m}^{\otimes k} ; \mathfrak{m}^{\otimes l}\right)
$$

which can be rewritten as

$$
\Delta_{k, l}: \operatorname{hom}_{\mathcal{C}}\left(\mathfrak{m}^{\otimes k} ;\left(\operatorname{COASS}^{*}(<k>;<l>) \otimes \mathfrak{m}^{\otimes l}\right)^{S_{l}}\right)
$$

We then say that $\mathfrak{m}$ is conilpotent if for every $k$ there exists an element

$$
\Delta_{k} \in \operatorname{hom}_{\mathcal{C}}\left(\mathfrak{m}^{\otimes k} ; \bigoplus_{l}\left(\operatorname{COASS}^{*}(<k>;<l>) \otimes \mathfrak{m}^{\otimes l}\right)^{S_{l}}\right)
$$

such that its projection onto

$$
\operatorname{hom}_{\mathcal{C}}\left(\mathfrak{m}^{\otimes k} ;\left(\operatorname{COASS}^{*}(<k>;<l>) \otimes \mathfrak{m}^{\otimes l}\right)^{S_{l}}\right)
$$

is $\delta_{k l}$. It actually suffices to only check this condition for $k=1$.

4.2. Projective limits of $\mathbf{L B} \mathbf{A}_{n}^{\text {pro }} ; \mathbf{B A}_{n}^{\text {pro }}$. As explained above, we denote by

$$
\mathbf{L B A}_{n}^{\text {pro }} \subset \mathbf{L B A}_{n}^{\wedge} ; \quad \mathbf{B A}_{n}^{\text {pro }} \subset \mathbf{B} \mathbf{A}_{n}^{\wedge}
$$

the full subcategories of projective objects (they are automatically finitely generated).

The functors $P_{n m !}: \mathbf{L B} \mathbf{A}_{n}^{\wedge} \rightarrow \mathbf{L B A}_{m}^{\wedge}$ preserve the class of projective objects: indeed, they do clearly preserve free objects, hence they take any retraction of a free object to a retraction of a free object. Same is true for $P_{n m !}: \mathbf{B} \mathbf{A}_{n}^{\wedge} \rightarrow \mathbf{B} \mathbf{A}_{m}^{\wedge}$. One also sees that the class of projective objects is preserved by the tensor product in $\mathbf{L B} \mathbf{A}_{n}, \mathbf{B} \mathbf{A}_{n}$.

Therefore, the subcategories $\mathbf{L B} \mathbf{A}_{n}^{\text {pro }}$ form a projective subsystem of $\underline{\mathbf{L B A}}^{\wedge}$, denote this subsystem by $\underline{\mathbf{L B A}}^{\text {pro }} \subset \underline{\mathbf{L B A}}^{\wedge}$. In the same way, we get a subsystem $\underline{\mathbf{B A}}^{\text {pro }} \subset \underline{\mathbf{L B A}}^{\text {pro }}$. 
The functors $\mathbf{Q}_{n}: \mathbf{B A}_{n}^{\wedge} \rightarrow \mathbf{L B} \mathbf{A}_{n}^{\wedge}$ being equivalences of abelian categories must preserve the class of projective objects, hence we have an induced equivalence of systems:

$$
\mathrm{Q}: \underline{\mathrm{BA}}^{\text {pro }} \rightarrow \underline{\mathbf{L B A}}^{\text {pro }}
$$

4.2.1. We are going to define a symmetric monoidal category lim-LBA as the projective 2-limit of the projective system of SM-categories $\mathbf{L B} \mathbf{A}_{n}^{\text {pro }}$. The construction is the same as the construction of $\mathbb{Z}_{l}$-sheaves.

The definition is as follows.

An object $\underline{A}$ of lim- $\underline{\text { LBA }}$ is

a collection of objects $\underline{A}_{n} \in \mathbf{L B} \mathbf{A}_{n}^{\text {pro }}, n=1,2, \ldots$, and a collection of isomorphisms

$$
i_{n}: P_{n, n-1 !} \underline{A}_{n} \rightarrow \underline{A}_{n-1}, \quad n=2,3, \ldots
$$

Given $\underline{A}, \underline{B} \in \lim \underline{\text { LBA }}$, we define

$$
\operatorname{hom}(\underline{A} ; \underline{B}):=\operatorname{liminv}_{n} \operatorname{hom}_{\mathbf{L B A}}\left(\underline{A}_{n} ; \underline{B}_{n}\right),
$$

where the spaces $\operatorname{hom}_{\mathbf{L B A}}\left(A_{n}, B_{n}\right)$ form a projective system with the structure maps

$$
\operatorname{hom}_{\mathbf{L B A}}\left(A_{n}, B_{n}\right) \rightarrow \operatorname{hom}_{\mathbf{L B A}}{ }_{n-1}\left(\underline{A}_{n-1}, \underline{B}_{n-1}\right)
$$

defined by:

$$
\operatorname{hom}_{\mathbf{L B A}}\left(\underline{A}_{n}, \underline{B}_{n}\right) \stackrel{P_{n, n-1 !}}{\longrightarrow} \operatorname{hom}_{\mathbf{L B A}}\left(P_{n, n-1} ! \underline{A}_{n} ; P_{n, n-1 !} \underline{B}_{n}\right)=\operatorname{hom}_{\mathbf{L B A}}\left(\underline{A}_{n-1} ; \underline{B}_{n-1}\right) .
$$

This way, lim- $\underline{\text { LBA }}$ is a category.

Define the tensor product on lim- $\underline{\text { LBA }}$ componentwise:

$$
(\underline{A} \otimes \underline{B})_{n}:=\underline{A}_{n} \otimes \underline{B}_{n} .
$$

We see that lim-LBA is then naturally a SMC.

4.2.2. In a similar way, a SMC lim- $\underline{\mathbf{B A}}$ can be defined from the projective system $\underline{\mathbf{B A}}$. The equivalence $\mathbf{Q}$ induces an SM-equivalence $\lim -\underline{\mathbf{B A}} \rightarrow \lim -\underline{\mathbf{L B A}}$.

\subsection{Technical Lemmas concerning the categories lim-BA, lim-LBA.}

4.3.1. Direct sums in lim- $\underline{\mathbf{B A}}, \lim -\underline{\mathbf{L B A}}$. let $\mathbf{L}$ be either $\lim \underline{\mathbf{B A}}$ or $\lim -\underline{\mathbf{L B A}}$. For an object $\underline{A} \in \mathbf{L}$ let $|\underline{A}|$ be the minimal $n$ such that $\underline{A}_{n} \neq 0$. Call a family $\left\{\underline{A}^{i}\right\}_{i \in I}$ of objects in $\mathbf{L}$ admissible if for each $N$ there are only finitely many objects in this family with $\left|\underline{A}^{i}\right|<N$. In particular, our family must be at most countable.

LEMMA 4.2. There exist direct sums of admissible families. We have

$$
\left(\bigoplus_{i \in I} \underline{A}^{i}\right)_{n}=\bigoplus_{i \in I} \underline{A}_{n}^{i}
$$

where almost all terms on the RHS are zeros.

Proof. Clear

Call direct sums of the specified type admissible direct sums. 
LEMMA 4.3. Admissible direct sums are compatible with the tensor structure in the following sense: let $\left\{\underline{A}^{i}\right\}_{i \in I} ;\left\{\underline{B}^{j}\right\}_{j \in J}$ be admissible families in $\mathbf{L}$. Then the family

$$
\left\{\underline{A}^{i} \otimes \underline{B}^{j}\right\}_{(i, j) \in I \times J}
$$

is also admissible and the natural map

$$
\bigoplus_{(i, j) \in I \times J} \underline{A}^{i} \otimes \underline{B}^{j} \rightarrow\left(\bigoplus_{i \in I} \underline{A}^{i}\right) \otimes\left(\bigoplus_{j \in J} \underline{B}^{j}\right)
$$

is an isomorphism.

Proof. Indeed, admissibility of the family $\left\{\underline{A}^{i} \otimes \underline{B}^{j}\right\}_{(i, j) \in I \times J}$ follows from the equality $|\underline{A} \otimes \underline{B}|=$ $\max (|\underline{A}|,|\underline{B}|)$. The isomorphism follows from the comparison of the $n$-th components of both sides of (2).

LEMMA 4.4. The equivalence $\mathbf{Q}:$ lim- $\underline{\mathbf{B A}} \rightarrow$ lim-LBA preserves admissible direct sums: given an admissible family $\left\{\underline{A}^{i}\right\}_{i \in I}$ in lim-BA the family $\left\{\mathbf{Q}\left(\underline{A}^{i}\right)\right\}_{i \in I}$ is also admissible, and the natural map

$$
\bigoplus_{i \in I} \mathbf{Q}\left(\underline{A}^{i}\right) \rightarrow \mathbf{Q}\left(\bigoplus_{i \in I} \underline{A}^{i}\right)
$$

is an isomorphism

Proof. We have $|\mathbf{Q}(\underline{A})|=|\underline{A}|$, whence admissibility of $\left\{\mathbf{Q}\left(\underline{A}^{i}\right)\right\}_{i \in I}$. The isomorphism (3) can be easily checked by looking at the components of both sides.

4.3.2.

LEMMA 4.5. Let $p: \underline{R} \rightarrow \underline{P}$ be a component-wise surjective map of objects in lim-LBA or lim- $\underline{\mathbf{B A}}$. Then $p$ has a splitting $i: \underline{P} \rightarrow \underline{R}$.

Proof. We will prove Lemma for the category lim-LBA; the proof for lim- $\underline{\mathbf{B A}}$ is similar and omitted.

Let us construct the splittings $i_{n} \underline{P}_{n} \rightarrow \underline{R}_{n}$ by induction. Choose $i_{1}$ to be any splitting of the map $p_{1}$. Next, suppose that we have splittings $i_{m}: \underline{P}_{m} \rightarrow \underline{R}_{m}, m \leq n$, such that $P_{m, m-1} i_{m}=$ $i_{m-1} P_{m, m-1}$ for all $m \leq n$. Let us construct the splitting $i_{n+1}$. The splitting $i_{n}$ defines an identification $\underline{P}_{n}=\underline{R}_{n} \oplus \underline{K}_{n}$, where $\underline{K}_{n}:=\operatorname{Ker} p_{n}$. Choose any splitting of $p_{n+1}$ so that we can identify $\underline{P}_{n+1}=\underline{R}_{n+1} \oplus \underline{K}_{n+1}$.

Let us denote $\Pi:=P_{n+1, n}$. The isomorphism $\Pi_{!} \underline{P}_{n+1} \rightarrow \underline{P}_{n}$ induces an isomorphism $k$ : $\Pi_{\underline{1} \underline{K}_{n+1}} \rightarrow \underline{K}_{n}$ and a map $j: \Pi_{\underline{1}} \underline{R}_{n+1} \rightarrow \underline{R}_{n} \oplus \underline{K}_{n}$. Let $j_{R}: \Pi_{\underline{1}} \underline{R}_{n+1} \rightarrow \underline{R}_{n} ; j_{K}: \Pi_{\underline{1}} \underline{R}_{n+1} \rightarrow \underline{K}_{n}$ be its components. It follows that $j_{R}$ is the structure isomorphism of the inverse system $\underline{R}$. We need to change the splitting $\underline{P}_{n+1}=\underline{R}_{n+1} \oplus \underline{K}_{n+1}$ so as to get rid of $j_{K}$. Equivalently, we are to find a map $\varepsilon: \underline{R}_{n+1} \rightarrow \underline{K}_{n+1}$ so that

$$
k\left(\Pi_{!} \varepsilon\right)=j_{K}
$$

Let us use the conjugacy property:

$$
C: \operatorname{hom}\left(\Pi_{!} X ; Y\right) \stackrel{\sim}{\rightarrow} \operatorname{hom}\left(X ; \Pi^{-1} Y\right)
$$

for any $X \in \mathbf{L B A}_{n+1}^{\wedge}$ and $Y \in \mathbf{L B} \mathbf{A}_{n}^{\wedge}$. Let us apply $C$ to the equation (4):

$$
C(k) \varepsilon=C\left(j_{K}\right) \text {. }
$$


This equality means that $\varepsilon$ has to be a lifting of the following diagram

$$
\begin{gathered}
\underline{R}_{n+1} \\
\underline{K}_{n+1} \stackrel{C(k)}{\longrightarrow} \prod^{-1} \underline{K}_{n}
\end{gathered}
$$

The existence of such a lifting follows from the projectivity of $\underline{R}_{n+1}$ and surjectivity of the map $C(k)$, which follows from the isomorphism $\Pi_{\underline{1} \underline{K}_{n+1}} \rightarrow \underline{K}_{n}$.

4.3.3. Free systems. Given a positive integer $\langle k\rangle$ we have an object $\underline{S}^{k}$ in lim-LBA, $\lim -\underline{\mathbf{B A}}$ such that $\underline{S}_{n}^{k}:=h_{<k>}$ with the obvious isomorphisms $P_{n, n-1 !} h_{<k>}=h_{<k>}$.

Call an object $\underline{A}$ free if it is a direct sum of an admissible family of the form $\left\{h_{k_{i}}\right\}_{i \in I}$

It follows that such a family is admissible iff for every $N$ the set $\left\{i \mid k_{i}<N\right\}$ is finite. So, we will call a family $\left\{k_{i}\right\}_{i \in I}$ of positive integers admissible if it satisfies this condition. Given an admissible family of numbers $\mathbf{k}:=\left\{k_{i}\right\}_{i \in I}$ we denote by $\underline{S}^{\mathbf{k}}$ the corresponding free object.

LEMMA 4.6. Every object $\underline{P}$ in lim-BA, lim-LBA is a retraction of a free object. In other words, there is a free system $\underline{F}$ and an isomorphism $\underline{F}=\underline{P} \oplus \underline{K}$, where $\underline{K}$ is another projective system.

Proof. We will only prove it for lim- $\underline{\mathbf{L B A}}$, as the proof for lim- $\underline{\mathbf{B A}}$ is similar.

Because of Lemma 4.5, it suffices to find a free system that surjects onto $\underline{P}$. Such a free system can be constructed by induction as long as we establish the following fact:

Let $\underline{F}_{n} \in \mathbf{L B A} \wedge$ be a free finitely generated object

$$
\underline{F}_{n}=\bigoplus_{i=1}^{M} h_{<k_{i}>}
$$

Let $G \in \mathbf{L B} \mathbf{A}_{n+1}^{\wedge}$ be a free object generated by "the same elements" as $\underline{F}_{n}$ :

$$
G=\bigoplus_{i=1}^{M} h_{<k_{i}>},
$$

so that we have an isomorphism

$$
i: \Pi_{!} G=\underline{F}_{n},
$$

where $\Pi:=P_{n+1, n}$. Next, suppose we are given a surjection $q_{n}: \underline{F}_{n} \rightarrow \underline{P}_{n}$.

The statement is then as follows:

SUBLEMMA 4.7. There exist:

- a finitely generated free object $\Phi \in \mathbf{B A}_{n+1}$ which is a direct sum of finitely many copies of $h_{<n+1>\text {; }}$

- a surjection $q_{n+1}: G \oplus \Phi \rightarrow \underline{P}_{n+1}$ such that the map

$$
\Pi_{!}\left(q_{n+1}\right): \Pi_{!}(G \oplus \Phi) \rightarrow \Pi_{!} \underline{P}_{n+1}
$$

is equal to:

$$
\Pi_{!}(G \oplus \Phi)=\Pi_{!} G=\underline{F}_{n} \stackrel{p_{n}}{\rightarrow} \underline{P}_{n}=\Pi_{!} \underline{P}_{n+1} .
$$


It is clear that this sub-Lemma implies the Theorem, so let us prove the sub-Lemma. We have the following diagram

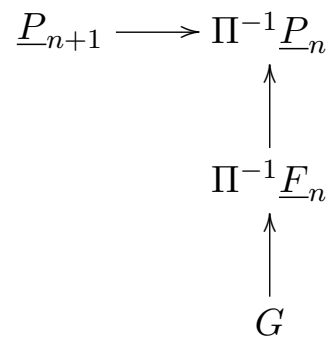

where all vertical arrows are surjective, therefore, we have a lifting $l: G \rightarrow \underline{P}_{n+1}$. Cokernel $C$ of this map is a surjective image of the kernel $K$ of the upper horizontal arrow $\underline{P}_{n+1} \rightarrow \Pi^{-1} \underline{P}_{n}=$ $\Pi^{-1} \Pi_{!} \underline{P}_{n+1}$. The target of this arrow is, by definition, the quotient of the source by its submodule generated by $\underline{P}_{n+1}(<n+1>)$. Therefore, this submodule, which is just $K$, is generated by elements in $K(<n+1>)$. Therefore, $C$, being a quotient of $K$, is also generated by $C(<n+1>)$, hence we have a surjection from a finite direct sum of a sufficiently large number of copies of $h_{<n+1>}$ to $C$. Let us denote the direct sum by $\Phi$ and the surjection by $\pi: \Phi \rightarrow C$. We have a lifting of $\pi$ to a map $\pi^{\prime}: \Phi \rightarrow \underline{P}_{n+1}$. It then follows that

$$
l \oplus \pi^{\prime}: G \oplus \Phi \rightarrow \underline{P}_{n+1}
$$

is a surjection. Let us set $q_{n+1}:=l \oplus \pi^{\prime}$. This surjection satisfies all the requirements.

4.3.4. Free systems form full SM-subcategories lim- $\underline{\mathbf{B A}}_{\text {free }} \subset \lim -\underline{\mathbf{B A}} ; \lim -\underline{\mathbf{L B A}} \underline{\mathbf{f r e e}}_{\text {fre }} \subset \lim \underline{\mathbf{L B A}}$. Let us describe the tensor product of free systems and the complex of homomorphisms of free systems. Our results imply that lim- $\underline{\mathbf{B A}}$ (resp. lim- $\underline{\mathbf{L B A}}$ ) is a Karoubian completion of lim- $\underline{\mathbf{B A}}_{\text {free }}$ (resp. lim- $\underline{\underline{\mathbf{L A}}}_{\text {free }}$ ).

Let $\mathbf{k}=\left\{k_{i}\right\}_{i \in I} ; \mathbf{l}=\left\{l_{j}\right\}_{j \in J}$. Let $\mathbf{k}+\mathbf{l}:=\left\{k_{i}+l_{j}\right\}_{(i, j) \in I \times J}$. It is clear the the family $\mathbf{k}+\mathbf{l}$ is admissible and that

$$
\underline{S}^{\mathbf{k}} \otimes \underline{S}^{\mathbf{l}} \cong \underline{S}^{\mathbf{k}+\mathbf{l}}
$$

Let us calculate

$$
\operatorname{hom}_{\lim -\underline{\mathbf{L B A}}}\left(\underline{S}^{\mathbf{k}^{1}} ; \underline{S}^{\mathbf{k}^{2}}\right)
$$

First of all, we see that

$$
\operatorname{hom}\left(\underline{S}_{k} ; \underline{S}_{l}\right) \cong \operatorname{liminv}_{n} \mathbf{L B A} \mathbf{A}_{n}(<k>;<l>) .
$$

Next, we have a natural projection

$$
\operatorname{hom}\left(\underline{S}^{\mathbf{k}^{1}} ; \underline{S}^{\mathbf{k}^{2}}\right) \rightarrow \prod_{i j} \operatorname{hom}\left(S_{k_{i}^{1}} ; S_{k_{j}^{2}}\right)
$$

One can check that

LEMMA 4.8. this map is an isomorphism.

Proof. Let us construct the inverse map.

We have natural projections

$$
P_{n}: \operatorname{hom}\left(S_{k_{i}^{1}} ; S_{k_{j}^{2}}\right) \rightarrow \mathbf{L B A}_{n}(<k>;<l>) .
$$


This projection vanishes as long as $k_{i}^{1}>n$ or $k_{j}^{2}>n$, therefore $P_{n}$ vanishes for almost all $i, j$, hence the maps $P_{n}$ extend to a map

$$
P_{n}: \prod_{i j} \operatorname{hom}\left(S_{k_{i}^{1}} ; S_{k_{j}^{2}}\right) \rightarrow \bigoplus_{i j} \mathbf{L B A}_{n}\left(<k_{i}^{1}>;<k_{j}^{2}>\right)=\mathbf{L B} \mathbf{A}_{n}\left(S_{n}^{\mathbf{k}^{1}} ; S_{n}^{\mathbf{k}^{2}}\right)
$$

It is immediate that, altogether, the maps $P_{n}$ define a map

$$
\prod_{i j} \operatorname{hom}\left(S_{k_{i}^{1}} ; S_{k_{j}^{2}}\right) \rightarrow \operatorname{hom}\left(S^{\mathbf{k}^{1}} ; S^{\mathbf{k}^{2}}\right) .
$$

Same result is true for hom $\operatorname{lim-\mathbf {BA}}\left(\underline{S}^{\mathbf{k}} ; \underline{S}^{\mathbf{l}}\right)$.

4.4. Conilpotent bialgebras as $\mathbf{S M}$ functors $\lim -\underline{B A}, \lim -\mathbf{L B A} \rightarrow \mathcal{C}$. Let $\mathcal{C}$ be a category satisfying the conditions from Sec. 4.1. Call a SM-functor $F: \lim$-LBA $\rightarrow \mathcal{C}$ admissible if it takes admissible direct sums in lim-LBA to direct sums in $\mathcal{C}$. More precisely, given an admissible family $\left\{\underline{A}^{i}\right\}_{i \in I}$ in lim- $\underline{\mathbf{L B A}}$, the natural map

$$
\bigoplus_{i \in I} F\left(\underline{A}^{i}\right) \rightarrow F\left(\bigoplus_{i \in I} \underline{A}^{i}\right)
$$

must be an isomorphism.

The notion of an admissible functor $\lim -\underline{\mathbf{B A}} \rightarrow \mathcal{C}$ is defined in the same way.

Let $\mathbf{L}$ be either lim-LBA or lim- $\underline{\mathbf{B A}}$. Admissible functors are uniquely determined by their restriction onto the full SM subcategory $\mathbf{L}_{h}$ of $\mathbf{L}$ formed by the objects $S^{<k>}$. However, not every $\mathrm{SM}$ functor $\mathbf{L}_{h} \rightarrow \mathcal{C}$ extends to $\mathbf{L}$.

On the other hand, given an admissible functor $F: \mathbf{L}_{\text {free }} \rightarrow \mathcal{C}$, it uniquely extends to an admissible functor $\mathbf{L} \rightarrow \mathcal{C}$.

Given an admissible functor $F: \lim \underline{\mathbf{L B A}} \rightarrow \mathcal{C}$ (or, equivalently, $F: \lim \underline{\mathbf{L B A}}_{\text {free }} \rightarrow \mathcal{C}$, the object $F\left(h_{<1>}\right)$ is naturally a conilpotent Lie bialgebra in $\mathcal{C}$. Indeed, $h_{<1>}$ is a Lie bialgebra in lim-LBA; the family $\left\{\mathbf{C O L I E}^{*}(<k>;<l>) \otimes h_{<1>}^{\otimes l}\right\}_{l=1,2, \ldots}$ is admissible, and we have a map

$$
\delta_{k} \in \operatorname{hom}_{\text {lim-LBA }}\left(h_{<1>}^{\otimes k} ; \bigoplus_{l=1}^{\infty} \operatorname{COLIE}^{*}(<k>;<l>) \otimes h_{<1>}^{\otimes l}\right)
$$

whose natural projections onto

$$
\operatorname{hom}_{\lim -\underline{\mathbf{L B A}}}\left(h_{<1>}^{\otimes k} ; \mathbf{C O L I E}^{*}(<k>;<l>) \otimes \mathfrak{h}_{<1>}^{\otimes l}\right)
$$

are $\delta_{k l}$.

Since $F$ preserves direct sums, $F\left(h_{<1>}\right)$ is automatically a conilpotent Lie bialgebra.

Analogously, given an admissible functor $F: \lim -\mathbf{B A} \rightarrow \mathcal{C}, F\left(h_{<1>}\right)$ is a conilpotent BA-algebra.

It turns out that an admissible functor $F: \mathbf{L} \rightarrow \mathcal{C}$ can be recovered up-to a unique isomorphism from the (Lie)-bialgebra $F\left(h_{<1>}\right)$. This is done as follows. Let $\mathfrak{g}$ be a conilpotent Lie bialgebra in $\mathcal{C}$. Let us construct an admissible functor $\mathcal{G}:=F_{\mathfrak{g}}: \lim \underline{\underline{\mathbf{L B A}}}_{\text {free }} \rightarrow \mathcal{C}$.

Given a free object $\underline{S}^{\mathbf{k}}$ set

$$
\mathcal{G}\left(\underline{S}^{\mathbf{k}}\right):=\bigoplus_{i} \mathfrak{g}^{\otimes k_{i}}
$$

Let us now construct a map

$$
\operatorname{hom}_{\lim -\underline{L B A}}\left(\underline{S}^{\mathbf{k}} ; \underline{S}^{\mathbf{l}}\right) \rightarrow \operatorname{hom}_{\mathcal{C}}\left(\mathcal{G}\left(\underline{S}^{\mathbf{k}}\right) ; \mathcal{G}\left(\underline{S}^{\mathbf{l}}\right)\right) .
$$


The LHS is identified with

$$
\prod_{i j} \operatorname{liminv}_{n} \mathbf{L B A}_{n}\left(<k_{i}>;<l_{j}>\right)=\prod_{i j n} \mathbf{C O L I E}\left(<k_{i}>;<n>\right) \otimes_{S_{n}} \mathbf{L I E}\left(<n>;<l_{j}>\right) .
$$

Let us now work with the RHS. The precomposition with $\delta_{k}$ gives rise to a map

$$
\prod_{i} \operatorname{hom}\left(\bigoplus_{n}\left(\mathbf{C O L I E}\left(<k_{i}>;<n>\right)^{*} \otimes \mathfrak{g}^{\otimes n}\right)^{S_{n}} ; \mathcal{G}\left(\underline{S}^{\mathbf{l}}\right)\right) \rightarrow \operatorname{hom}\left(\mathcal{G}\left(\underline{S}^{\mathbf{k}}\right) ; \mathcal{G}\left(\underline{S}^{\mathbf{l}}\right)\right) .
$$

The LHS is isomorphic to

$$
\prod_{n, i} \operatorname{COLIE}\left(<k_{i}>;<n>\right) \otimes_{S_{n}} \operatorname{hom}\left(\mathfrak{g}^{\otimes n} ; \bigoplus_{j} \mathfrak{g}^{\otimes l_{j}}\right)
$$

Next we have natural maps

$$
\mathbf{L I E}\left(<n>;<l_{j}>\right) \rightarrow \operatorname{hom}\left(\mathfrak{g}^{\otimes n} ; \mathfrak{g}^{\otimes l_{j}}\right) .
$$

Since $\mathbf{L I E}(<n>;<l>)=0$ for all $l>n$, we actually have a map

$$
\begin{aligned}
\prod_{n, i, j} \operatorname{COLIE}\left(<k_{i}>;<n>\right) \otimes_{S_{n}} \operatorname{LIE}( & \left.<n>;<l_{j}>\right) \\
& \rightarrow \prod_{n, i} \operatorname{COLIE}\left(<k_{i}>;<n>\right) \otimes_{S_{n}} \operatorname{hom}\left(\mathfrak{g}^{\otimes n} ; \bigoplus_{j} \mathfrak{g}^{\otimes l_{j}}\right) .
\end{aligned}
$$

This results in a map

$$
\prod_{n, i, j} \operatorname{COLIE}\left(<k_{i}>;<n>\right) \otimes_{S_{n}} \mathbf{L I E}\left(<n>;<l_{j}>\right) \rightarrow \operatorname{hom}\left(\mathcal{G}\left(\underline{S}^{\mathbf{k}}\right) ; \mathcal{G}\left(\underline{S}^{\mathbf{l}}\right)\right)
$$

Note that the LHS is identified with $\operatorname{hom}\left(\underline{S}^{\mathbf{k}} ; \underline{S}^{\mathbf{l}}\right)$. Thus, we have constructed a map

$$
\operatorname{hom}\left(\underline{S}^{\mathbf{k}} ; \underline{S}^{\mathbf{l}}\right) \rightarrow \operatorname{hom}\left(\mathcal{G}\left(\underline{S}^{\mathbf{k}}\right) ; \mathcal{G}\left(\underline{S}^{\mathbf{l}}\right)\right)
$$

One can easily check that this way $\mathcal{G}$ is an admissible functor lim- $\underline{\mathbf{L B A}}_{\text {free }} \rightarrow \mathcal{C}$. As $\lim -\underline{\mathbf{L B A}}$ is a Karoubian closure of lim- $\underline{\mathbf{L B A}}_{\text {free }}$, we have a canonical extension of $\mathcal{G}$ :

$$
F_{\mathfrak{g}}: \lim -\underline{\mathbf{L B A}} \rightarrow \mathcal{C}
$$

which is also admissible.

Analogously, given a conilpotent $\mathbf{B A}$-algebra $\mathfrak{m}$ in $\mathcal{C}$, one constructs an admissible functor

$$
F_{\mathfrak{m}}: \lim \underline{\underline{B A}} \rightarrow \mathcal{C}
$$

One can easily check that we have constructed mutually inverse equivalences between the category of conilpotent Lie bialgebras (resp. BA-algebras) in $\mathcal{C}$ and the category of admissible functors



Taking into account the SM-equivalence

$$
\mathbf{Q}: \lim -\underline{\mathbf{B A}} \rightarrow \lim \underline{\underline{\mathbf{L B A}}}
$$

which is compatible with admissible direct sums, we immediately get an equivalence between the categories of admissible functors $\lim -\underline{\mathbf{L B A}} \rightarrow \mathcal{C}$ and $\lim -\underline{\mathbf{B A}} \rightarrow \mathcal{C}$.

In particular, given a conilpotent Lie bialgebra $\mathfrak{g} \in \mathcal{C}$ we can convert it to an admissible functor $F_{\mathfrak{g}}: \lim \underline{\underline{\mathbf{L B A}}} \rightarrow \mathcal{C}$, then to a functor $F_{\mathfrak{g}}^{\prime}:=F_{\mathfrak{g}} \mathbf{Q}: \lim -\underline{\mathbf{B A}} \rightarrow \mathcal{C}$, and lastly to a conilpotent BA-algebra $\mathbf{Q}(\mathfrak{g}):=F_{\mathfrak{g}}^{\prime}\left(S^{<1>}\right)$. 
We have proven:

THEOREM 4.9. The functor $\mathbf{Q}$ is an equivalence of the category of conilpotent Lie bialgebras in $\mathcal{C}$ and conilpotent $\mathbf{B A}$-algebras in $\mathcal{C}$.

\section{Plan of the Proof of the Universal QUantization theorem 3.3}

We will link the projective systems $\underline{\mathbf{B A}}^{\wedge}$ and $\underline{\mathbf{L B A}}^{\wedge}$ by introducing a series of other projective systems of SMC and establishing a zigzag of equivalences of these projective systems. All these mediating projective systems will be constructed as cores with respect to $t$-structures [1] on some dg-categories.

Let us list the mediating projective systems we are going to introduce.

1) We replace the categories $\mathbf{B} \mathbf{A}_{n}^{\wedge}, \mathbf{L B} \mathbf{A}_{n}^{\wedge}$ with dg-categories $\mathbf{B} \mathbf{A}_{n}^{\text {proj }} ; \mathbf{L B} \mathbf{A}_{n}^{\text {proj }}$ which are just the categories of finite complexes of finitely generated projective objects in $\mathbf{B} \mathbf{A}_{n}^{\wedge} ; \mathbf{L B} \mathbf{A}_{n}^{\wedge}$; a precise definition is given in Appendix 1.

2) We will exploit the well known link between the bialgebras and brace-algebras. We will explain this link in detail in Section 5.1 Brace algebras are controlled by a certain dg-operad braces, hence we can construct a PROP BRACES generated by braces and obtain a projective system of SMC BRACES $_{n}$ which is obtained from BRACES by taking a quotient with respect to the ideal generated by $\mathrm{Id}_{<N>}$ for all $N>n$, in the same way as we obtained the projective systems $\underline{\mathbf{B A}}, \underline{\mathbf{L B A}}$. We will then establish SM weak equivalences (see Appendix 1 for the definition) between $\mathbf{B} \mathbf{A}_{n}^{\text {proj }}$ and $\mathbf{B R A C E S}_{n}^{\text {proj }}$ which will define a weak equivalence of the corresponding projective systems.

3) We will use a quasi-isomorphism of operads hoger $\rightarrow$ braces. We can construct a projective system of SMC HOGER in the same way as above: by taking a quotient by the ideal generated by $\operatorname{Id}_{<N>}, N>n$. We then automatically have weak equivalences HOGER $\rightarrow$ BRACES; $\underline{\text { HOGER }}^{\text {proj }} \rightarrow \underline{\text { BRACES }}^{\text {proj }}$.

4) We will use standard functors which convert Lie bialgebras into Gerstenhaber algebras and vise versa in order to construct weak equivalences (in both directions) between the projective systems $\underline{\mathbf{L B A}}^{\text {proj }}$ and $\underline{\mathbf{H O G E R}}^{\text {proj. }}$. This way we get a chain of weak equivalences linking $\underline{\mathbf{B A}}^{\text {proj }}$ and $\underline{\text { LBA }}^{\text {proj }}$.

5) We need to get back to projective systems of SM abelian categories $\underline{\mathbf{B A}}^{\wedge}, \underline{\mathbf{L B A}}^{\wedge}$.


structures and show that the weak equivalences between these categories that we have constructed are actually exact $t$-functors. One also easily checks that the tensor product and the projections $\mathbf{B A}_{n}^{\text {proj }} \rightarrow \mathbf{B A}_{n-1}^{\text {proj }}, \mathbf{L B A}_{n}^{\text {proj }} \rightarrow$ LBA $_{n-1}^{\text {proj }}$, BRACES $_{n}^{\text {proj }} \rightarrow$ BRACES $_{n-1}^{\text {proj }}$, etc. are right exact with respect to the $t$-structure.

We also check that the cores of $\mathbf{B} \mathbf{A}_{n}^{\text {proj }}, \mathbf{L B} \mathbf{A}_{n}^{\text {proj }}$ are equivalent to $\mathbf{B} \mathbf{A}_{n}^{\wedge} ; \mathbf{L B} \mathbf{A}_{n}^{\wedge}$.

We thus obtain induced equivalences of the cores and, thereby, the quantization theorem.

5.1. Bialgebras and brace algebras. The notion of brace algebra is defined in [9] see also [8]. We will reproduce the definition using a slightly different language.

5.1.1. Category of complexes. Let $\mathcal{C}$ be a $\mathrm{dg} \mathrm{SMC}$-category closed under finite direct sums. We can form SM categories $\mathbf{C o m}-\mathcal{C}$ (resp. $\mathbf{C o m}_{+} \mathcal{C}$ ) of complexes in $\mathcal{C}$ bounded from above (resp. the category of complexes bounded from below) in the following slightly non-standard way. Objects of $\mathbf{C o m}_{-} \mathcal{C}\left(\operatorname{resp} . \mathbf{C o m}_{+} \mathcal{C}\right)$ are sequences of objects $X^{n} \in \mathcal{C}$; and elements $d_{n m} \in Z^{1} \operatorname{hom}\left(X_{n}, X_{m}\right)$, $m>n$ such that 
1) for all $n<m$ :

$$
d\left(d_{n m}\right)+\sum_{n<k<m} d_{k m} d_{n k}=0
$$

note that $d$ is the differential in the complex $\operatorname{hom}_{\mathcal{C}}\left(X_{n} ; X_{m}\right)$

2) $X_{n}=0$ for all $n>>0$ (resp. $n<<0$ ).

Define the complex of homomorphisms.

For each $n$, let $H^{N}:=\prod_{k} \operatorname{hom}\left(X^{k}, Y^{N+k}\right)$. The differentials $d_{m n}$ on $X, Y$ give rise to maps

$$
D_{M N}: H^{M} \rightarrow H^{N}
$$

for all $N>M$. The elements $D_{M N}$ satisfy (6).

Let

$$
Z:=\operatorname{limdir}_{M} \prod_{N \geq M} H^{N}
$$

the sum of all $D_{M N}$ is a well defined map $D: Z \rightarrow Z$ and we assign

$$
\operatorname{hom}\left(X^{\bullet}, Y^{\bullet}\right):=\left(Z, d_{Z}+D\right) .
$$

The tensor product in both $\mathbf{C o m}_{+} \mathcal{C}, \mathbf{C o m}_{-} \mathcal{C}$ is given by the formula

$$
(X \otimes Y)^{n}=\bigoplus_{m} X^{m} \otimes Y^{n-m},
$$

where the direct sum is actually finite, and the differentials on $X \otimes Y$ are defined by the formula:

$$
d_{n m}=\sum_{i} d_{n-i, m-i}^{X} \otimes \operatorname{Id}_{Y_{i}} \pm \operatorname{Id}_{X_{i}} \otimes d_{n-i, m-i}^{Y}
$$

Thus, $\mathbf{C o m}_{-} \mathcal{C}, \mathbf{C o m}_{+} \mathcal{C}$ are dg SMC.

5.1.2. Definition of brace algebras. Let $V \in \mathcal{C}$. Let $W^{-k}:=T^{k} V, k=0,1,2, \ldots$; let $W^{\geq 0}=0$.

We define a brace structure on $V$ as

1) a collection of maps $d_{-n,-m}: W^{-n} \rightarrow W^{-m},-n<-m$ so that $\left(W^{\bullet},\left\{d_{n m}\right\}\right)$ is a complex in Com_C . Denote this complex by $\mathfrak{H}(V)$.

We have the standard coproduct $\Delta \in \operatorname{hom}_{\mathbf{C o m}_{-} \mathcal{C}}(\mathfrak{H}(V) ; \mathfrak{H}(V) \otimes \mathfrak{H}(V))$

given by the fromula

where

$$
\left.\Delta\right|_{V \otimes n}=\sum_{k=0}^{n} \Delta_{k}
$$

$$
\Delta_{k}: V^{\otimes n} \stackrel{\sim}{\rightarrow}(V)^{\otimes k} \otimes(V)^{\otimes n-k} \rightarrow \mathfrak{H}(V) \otimes \mathfrak{H}(V) .
$$

We demand that $\Delta$ must be compatible with the differential

2) an associative bialgebra structure on the complex $T V$ such that:

- the coproduct is as defined above, and

- The unit and counit are the standard ones:

$$
\mathbf{k} \rightarrow(V[1])^{\otimes 0} \rightarrow \mathfrak{H}(V) \rightarrow(V[1])^{\otimes 0}=\mathbf{k} .
$$

It is assumed that all the structure maps (the product, the co-product, the unit, the counit) are compatible with the differential; 
3) Let $p: \mathfrak{H}(V) \rightarrow V[1]$ be the natural projection and $m_{k, l}$ be the following components of the product:

$$
V[1]^{\otimes k} \otimes V[1]^{\otimes l} \rightarrow \mathfrak{H}(V) \otimes \mathfrak{H}(V) \stackrel{m}{\rightarrow} \mathfrak{H}(V) \stackrel{p}{\rightarrow} V[1]
$$

we then demand that $m_{k, l}=0$ for all $k \neq 1$. The components $m_{1, n}: V[1] \otimes V[1]^{\otimes n} \rightarrow V[1]$ are called the $n$-brace operations.

Note that in order to specify a brace algebra one has to prescribe the $n$-braces and also to prescribe the components of the differential

$$
M_{n}:(V[1])^{\otimes n} \rightarrow \mathfrak{H}(V) \stackrel{d}{\rightarrow} \mathfrak{H}(V) \rightarrow V[1]
$$

for all $n>1$ (the component $M_{1}$ is determined by the pre-existing differential on $V[1]$ ).

The operations $M_{n}$ are called higher cup-products.

This description implies that there exists a dg-operad controlling the structure of a brace algebra. We denote this operad by braces

We then have a tautological statement that $V \mapsto \mathfrak{H}(V)$ is a functor from the category of brace algebras in $\mathcal{C}$ to the category of $\mathbf{B A}$-algebras in $\mathbf{C o m}_{-} \mathcal{C}$.

5.1.3. Let $\mathbf{M}$ be a (non-symmetric) dg monoidal category enriched over a SMC $\mathcal{C}$ and let $A$ be a unital associative monoid in $\mathcal{C}$.

We then have the Hochschild complex $C^{\bullet}(A, A) \in \mathbf{C o m}_{+} \mathcal{C}$ defined in the standard way.

Assume that the unital map $\mathbf{1} \rightarrow A$ splits so that $A=\mathbf{1} \oplus \mathfrak{m}$. We then have a notion of the reduced Hochschild complex of $A, \bar{C}^{\bullet}(A, A)$.

It is well known that both $C^{\bullet}(A, A)$ and $\bar{C}^{\bullet}(A, A)$ are brace algebras in $\mathbf{C o m}_{+} \mathcal{C}$, see [9], [8].

5.2. Constructing a brace algebra out of a BA-algebra. In order to produce a natural definition we need a little bit of a categorical nonsense

5.2.1. Let $\mathcal{C}^{-}$be the category of all $\mathrm{dg}$ functors $\mathcal{C}^{\mathrm{op}} \rightarrow$ complexes. We have a SM-structure on $\mathcal{C}^{-}$defined in the same way as it is defined on $\mathcal{C}^{\wedge \mathrm{dg}}$ in Appendix 1.

The category $\mathcal{C}$ is naturally enriched over $\mathcal{C}^{-}$: given $X, Y \in \mathcal{C}$, define $\underline{\operatorname{Hom}}(X, Y) \in \mathcal{C}^{-}$by setting

$$
\underline{\operatorname{Hom}}(X, Y)(U):=\operatorname{hom}_{\mathcal{C}}(X \otimes U, Y)
$$

for all $U \in \mathcal{C}$.

5.2.2. Let $\mathfrak{m} \in \mathcal{C}$ be a BA-algebra. Let $H:=\mathbf{1} \oplus \mathfrak{m}$ so that $\mathcal{H}$ is an associative bialgebra. In particular, $H$ is an associative algebra in $\mathcal{C}$, and we can consider the category $\mathbf{M}$ of left $H$-modules in $\mathcal{C}$. It is a dg-category enriched over $\mathcal{C}^{-}$.

The coproduct on $H$ makes $\mathbf{M}$ into a monoidal category : given $X, Y \in \mathbf{M}$, their $\mathcal{C}$-tensor product has a natural left $H$-module structure. The counit map in $H$ endows the object $\mathbf{1}$ with the left $H$-action, we denote the corresponding object in $\mathbf{M}$ by $\mathbf{1}^{\prime}$.

Let $H^{\prime} \in \mathbf{M}$ be $H$ viewed as a left $H$-module. The coproduct on $H$ makes $H^{\prime}$ into a co-associative co-unital co-monoid in $\mathbf{M}$, hence $H^{\prime}$ is an associative unital monoid in $\mathbf{M}^{\mathrm{op}}$. The unit map produces an $H$-module splitting

$$
H^{\prime}=\mathbf{1}^{\prime} \oplus \mathfrak{m}^{\prime},
$$

where $\mathfrak{m}^{\prime}$ is $\mathfrak{m}$ viewed as a left $H$-module.

Consider the reduced Hochschild complex $\bar{C}^{\bullet}\left(H^{\prime} ; H^{\prime}\right)$; it is automatically a brace algebra in $\mathcal{C}^{-}$. Denote this algebra by $\mathfrak{B}(\mathfrak{m})$. Let us give a concrete description of $\mathfrak{B}(\mathfrak{m})$. 
We have

$$
\bar{C}^{n}\left(H^{\prime}: H^{\prime}\right)=\operatorname{hom}_{\mathbf{M}}\left(H^{\prime} ;\left(\mathfrak{m}^{\prime}\right)^{\otimes n}\right)
$$

It is clear that $H^{\prime}$ is a free $H$-module. In other words, for every $T \in \mathbf{M}$, $\operatorname{hom}_{\mathbf{M}}\left(H^{\prime} ; T\right)=h_{T}$, where the RHS is an object of $C^{-}$which represents $T$ viewed as an object of $\mathcal{C}$.

Therefore, we have an isomorphism

$$
\bar{C}^{n}\left(H^{\prime} ; H^{\prime}\right)=h_{\mathfrak{m} \otimes n}
$$

We can identify $\mathcal{C}$ with the full subcategory of $\mathcal{C}^{-}$consisting of representable functors, we then see that $\bar{C}^{\bullet}\left(H^{\prime}, H^{\prime}\right)$ is a complex from $\mathbf{C o m}_{+} \mathcal{C}$. As mentioned above, it is naturally a brace algebra. Let us denote this brace algebra by $\mathfrak{B}(\mathfrak{m})$ and give its explicit description.

1) As a complex, $\mathfrak{B}(\mathfrak{m})$ looks as follows:

$$
0 \rightarrow \mathfrak{m} \rightarrow \mathfrak{m}^{\otimes 2} \rightarrow \mathfrak{m}^{\otimes 3} \rightarrow \cdots,
$$

where $\mathfrak{m}$ is in the first degree and the differential is the co-bar differential induced by the coproduct on $\mathfrak{m}$.

The cup-products $M_{n}=0$ for $n>2$ and $M_{2}$ is just a free associative product

The brace operations are uniquely determined by demanding:

- the composition

$$
\mathfrak{m}[-1] \otimes \mathfrak{B}(\mathfrak{m})^{\otimes N} \rightarrow \mathfrak{B}(\mathfrak{m}) \otimes \mathfrak{B}(\mathfrak{m})^{\otimes N} \stackrel{m_{1 ; N}}{\rightarrow} \mathfrak{B}(\mathfrak{m})
$$

should vanish when $N>1$;

- the composition

$$
\mathfrak{m} \otimes(\mathfrak{m}[-1])^{\otimes n}[1] \stackrel{m_{1,1}}{\rightarrow} \mathfrak{B}(\mathfrak{m})[1] \otimes \mathfrak{B}(\mathfrak{m})[1] \rightarrow \mathfrak{B}(\mathfrak{m})[1]
$$

should be equal to:

$$
\mathfrak{m} \otimes(\mathfrak{m}[-1])^{\otimes n}[1] \stackrel{\Delta^{n}}{\longrightarrow} H^{\otimes n} \otimes(\mathfrak{m}[-1])^{\otimes n}[1] \stackrel{\mu^{\otimes n}}{\rightarrow}(\mathfrak{m}[-1])^{\otimes n}[1] \rightarrow \mathfrak{B}(\mathfrak{m})[1],
$$

where $H$ is the Hopf algebra $\mathbf{1} \oplus \mathfrak{m} ; \Delta^{n}: H \rightarrow H^{\otimes n}$ is the $n$-fold coproduct on $H$ and $\mu: H \otimes \mathfrak{m} \rightarrow \mathfrak{m}$ is the map induced by the product on $H$.

5.2.3. Let $\mathfrak{m}$ be a BA-algebra in $\mathcal{C}$ satisfying the following condition: there exists an $N>0$ such that $\mathfrak{m}^{\otimes N}=0$. No matter how exotic this condition is it will be the case in our situation, where $\mathfrak{m}$ will be the BA-algebra $h_{<1>} \in \mathbf{B A}_{n}$, so that $\left.N=n+1\right)$.

Provided this condition is the case, $\mathfrak{B}(\mathfrak{m})$ is just a finite complex in $\mathcal{C}$. Let us denote by $\operatorname{Com}(\mathcal{C})$ the $\mathrm{SMC}$ of finite complexes in $\mathcal{C}$ so that $\mathfrak{B}(\mathfrak{m})$ is a brace algebra in $\operatorname{Com}(\mathcal{C})$. Since $\mathfrak{B}(\mathfrak{m})$ is composed of non-zero powers of $\mathfrak{m}$, it follows that the $N$-th tensor power of $\mathfrak{B}(\mathfrak{m})$ is zero, therefore, $\mathfrak{H}(\mathfrak{B}(\mathfrak{m}))$ is a finite complex of finite complexes in $\mathcal{C}$ so that we can take the total complex of this double-complex obtaining this way a BA-algebra $\mathfrak{H}(\mathfrak{B}(\mathfrak{m}))$ in $\operatorname{Com}(\mathcal{C})$.

It turns out that in this case we have a natural map of BA-algebras $\alpha: \mathfrak{m} \rightarrow \mathfrak{H}(\mathfrak{B}(\mathfrak{m}))$ defined as follows:

as $\alpha$ must be compatible with the co-product it suffices to define its restriction

$$
\alpha_{r}: \mathfrak{m} \rightarrow(\mathfrak{B m})[1]
$$

we define it to be the identical map

$$
\mathfrak{m} \stackrel{\mathrm{Id}}{\rightarrow} \mathfrak{m} \subset(\mathfrak{B m})[1]
$$


The map $\alpha_{r}$ extends uniquely to a map $\alpha$ of coalgebras. It only remains to show it respects the product and the differential, which is straightforward.

5.3. Gerstenhaber algebras and Lie bialgebras. Throughout this section we assume that $\mathcal{C}$ is closed under kernels of projectors.

5.3.1. Let now $A$ be a Gerstenhaber algebra in $\mathcal{C}^{-}$. We can construct Harrison's complex of $A$, $\operatorname{Harr}(A) \in \mathbf{C o m}_{-} \mathcal{C}$. It is of the form

$$
\cdots \operatorname{Harr}(A)^{-n} \rightarrow \operatorname{Harr}(A)^{-n+1} \rightarrow \cdots \operatorname{Harr}(A)^{-1} \rightarrow 0 .
$$

where $\operatorname{Harr}(A)^{-n}[n]:=\left(\operatorname{lie}(n)^{*} \otimes k[1]^{\otimes n}\right) \otimes_{S_{n}} A^{\otimes n}$, and the differential is induced by the product on $A$ in the standard way. We have a cofree Lie algebra structure on $\operatorname{Harr}(A)$, and the differential is uniquely determined by the condition that it should be compatible with the Lie co-bracket and that the differential

$$
S^{2}(A)=\operatorname{Harr}(A)^{-2} \rightarrow \operatorname{Harr}(A)^{-1}=A
$$

is just the commutative product on $A$.

The Gerstenhaber bracket $\{$,$\} on A$ naturally extends to $\operatorname{Harr}(A)$ : let us define a map

$$
\text { [, ] : } \operatorname{Harr}(A)^{\otimes 2} \rightarrow \operatorname{Harr}(A)
$$

by demanding that:

1)

$$
\delta([x, y])=[\delta(x), y]+[x, \delta(y)]
$$

2) The co-restriction

$$
\operatorname{Harr}(A) \otimes \operatorname{Harr}(A) \stackrel{[,]}{\longrightarrow} \operatorname{Harr}(A) \stackrel{r}{\rightarrow} A[1]
$$

should coincide with the map

$$
\operatorname{Harr}(A) \otimes \operatorname{Harr}(A) \stackrel{r \otimes r}{\longrightarrow} A[1] \otimes A[1]=A \otimes A[2] \stackrel{\{,\}}{\longrightarrow} A[-1][2]=A[1],
$$

where $\{$,$\} is the bracket on the Gerstenhaber algebra A$.

One can easily check that there is a unique map [,] satisfying 1$), 2)$ and that $(\operatorname{Harr}(A) ; d ;[,] ; \delta)$ is a dg Lie bialgebra. We denote this Lie bialgebra by $\mathfrak{L}(A)$.

5.3.2. Let us construct the inverse map. Let $\mathfrak{g}$ be a Lie bialgebra in $C$. Consider its ChevalleyEilenberg cochain complex $C^{\bullet}(\mathfrak{g})$ with respect to the cobracket. This complex is an object of $\operatorname{Com}_{+} \mathcal{C}$; we have $C^{n}(\mathfrak{g})=\Lambda^{n}(\mathfrak{g})$ and the differential is defined by the cobracket $\delta$ on $\mathfrak{g}$. It is well known that $C^{\bullet}(\mathfrak{g})$ is a commutative algebra in $\mathbf{C o m}_{+} \mathcal{C}$.

We can define the Gerstenhaber bracket

$$
\{,\}: C^{\bullet}(\mathfrak{g})^{\otimes 2} \rightarrow C^{\bullet}(\mathfrak{g})[1]
$$

by asserting that

1) the bracket should obey the Leibnitz rule;

2) the restriction

$$
\mathfrak{g}[-1]^{\otimes 2} \rightarrow C^{\bullet}(\mathfrak{g})^{\otimes 2} \stackrel{\{,\}}{\longrightarrow} C^{\bullet}(\mathfrak{g})[-1]
$$

should coincide with the map

$$
\mathfrak{g}[-1]^{\otimes 2} \stackrel{[,]}{\rightarrow} \mathfrak{g}[-1][-1] \rightarrow C^{\bullet}(\mathfrak{g})[-1] .
$$


It is easy to check that this way, $C^{\bullet}(\mathfrak{g})$ is a Gerstenhaber algebra in $\mathbf{C o m}_{+} \mathcal{C}$. Denote this Gerstenhaber algebra by $\mathfrak{G}(\mathfrak{g})$

5.3.3. Given a Lie bialgebra $\mathfrak{g}$ in $C$ satisfying $\mathfrak{g}^{\otimes N}=0$ for $N$ large enough, define a map

$$
\mathfrak{g} \rightarrow \mathfrak{L} \mathfrak{G}(\mathfrak{g}),
$$

where the RHS is viewed as a finite complex in $\mathcal{C}$.

As the RHS in (7) is cofree as a Lie coalgebra, it suffices to define the composition

$$
\mathfrak{g} \rightarrow \mathfrak{L} \mathfrak{G}(\mathfrak{g}) \rightarrow \mathfrak{G}(\mathfrak{g})[1]
$$

We set this composition to be the obvious inclusion

$$
i: \mathfrak{g} \rightarrow \mathfrak{G}(\mathfrak{g})[1]=C^{\bullet}(\mathfrak{g})[1]
$$

onto the term $C^{1}(\mathfrak{g})=\mathfrak{g}$.

One can easily check that this way we indeed get a Lie bialgebra map. Note that the map $i$ can be extended to a map of Lie bialgebras beacause the cobracket on $\mathfrak{g}$ is conilpotent.

5.3.4. Let $A$ be a Gerstenhaber algebra in $\mathcal{C}$ satisfying $A^{\otimes N}=0$ for $N$ large enough.

Let us construct a map of Gerstenhaber algebras

$$
\mathfrak{G} \mathfrak{L}(A) \rightarrow A,
$$

where the LHS is viewed as a finite complex in $\mathcal{C}$. As the LHS in (8) is free, it suffices to prescribe the restriction of this map onto the generators:

$$
\mathfrak{L}(A)[-1]=\operatorname{Harr}(A)[-1] \rightarrow A
$$

which we set to be the obvious projection onto $\operatorname{Harr}^{-1}(A)=A$.

It is easy to check that this way we indeed get a map of Gerstenhaber algebras.

5.4. Bialgebras and brace algebras: translation into the universal language. We use freely the notations from the Appendix 1.

5.4.1. We have a universal brace algebra $<1>\in$ BRACES. The projections $P_{N}:$ BRACES $\rightarrow$ BRACES $_{N}$ endow

$$
P_{N}<1>=<1>\in \mathbf{B R A C E S}_{N}
$$

with a brace-algebra structure.

Let $h_{<1>} \in \mathbf{B R A C E S}_{N}^{\text {free }}$ be Ioneda's image of $<1>$

$$
h_{<1>}(<k>):=\operatorname{hom}_{\mathbf{B R A C E S}_{N}}(<k>;<1>) .
$$

We can apply the functor $\mathfrak{H}$ to the brace algebra $h_{<1>} \in \mathbf{B R A C E S}_{N}^{\text {free }}$ so that we get an object

$$
\mathfrak{H}_{N}:=\mathfrak{H}\left(h_{<1>}\right)
$$

which is actually a finite complex of objects in $\mathbf{B R A C E S} \mathbf{S}_{N}^{\text {free }}$, hence, this complex naturally defines

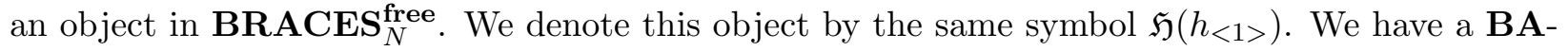
structure on $\mathfrak{H}\left(h_{<1>}\right)$.

This implies that we have a SM functor

$$
\mathfrak{H}_{N}^{\prime}: \mathbf{B A} \rightarrow \mathbf{B R A C E S}_{N}^{\text {free }}
$$

such that $\mathfrak{H}^{\prime}(<m>)=\left(\mathfrak{H}_{N}\right)^{\otimes m}$. 
One can easily check that $\mathfrak{H}_{N}^{\otimes m}=0$ for all $m>N$. This implies that we actually have a SM functor

$$
\mathfrak{H}_{N}: \mathbf{B A}_{N} \rightarrow \text { BRACES }_{N}^{\text {free }}
$$

As explained in Appendix 1, given any such a functor, we have its canonical SM extensions

$$
\begin{gathered}
\mathfrak{H}_{N}: \mathbf{B A}_{N}^{\text {free }} \rightarrow \text { BRACES }_{N}^{\text {free }} ; \\
\mathfrak{H}_{N}: \mathbf{B A}_{N}^{\text {proj }} \rightarrow \text { BRACES }_{N}^{\text {proj }} .
\end{gathered}
$$

It is easy to see that the SM functors $\mathfrak{H}_{N}$ are compatible with the projections

$$
\begin{gathered}
P_{N M}: \mathbf{B A}_{N} \rightarrow \mathbf{B A}_{M} ; \\
P_{N M}: \mathbf{B R A C E S}_{N} \rightarrow \text { BRACES }_{M}
\end{gathered}
$$

so that we get a map of projective systems

$$
\begin{aligned}
& \underline{\mathfrak{H}}: \underline{\mathbf{B A}}^{\text {free }} \rightarrow \underline{\text { BRACES }}^{\text {free }} ; \\
& \underline{\mathfrak{H}}: \underline{\text { BA }}^{\text {proj }} \rightarrow \underline{\text { BRACES }}^{\text {proj }} .
\end{aligned}
$$

5.4.2. Let us now construct a functor in the opposite direction

$$
\underline{\mathfrak{B}}: \underline{\mathrm{BRACES}}^{\text {free }} \rightarrow \underline{\mathrm{BA}}^{\text {free }}
$$

using as a prototype the construction from Sec. 5.1.2

We have BA-algebras $<1>\in \mathbf{B A}_{N}$ and $h_{<1>} \in \mathbf{B A}_{N}^{\text {free }}$. Therefore, we have a brace algebra $\mathfrak{B}\left(h_{<1>}\right)$ which is a finite complex of objects from $\mathbf{B} \mathbf{A}_{N}^{\text {free }}$, hence, it defines an object in $\mathbf{B} \mathbf{A}_{N}^{\text {free }}$ which is a brace algebra. We denote this brace algebra in $\mathbf{B} \mathbf{A}_{N}^{\text {free }}$ by $\mathfrak{B r}_{N}$.

We thus get SM functors

$$
\mathfrak{B}_{N}^{\prime}: \mathbf{B R A C E S} \rightarrow \mathbf{B A}_{N}^{\text {free }},
$$

where $\mathfrak{B}_{N}^{\prime}(<m>):=\left(\mathfrak{B r}_{N}\right)^{\otimes m}$.

One checks that $\mathfrak{B}_{N}^{\prime}(<m>)=0$ for all $m>N$ so that we automatically get functors

$$
\mathfrak{B}_{N}^{\prime}: \mathbf{B R A C E S}_{N} \rightarrow \mathbf{B A}_{N}^{\text {free }}
$$

which canonically extend to SM functors

$$
\begin{gathered}
\mathfrak{B}_{N}: \mathbf{B R A C E S}_{N}^{\text {free }} \rightarrow \mathbf{B A}_{N}^{\text {free }} \\
\mathfrak{B}_{N}: \mathbf{B R A C E S}_{N}^{\text {proj }} \rightarrow \mathbf{B A}_{N}^{\text {proj }} .
\end{gathered}
$$

These maps produce maps of systems

$$
\begin{aligned}
& \underline{\mathfrak{B}}: \underline{\mathrm{BRACES}}^{\text {free }} \rightarrow{\underline{\mathrm{BA}^{\text {free }}}}^{\text {froj }} \\
& \underline{\mathfrak{B}}: \underline{\mathrm{BRACES}}^{\text {proj }} \rightarrow \underline{\mathrm{BA}}^{\text {proj }}
\end{aligned}
$$

The formulas from 5.2.3 are applicable in our setting so that we have a SM natural transformation

$$
\alpha: \operatorname{Id} \rightarrow \underline{\mathfrak{B}} \underline{\mathfrak{H}} .
$$

LEMMA 5.1. This map is a quasi-isomorophism of SM-functors. 
Proof. It suffices to check that the maps

$$
\alpha_{N}\left(h_{<n>}\right): h_{<n>} \rightarrow \mathfrak{B}_{N} \mathfrak{H}_{N}\left(h_{<n>}\right)
$$

are quasi-isomorphisms in $\mathbf{B} \mathbf{A}_{N}^{\text {free }}$. Since all the functors involved are strong SM, it suffices to consider the case $n=1$ and to check that the map

$$
h_{<1>} \rightarrow \mathfrak{B}_{N} \mathfrak{H}_{N}\left(h_{<1>}\right)
$$

is a quasi-isomorphism.

We have

$$
\mathfrak{H}_{N}\left(h_{<1>}\right)=0 \rightarrow h_{<N>} \rightarrow h_{<N-1>} \rightarrow \cdots h_{<1>} \rightarrow 0
$$

where the differential is the bar differential induced by the associative algebra structure on $h_{<1>} \in \mathbf{B R A C E S}_{N}^{\text {free }}$ and the term $h_{<1>}$ is in the co-homological degree -1 .

Analogously,

$$
\mathfrak{B}_{N}\left(h_{<1>}\right)=\left(0 \rightarrow h_{<1>} \rightarrow h_{<2>} \rightarrow \cdots \rightarrow h_{<N>} \rightarrow 0\right)
$$

where the differential is just the co-bar differential induced by the co-associative coalgebra structure on $\mathfrak{h}_{<1>} \in \mathbf{B A}_{N}^{\text {proj }}$.

The composition $\mathfrak{B}_{N} \mathfrak{H}_{N} h_{<1>}$ is isomorphic to the bar construction of the co-bar construction applied to $h_{<1>} \in \mathfrak{H}_{N}^{\text {proj }}$ viewed as a co-associative co-algebra. Let us temporarily forget the differential and view $\mathfrak{B}_{N} \mathfrak{H}_{N} h_{<1>}$ as a functor from $\mathfrak{H}_{N}^{\text {op }}$ to the category of finite-dimensional graded vector spaces. We then have an isomorphism

$$
\mathfrak{B}_{N} \mathfrak{H}_{N} h_{<1>} \cong T\left(T\left(h_{<1>}[-1]\right)[1]\right),
$$

where $T X$ stands for $\bigoplus_{n=1}^{\infty} X$. Since $\left(h_{<1>}\right)^{\otimes>N}=0$, the RHS in (11) is a finite direct sum of tensor powers of $X$.

The RHS of (11) has an additional grading Gr by the number of tensor factors. Introduce a filtration

$$
F^{M} T\left(T\left(h_{<1>}[-1]\right)[1]\right):=\bigoplus_{K \geq M} \operatorname{Gr}^{K} T\left(T\left(h_{<1>}[-1]\right)[1]\right) .
$$

We see that the differential on $\mathfrak{B}_{N} \mathfrak{H}_{N} h_{<1>}$ preserves this filtration. Let us pass to the associated graded quotient of $\mathfrak{B}_{N} \mathfrak{H}_{N} h_{<1>}$ with respect to this filtration. The $M$-th graded piece of this quotient is isomorphic to

$$
\mathrm{Gr}^{M} T\left(T\left(h_{<1>}[-1]\right)[1]\right)
$$

with the bar differential coming from the free associative algebra structure on $T\left(h_{<1>}[-1]\right)$. It is well known that these complexes are acyclic for all $M>1$.

We also have $\operatorname{Gr}^{1} T\left(T\left(h_{<1>}[-1]\right)[1]\right)=h_{<1>}$. The Proposition now follows easily

5.4.3. The composition $\underline{\mathfrak{H} \mathfrak{B}}$ is a weak equivalence. We will prove

PROPOSITION 5.2. The compositions;

$$
\begin{aligned}
& \underline{\mathfrak{H}} \mathfrak{B}: \underline{\text { BRACES }}^{\text {free }} \rightarrow \underline{\text { BRACES }}^{\text {free }} ; \\
& \underline{\mathfrak{H}}_{\mathfrak{B}}: \underline{\text { BRACES }}^{\text {proj }} \rightarrow \underline{\text { BRACES }}^{\text {proj }}
\end{aligned}
$$

are weak equivalences. 
We denote $E:=\underline{\mathfrak{H}} \underline{\mathfrak{B}}$. The Proposition will follow from a series of Lemmas.

LEMMA 5.3. The object $E_{N}\left(h_{<1>}\right)$ is weakly equivalent to $h_{<1>} \in \mathbf{B R A C E S}_{N}^{\text {free }}$.

Proof. Similar to Lemma 5.1. The object $E_{N}\left(h_{<1>}\right)$ is isomorphic to the co-bar construction of the bar-construction applied to the homotopy associative algebra $h_{<1>} \in \mathbf{B R A C E S}_{N}^{\text {free }}$. Up-to the differential, we have an isomorphism

$$
E_{N}\left(h_{<1>}\right)=T\left(T\left(h_{<1>}[1]\right)[-1]\right)
$$

We then introduce the grading Gr by the number of the tensor factors and set

$$
F^{M} T\left(T\left(h_{<1>}[1]\right)[-1]\right):=\bigoplus_{K \leq M} \operatorname{Gr}^{K} T\left(T\left(h_{<1>}[1]\right)[-1] .\right.
$$

We then see that the differential preserves this filtration and pass to the associated graded quotients. These quotients are acyclic in all gradings greater than 1 and the quotient of grading 1 is isomorphic to $h_{<1>}$.

5.4.4. Let $X:=\mathfrak{B}_{N} \mathfrak{h}_{<1>}, N \geq 2$. We have a natural map

$$
\operatorname{BRACES}(<2>;<1>) \rightarrow \operatorname{hom}_{\mathbf{B A}_{N}^{\text {free }}}\left(X^{\otimes 2} ; X\right)
$$

LEMMA 5.4. This map is a quasi-isomorphism.

Proof. Let $j: \mathbf{A S S O C}_{N}=\mathbf{C O A S S}_{N}^{\text {op }} \rightarrow \mathbf{B A}_{N}^{\text {op }}$ be the natural inclusion of PROPs. We have $X=j_{!} Y$, where

$$
Y=0 \rightarrow h_{<1>} \rightarrow h_{<2>} \rightarrow h_{<3>} \rightarrow \cdots \rightarrow h_{<N>} \rightarrow 0
$$

is the cobar-complex of the coalgebra $h_{<1>}$, same as in (10).

Therefore, $X^{\otimes 2}=j_{!} Y^{\otimes 2}$ and, by the conjugacy property,

$$
\operatorname{hom}_{\mathbf{B A}_{N}^{\text {free }}}\left(X^{\otimes 2} ; X\right) \cong \operatorname{hom}_{\mathbf{C O A S S}_{N}^{\text {free }}}\left(Y^{\otimes 2} ; j^{-1} X\right)
$$

Decomposition (1) implies that

$$
j^{-1} h_{<m>} \cong \bigoplus_{r=m}^{N} \mathbf{A S S O C}(<r>;<m>) \otimes_{S_{r}} h_{<r>} .
$$

Next, we have

\section{SUBLEMMA $\mathbf{5 . 5}$.}

if $r \neq 2$;

$$
H^{\bullet}\left(\operatorname{hom}_{\mathbf{C O A S S}_{N}^{\text {free }}}\left(Y^{\otimes 2}, h_{<r>}\right)\right)=0
$$

$$
\operatorname{hom}\left(Y^{\otimes 2}, h_{<2>}\right) \cong \mathbf{C O A S S}(<2>;<2>)[2] \cong(\mathbf{k} \oplus \mathbf{k})[2] .
$$

Proof. Let $V_{r}$ be the $r$-dimensional vector space with the fixed basis $e_{1}, e_{2}, \ldots, e_{r}$; one can view it as a space with $r$ gradings so that the $i$-th grading of $e_{j}$ equals the Kronecker symbol $\delta_{i j}$. Given any vector space $U$ with $r$ gradings let $|U|$ be its homogeneous part whose all gradings are 1 .

Let $A_{r}:=T V_{r}$ be the free associative algebra generated by $V_{r}$. We then have

$$
h_{<r>}(<k>)=\mathbf{C O A S S}(<k>;<r>)=\operatorname{ASSOC}(<r>;<k>)=\left|A_{r}^{\otimes k}\right|,
$$

where $h_{<r>} \in \mathbf{C O A S S}_{N}^{\text {free }}$ is the functor represented by $<r>$.

The complex $\operatorname{hom}\left(Y^{\otimes 2} ; h_{<r>}\right)$ is isomorphic to

$$
\left|\left(\mathbb{B} A_{r}\right)^{\otimes 2}\right|,
$$


where $\mathbb{B} A_{r}$ is the standard bar complex of $A_{r}$. We have a natural quasi-isomorphism

$$
\mathbb{B} A_{r} \rightarrow V_{r}[1],
$$

hence a quasi-isomorphism

$$
\left|\left(\mathbb{B} A_{r}\right)^{\otimes 2}\right| \rightarrow\left|\left(V_{r}[1]\right)^{\otimes 2}\right|
$$

the complex on the RHS is 0 unless $r \neq 2$ in which case the complex on the RHS is a 2-dimensional space canonically identified with $\mathbf{C O A S S}(<2>;<2>)[2]$

This sublemma implies that the map

$$
\operatorname{ASSOC}(<2>;<n>) \otimes_{S_{2}} h_{<2>} \rightarrow j^{-1} h_{<n>}
$$

induces a quasi-isomorphism

$$
\begin{aligned}
\operatorname{ASSOC}(<2>;<n>)[2]= & \operatorname{hom}\left(Y^{\otimes 2} ; \operatorname{ASSOC}(<2>;<n>) \otimes_{S_{2}} \mathfrak{h}_{<2>}\right) \\
& \rightarrow \operatorname{hom}\left(Y^{\otimes 2} ; j^{-1} h_{<n>}\right)
\end{aligned}
$$

In particular, these spaces are homotopy equivalent to 0 if $n>2$.

Taking into account (13) and the sub-Lemma, we get a quasi-isomorphism

$$
\operatorname{hom}\left(Y^{\otimes 2} ; j^{-1} X\right) \rightarrow \operatorname{hom}\left(Y^{\otimes 2} ; \text { Cone }\left[h_{<1>} \rightarrow h_{<2>}\right]\right)[-1]
$$

The latter complex is quasi-isomorphic to the complex

$$
0 \rightarrow \operatorname{ASSOC}(<2>;<1>) \rightarrow \operatorname{ASSOC}(<2>;<2>) \rightarrow 0,
$$

where the term $\operatorname{ASSOC}(<2>;<2>)$ is in the cohomological degree 0 .

Let us now compute the trough map

$$
\begin{aligned}
H^{\bullet} \operatorname{braces}(2) \rightarrow H^{\bullet} \operatorname{hom}\left(X^{\otimes 2} ; X\right) & \\
& =H^{\bullet}\left(Y^{\otimes 2} ; j^{-1} X\right) \\
& \rightarrow H^{\bullet}[(\operatorname{ASSOC}(<2>;<1>) \rightarrow \operatorname{ASSOC}(<2>;<2>)]
\end{aligned}
$$

The cohomology $H^{\bullet} \operatorname{braces}(2)$ has 2 generators: $\cup \in H^{0}$ and $b \in H^{-1}$.

The cup product is represented by the map $X^{\otimes 2} \rightarrow X$ given by the free tensor product $h_{<k>}[-k] \otimes$ $h_{<l>}[-l] \rightarrow h_{<k+l>}[-k-l]$. This map produces the identity map in $\operatorname{ASSOC}(<2>;<2>)$.

The bracket $b$ is the anti-symmetrization of the brace operation

$$
X \otimes X \rightarrow X
$$

We only need to know its restriction onto $h_{<1>} \otimes h_{<1>}$ which is induced by the commutator

$$
m_{12}-m_{21}:<1>\otimes<1>\rightarrow<1>
$$

where $m_{12} \in \mathbf{A S S O C}(<2>;<1>)$ is the associative product.

We now see that the map (14) is an isomorphism. This proves the statement.

Let us consider the map of PROP's

$$
\operatorname{BRACES}_{N} \rightarrow \mathbf{F U L L}\left(E_{N}\left(h_{<1>}\right)\right)
$$

naturally induced by the functor $E_{n}$.

LEMMA 5.6. This map is a quasi-isomorphism of PROPs 
Proof. As was shown above, the object $E_{N}\left(h_{<1>}\right)$ is weakly equivalent to $h_{<1>}$. Therefore, the PROP FULL $\left(E_{N}\left(h_{<1>}\right)\right)$ is weakly equivalent to BRACES $\mathbf{B R}_{N}$. Therefore, we have an isomorphism

$$
H^{\bullet} \mathbf{F U L L}\left(E_{N}\left(h_{<1>}\right)\right) \rightarrow H^{\bullet} \mathbf{B R A C E S}
$$

As the operad braces is weakly equivalent to the operad ger, so are the PROPs generated by these operads and we have an isomorphism

\section{$H^{\bullet} \mathrm{BRACES} \cong \mathrm{GER}$.}

Thus we have a chain of maps

$$
\mathbf{G E R} \cong H^{\bullet} \mathbf{B R A C E S} \rightarrow H^{\bullet} \mathbf{F U L L}\left(E_{n}\left(h_{<1>}\right)\right) \cong \mathbf{G E R}
$$

and it suffices to prove that this through map is an isomorphism. Since the PROP GER is generated by its binary operations i.e. $\operatorname{hom}(<2>;<1>)$, it suffices to check that our map is an isomorphism when restricted onto the two-dimensional space hom $([2] ;[1])$.

we have a chain of maps

$$
\begin{aligned}
\operatorname{ger}(2) \stackrel{1}{\rightarrow} H^{\bullet} \operatorname{hom}_{\mathbf{B A}_{N}}\left(X^{\otimes 2} ; X\right) & \\
\stackrel{2}{\rightarrow} H^{\bullet} \mathbf{F U L L}\left(E_{N}\left(h_{<1>}\right)\right)(<2>;<1>) & \stackrel{3}{\rightarrow} H^{\bullet} \operatorname{hom}_{\mathbf{B A} \mathbf{A}_{N}}\left(\mathfrak{B H}(X)^{\otimes 2} ; \mathfrak{B H}(X)\right)
\end{aligned}
$$

The arrow 1 is induced by the map (12);

the arrow 2 is induced by the functor $\mathfrak{H}_{N}$ : note that we have an isomorphism $E_{N} h_{<1>} \cong \mathfrak{H}_{N}(X)$; the arrow 3 is induced by the functor $\mathfrak{B}_{N}$.

The arrow 1 is an isomorphism (Lemma 5.4); the composition of arrows 2,3 is an isomorphism because it is induced by the composition $\mathfrak{B}_{N} \mathfrak{H}_{N}$ which is a weak equivalence by Lemma 5.1. Taking into account that the dimensions of all spaces involved are equal and finite, we must conclude that all arrows in this sequence are isomorphisms, whence the statement.

\subsection{5. proof of the Proposition}

Let us prove that $E: \underline{\text { BRACES }}^{\text {free }} \rightarrow \underline{\text { BRACES }}^{\text {free }}$ is a weak equivalence. The statement for $\underline{\text { BRACES }}^{\text {proj }}$ will then follows automatically as $\mathbf{B R A C E S}_{n}^{\text {proj }}$ is the Karoubian closure of $\operatorname{BRACES}_{n}^{\text {free }}$.

Lemma 5.6 implies the following: given free finitely generated objects $F, G$ in $\mathbf{B R A C E S}_{n}^{\text {free }}$ (that is each of $F, G$ is a finite direct sum of the form $\bigoplus_{i} h_{<k_{i}>}\left[n_{i}\right]$ ), we have

$$
E_{n}: \operatorname{hom}_{\mathbf{B R A C E S}_{N}^{\text {free }}}(F, G) \rightarrow \operatorname{hom}_{\mathbf{B R A C E S}_{N}^{\text {free }}}\left(E_{N}(F), E_{N}(G)\right)
$$

is a quasi-isomorphism. The standard argument then implies that the same holds true if $F, G$ are finite complexes of finitely generated free objects, i.e. of $F, G$ are any objects in BRACES free ${ }_{N}$ It only remains to prove that $E_{N}$ is essentially surjective. Let us show by induction that every length $M$ complex of finitely generated free objects in $\mathbf{B R A C E S}{ }_{n}^{\text {free }}$ is quasi-isomorphic to an object from the image of $E_{n}$ Indeed, Lemma 5.3 implies that $h_{<k>}$ is quasi-isomorphic to $E_{n} h_{<k>}$; this readily implies that given any free finitely generated object $F \in \mathbf{B R A C E S}{ }^{\text {free }}, E_{N}(F)$ is quasi-isomorphic to $F$. This covers the case $M=1$.

The transition goes as follows. Every complex of length $M$ is isomorphic to a Cone $(f: K \rightarrow F)$, where $K$ is a complex of length $M-1$ and $F$ is free. The induction assumption implies that there is $K^{\prime} \in$ BRACES $^{\text {free }}$ and a quasi-isomorphism $k: E\left(K^{\prime}\right) \rightarrow K$. We also have a quasi-isomorphism 
$\phi: E(F) \rightarrow F$. This implies that there is an arrow $f^{\prime}: K^{\prime} \rightarrow F$ such that $\phi E_{N}\left(f^{\prime}\right)-f k=d \gamma$, where $\gamma \in \operatorname{hom}^{-1}\left(E_{N}\left(K^{\prime}\right) ; F\right)$. These data produce a quasi-isomorphism of $E_{N}\left(\operatorname{Cone}\left(f^{\prime}\right)\right)$ and Cone $(f)$.

5.4.6. Gerstenhaber algebras. We have quasi-isomorphisms of operads

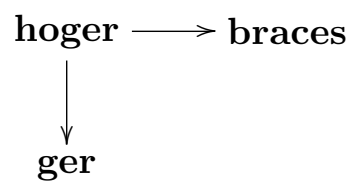

Whence induced equivalences of projective systems of PROPs



5.4.7. Lie bialgebras and Gerstenhaber algebras in the universal language. The constructions of 5.3 give rise to maps of the projective systems

$$
\underline{\text { LBA }}^{\text {proj }} \underset{\underline{\underline{G}}}{\stackrel{\underline{\mathfrak{L}}}{\rightleftarrows} \text { GER }^{\text {proj }}}
$$

We have natural transformations

$$
\mathrm{Id} \rightarrow \underline{\mathfrak{L} \mathfrak{G}} ; \mathrm{Id} \rightarrow \underline{\mathfrak{G} \mathfrak{L}}
$$

These natural transformations are weak equivalences. Hence so are $\underline{\mathfrak{L}}$ and $\underline{\mathfrak{G}}$.

Let us prove all these statements.

5.4.8. Constructing the maps $\underline{\mathfrak{L}}, \underline{\mathfrak{G}}$. The construction is similar to that in 5.4. The major difference is that, since our construction involve tensoring with non-regular representations of the symmetric group, the resulting objects will be projective, not necessarily free.

The functor $\underline{\mathfrak{L}}$ We have a universal Gerstenhaber algebra $<1>\epsilon \mathbf{G E R}$. Its images $<1>\epsilon$ $\mathbf{G E R}_{n} ; h_{<1>} \in \mathbf{G E R}_{n}^{\text {free }}$ are Gerstenhaber algebras. We can apply the functor $\mathfrak{L}$ so as to get Lie bialgebras

$$
\mathfrak{L}_{n}^{\prime}:=\mathfrak{L}\left(h_{<1>}\right) \in \mathbf{G E R}_{n}^{\text {proj }} .
$$

These bialgebras can be interpreted as SM functors

$$
\mathfrak{L}_{n}: \mathbf{L B A} \rightarrow \mathbf{G E R}_{n}^{\text {proj }} .
$$

One sees that $\mathfrak{L}_{n}(<m>)=0$ for all $m>n$ so that we get an SM-functor

$$
\mathfrak{L}_{n}: \mathbf{L B A}_{n} \rightarrow \mathbf{G E R}_{n}^{\text {proj }}
$$

This functor canonically extends to a SM-functor

$$
\mathfrak{L}_{n}: \mathbf{L B A}_{n}^{\text {proj }} \rightarrow \mathbf{G E R}_{n}^{\text {proj }} .
$$


The functors $\mathfrak{L}_{n}$ produce a map of projective systems

$$
\underline{\mathfrak{L}}: \underline{\mathrm{LBA}}^{\text {proj }} \rightarrow \underline{\mathrm{GER}}^{\text {proj }} .
$$

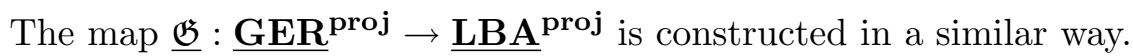

The natural transformations from 5.3.3,5.3.4 produce the natural transformations

$$
\mathrm{Id} \rightarrow \underline{\mathfrak{L} \mathfrak{G}} ; \mathrm{Id} \rightarrow \underline{\mathfrak{G} \mathfrak{L}}
$$

One can easily check that these transformations are weak equivalences. Therefore, the maps of projective systems of SMC $\underline{\mathfrak{L}}, \underline{\mathfrak{G}}$ are weak equivalences (because their compositions in both ways are weakly equivalent to the identity).

5.4.9. Conclusion. We have constructed a number of SM weak equivalences

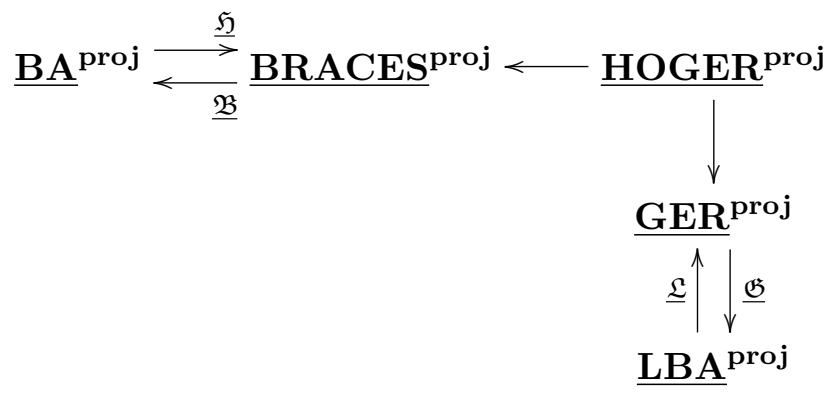

We have also constructed weak equivalences

$$
\begin{gathered}
\mathrm{Id} \rightarrow \underline{\mathfrak{B} \mathfrak{H}} ; \operatorname{Id} \rightarrow \underline{\mathfrak{G} \mathfrak{L} ; \operatorname{Id} \rightarrow \underline{\mathfrak{L} \mathfrak{G}} .} \\
\text { 6. } t \text {-STRUCTURES }
\end{gathered}
$$

We are going to endow each SMC in (15) with a $t$-strucrture [1]. Next, we will show that all the arrows in (15) are exact with respect to these $t$-structures.

6.0.10. Let us recall the definition of a $t$-structure on a dg-category $\mathcal{C}$. We assume that for every object $X \in \mathcal{C}$ and any integer $n$ there exists an object $X[n]$ which represents the functor

$$
U \mapsto \operatorname{hom}(U ; X)[n] .
$$

It follows that we have a natural isomorphism

$$
\operatorname{hom}(X[n] ; Y[m]) \stackrel{\sim}{\rightarrow} \operatorname{hom}(X ; Y)[m-n] .
$$

We define a t-structure on $\mathcal{C}$ as a collection of full subcategories $\mathcal{D}^{\leq n}, \mathcal{D}^{\geq n} \subset \mathcal{C}$ for all integers $n, \mathcal{D}^{\leq n}=\mathcal{D}^{\leq 0}[-n] ; \mathcal{D}^{\geq n}=D^{\geq n}[-n]$. The following properties should be satisfied:

$1 \mathcal{D}^{\leq 0} \subset \mathcal{D}^{\leq 1}$

$2 \mathcal{D}^{\geq 1} \subset \mathcal{D}^{\geq 0}$

3 For any $X \in \mathcal{D}^{\leq-1}$ and $Y \in \mathcal{D}^{\geq 0}, H^{0} \operatorname{hom}(X, Y)=0$;

4 For any $X$ in $C$ there exist objects $A \in \mathcal{D}^{\leq-1}, B \in \mathcal{D}^{\geq 0}$ and arrows $A \rightarrow X \rightarrow B$ whose composition is zero and such that for every $U \in \mathcal{C}$ the complex

$$
0 \rightarrow \operatorname{hom}(U, A) \rightarrow \operatorname{hom}(U, X) \rightarrow \operatorname{hom}(U, B) \rightarrow 0
$$

is acyclic. 


\subsubsection{The categories $\mathbf{B} \mathbf{A}_{n}^{\text {proj }}$.}

PROPOSITION 6.1. Every object in $\mathbf{B A}_{n}^{\wedge}$ admits a finite projective resolution centered in degrees $\leq 0$.

Proof. Let $F \in \mathbf{B A}_{n}^{\wedge}$. We have a quasi-isomorphism

$$
F \rightarrow \mathfrak{B H}(F)
$$

On the other hand we have a finite projective resolution $K \rightarrow \mathfrak{H}(F)$ (it is easy to see that every finite complex in $\mathbf{B R A C E S}{ }_{n}$ admits a finite projective resolution.) Thus we have a diagram

$$
F \rightarrow \mathfrak{B H}(F) \leftarrow \mathfrak{B}(K) .
$$

The complex $\mathfrak{B}(K)$ is finite and free and its only non-vanishing cohomology is the zeroth, this zeroth cohomology is isomorphic to $F$. It then follows that the subcomplex

$$
R F:=\tau_{\leq 0} \mathfrak{B}(K) \subset \mathfrak{B}(K)
$$

is finite and projective. Indeed, Let $N$ be the maximal number for which $K^{N} \neq 0$. If $N=0$ there is nothing to prove. If $N>0$, then, since $H^{N}(K)=0$, the map

$$
d_{N}: K^{N-1} \rightarrow K^{N}
$$

is surjective, hence splits, as all terms are projective. Let $K_{N-1}^{\prime}:=\operatorname{Ker} d_{N}$. We then know that $K_{N-1}^{\prime}$ is projective. If $N-1=0$ we are done; if not, we repeat the same procedure.

6.0.12. Set $\mathcal{D}^{\geq 0} \mathbf{B} \mathbf{A}_{n}^{\text {proj }}$ to consist of all objects whose cohomology is only in degrees $\geq 0$ and $\mathcal{D} \leq 0 \mathbf{B} \mathbf{A}_{n}^{\text {proj }}$ to consist of all objects whose cohomology is only in degrees $\leq 0$.

The axioms can be verified as follows.

1,2 - clear;

3 follows from the statement that any object from $\mathcal{D}^{\leq 0}$ admits a finite projective resolution whose all terms are in degree $\leq 0$;

4 Given an object $F$ let $G \rightarrow \tau_{\leq 0} F$ be a resolution. Then the cone of the map $G \rightarrow F$ is in $D^{>0}$. This is the required decomposition.

6.0.13. The core of this $t$-structure is $\mathbf{B} \mathbf{A}_{n}^{\wedge}$.

6.0.14. Tensor products on $\mathbf{B} \mathbf{A}_{n}$ and the projection maps $\mathbf{B} \mathbf{A}_{n} \rightarrow \mathbf{B} \mathbf{A}_{n-1}$ are right exact (i.e. preserve $\mathcal{D}^{\leq 0}$ ).

6.1. $t$-structre on $\mathbf{L B A} \mathbf{A}_{n}$. is introduced in the same way. Same results about the right exactness of the tensor product and projections do hold.

6.2. $t$-structure on $\mathbf{G E R}_{n}, \mathbf{B R A C E S}_{n}$. Let $C$ be a dg-category with $n+1$ objects $<0>,<1>$ $,<2>, \ldots,<n>$. Assume that:

$-\operatorname{hom}(<j>,<i>)=0$ if $j>i$;

$-\operatorname{hom}(<i>,<i>)$ is a finite dimensional semi-simple algebra.

- if $j \leq i$, then $\operatorname{hom}(\langle j\rangle,<i>)$ is a finite complex whose all non-zero terms are in degrees from 0 to $j-i$ inclusive;

The categories $\mathbf{G E R}_{n}^{\text {op }}, \mathbf{B R A C E S}_{n}^{\text {op }}$ are such.

Let us construct a $t$-structure on the category $E$ of finite projective complexes of functors $C \rightarrow$ vect. 
We set $\mathcal{D}^{\geq 0} E$ to consist of all objects $X$ such that $H^{j} X(<i>) \neq 0$ only if $j \geq-i$. We set $\mathcal{D} \leq 0$ to consist of all complexes of projective modules $P$ generated by several elements from several complexes $P(<i>)$ in degree $\leq-i$.

Let us prove it is indeed a $t$-structure.

$1,2,3$ are obvious. Let us pass to 4 . We will use the cone construction; in order to avoid an umbiguity, let us fix the agreements: given a map of objects $f: X \rightarrow Y, X, Y \in E$ We define an object Cone $(f)$ by setting

$$
\text { Cone }(f)(<i>)^{n}:=Y(<i>)^{n} \oplus X(<i>)^{n+1} .
$$

Let $y \in Y(<i>)^{n} ; x \in X(<i>)^{n+1}$ we then set

$$
d(y \oplus x)=y^{\prime} \oplus x^{\prime}
$$

where $y^{\prime}=d y+(-1)^{n} f(x) ; x^{\prime}=d x$.

In particular we have natural maps

$$
Y \rightarrow \text { Cone }(f) \rightarrow X[1]
$$

whose composition is zero.

Let us now proceed to the proof.

Let $E_{i} \subset E$ be the full subcategory consisting of all objects $X$ with $X(<j>)=0$ whenever $j+i<n$, so that $E_{0} \subset E_{1} \subset E_{2} \subset \cdots E_{n}=E$.

Let us prove our statement by induction.

1) if $X \in E_{0}$, the statement is clear: indeed, the category $E_{0}$ is that of $\left.\operatorname{End}(<n\rangle\right)$-modules. $\mathcal{D}^{\geq 0} E \cap E_{0}$ consists of all modules in degrees $\geq-n$, and the category $D^{\leq 0} E \cap E_{0}$ consists of all modules in degrees $\leq-n$, so $\tau_{\leq-n-1} X \in \mathcal{D}^{<0} ;$ and $\tau_{\geq-n} X \in \mathcal{D}^{\geq 0}$.

2) Induction step. Let $X \in E_{i}$ so that $X(<j>) \neq 0$ only if $j \geq n-i$.

Consider the complex $X(<n-i>)$. This complex has a natural structure of a $\operatorname{dg} \operatorname{End}(<n-i>)$ module. Let us decompose

$$
\tau_{\leq i-n-1} X(<n-i>) \rightarrow X(<n-i>) \rightarrow \tau_{\geq i-n} X(<n-i>),
$$

where the truncations are taken in the category of complexes of $\operatorname{End}(<n-i>)$-modules.

Let $L \in E_{i}$ be the object freely generated by the $\operatorname{End}(<n-i>)$-module $\left.\tau_{\leq i-n-1} X(<n-i\rangle\right)$. Let $W: C \rightarrow$ complexes be defined as follows:

$-W(<k>)=0$ for all $k \neq n-i$;

$-W(<n-i>)=\tau_{\geq i-n} X(<n-i>)$.

The $C$-action is as follows: all complexes $\operatorname{hom}_{C}\left(<k_{1}>;<k_{2}>\right)$ act by zero except $\operatorname{hom}_{C}(<$ $n-i>;<n-i>)=\operatorname{End}(<n-i>)$ which has a natural action on $W$.

We then have naturally defined maps

$$
L \stackrel{i_{L}}{\rightarrow} X \stackrel{p_{W}}{\rightarrow} W
$$

The following properties are the case:

$-p_{W} i_{L}=0$

$-L \in \mathcal{D}^{<0} E$

- Let $W^{\prime}$ be any object from $E_{i}$ such that there exists a quasi-isomorphism $W^{\prime} \rightarrow W$ in the category of dg functors $C \rightarrow$ complexes, then $W^{\prime} \in \mathcal{D}^{\geq 0}$. 
- The following sequence of complexes

$$
0 \rightarrow L(<n-i>) \rightarrow X(<n-i>) \rightarrow W(<n-i>) \rightarrow 0
$$

is short exact.

Let us define the functor $A: C \rightarrow$ complexes by setting $A(<k>)$ to be the total complex of the bi-complex

$$
0 \rightarrow L(<k>) \rightarrow X(<k>) \rightarrow W(<k>) \rightarrow 0,
$$

where $L$ is in the horizontal cohomological degree 0 .

We have natural maps $\pi_{L}: A \rightarrow L$ and $\iota_{W}: W[-2] \rightarrow A$ satisfying $\pi_{L} \iota_{W}=0$. The object $X$ is canonically quasi-isomorphic to

$$
X^{\prime}:=\text { Cone }\left[W[-2] \stackrel{\iota_{W}}{\rightarrow} \operatorname{Cone}\left(A \stackrel{\pi_{L}}{\rightarrow} L\right)\right]
$$

Since $A(\langle n-i\rangle)$ is acyclic, $A$ is quasi-isomorphic to an object from $E_{i-1}$, hence, by the induction assumption, the statement of the Lemma is applicable to $A[1]$. Thus there is a $U \in \mathcal{D}^{<0}$ and a map $U \stackrel{\iota_{U}}{\rightarrow} A[1]$ such that the cone of $\iota_{U}: U \rightarrow A[1]$ is quasi-isomorphic to an object from $\mathcal{D}^{\geq 0}$. Consider the through map

$$
U[-1] \stackrel{\iota_{U}}{\longrightarrow} A \stackrel{\pi_{L}}{\longrightarrow} L
$$

Let $V$ be the cone of this through map. Since $U[-1] \in \mathcal{D}^{\leq 0}$ and $L \in \mathcal{D}^{<0}$, we have $V \in \mathcal{D}^{<0}$.

We are going to define an arrow $\phi: V \rightarrow X^{\prime}$ such that Cone $\phi \in \mathcal{D}^{\geq 0}$. By doing so we will prove the statement.

The arrow $\phi$ will be constructed as a composition

$$
\phi: V \rightarrow \operatorname{Cone}(A \rightarrow L) \rightarrow X^{\prime}
$$

The left arrow is defined by the diagram

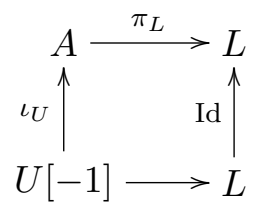

where $\iota_{U}, \pi_{L}$ are as in (17). The arrow $\operatorname{Cone}(A \rightarrow L) \rightarrow X^{\prime}$ is defined as the identity embedding onto Cone $(A \rightarrow L)$.

It is not hard to see that Cone $\phi$ is canonically quasi-isomorphic to the cone of the following composition:

$$
W[-1] \stackrel{\iota_{W}}{\rightarrow} A[1] \rightarrow \operatorname{Cone}(U \rightarrow A[1])
$$

Next, we know that $\operatorname{Cone}(U \rightarrow A[1])$ is quasi-isomorphic to an object from $\mathcal{D}^{\geq 0}$ and so is $W$, this implies that the cone is also quasi-isomorphic to an object from $\mathcal{D}^{\geq 0}$. Therefore, Cone $(\phi) \in \mathcal{D}^{\geq 0}$ as we wanted.

PROPOSITION 6.2. The natural projections $\mathbf{G E R}_{n} \rightarrow \mathbf{G E R}_{m} ; \mathbf{H O G E R}_{n} \rightarrow \mathbf{H O G E R}_{m}$; BRACES $_{n} \rightarrow$ BRACES $_{m}$ are right exact. The tensor products on these categories are right exact.

Proof. Straightforward 
6.2.1.

PROPOSITION 6.3. The weak equivalences $\mathfrak{H}_{n} ; \mathfrak{B}_{n}, \mathfrak{L}_{n}, \mathfrak{G}_{n}$ are exact, i.e. preserve $\mathcal{D}^{\geq 0}, \mathcal{D}^{\leq 0}$.

Proof. Since all the functors listed are weak equivalences, it suffices to check they preserve $\mathcal{D} \leq 0$. As all the functors commute with shifts, $\mathcal{D}^{\leq i}$ will be preserved as well.

The categories $\mathcal{D}^{\geq i}$ are uniquely determined by the condition :

$X \in \mathcal{D}^{\geq i}$ iff $H^{<0} \operatorname{hom}(Y, X)=0$ for all $Y \in \mathcal{D}^{\leq i}$.

Hence, $\mathcal{D}^{\geq i}$ must be preserved by any weak equivalence as long as $\mathcal{D}^{\leq 0}$ is preserved

The preservation of $\mathcal{D}^{\leq 0}$ follows from the definitions. Indeed, we have:

$X \in \mathcal{D}^{\leq 0} \mathbf{B} \mathbf{A}_{n}^{\text {proj }}$ iff $X$ is a retraction of a finite complex of finitely generated free objects whose all generators have grading $\leq 0$. Same definition works for $\mathcal{D}^{\leq 0} \mathbf{L B} \mathbf{A}_{n}^{\text {proj}}$;

$X \in \mathcal{D}^{\leq 0} \mathbf{B R A C E S}{ }_{n}^{\text {proj }}$ iff $X$ is a retraction of a finite complex of finitely generated free objects whose all generators from $X(<k>)$ have grading $\leq-k$; same definition for $\mathcal{D}^{\leq 0}$ ger $_{n}^{\text {proj }}$.

We can now check immediately that

$$
\mathfrak{H}_{n}\left(h_{<1>}\right) \in \mathcal{D}^{\leq 0} \mathbf{B R A C E S} \mathbf{S}_{n}^{\text {free }},
$$

this follows from $(9)$

Therefore, $\mathfrak{H}_{n}\left(h_{<k>}\right)=\mathfrak{H}_{n}\left(h_{<1>}\right)^{\otimes k} \in \mathcal{D}^{\leq 0} \mathbf{B R A C E S}_{n}^{\text {free }}$ because the tensor product is right exact. This fact easily implies that $\underline{\mathfrak{H}}_{n}\left(\mathcal{D}^{\leq 0}\right) \subset \mathcal{D}^{\leq 0}$.

Let us show that $\mathfrak{B}_{n} \mathcal{D}^{\leq 0} \subset \mathcal{D}^{\leq 0}$. Since the weak equivalence $\underline{\mathfrak{H}}_{n}$ is exact, it follows that the functor

$$
\mathfrak{H}_{n}: \mathcal{D}^{\leq 0} \mathbf{B A}_{n}^{\text {proj }} \rightarrow \mathcal{D}^{\leq 0} \mathbf{B R A C E S}_{n}^{\text {proj }}
$$

is essentially surjective. Therefore, it suffices to prove that

$$
\mathfrak{B}_{n} \mathfrak{H}_{n} \mathcal{D}^{\leq 0} \mathbf{B A}_{n}^{\text {proj }} \subset \mathcal{D}^{\leq 0} \mathbf{B A}_{n}^{\text {proj }}
$$

This fact follows immediately from the weak equivalence $\mathrm{Id} \rightarrow \mathfrak{B}_{n} \mathfrak{H}_{n}$ (see 5.1).

The proof for the functors $\underline{\mathfrak{L}}, \underline{\mathfrak{G}}$ goes along the same lines.

\section{PROOF OF THEOREM 3.3}

The above proven propositions show that the equivalences $\mathfrak{H}_{n} ; \mathfrak{B}_{n}, \mathfrak{L}_{n}, \mathfrak{G}_{n}$ induce equivalences of the cores of the corresponding projective systems of SMC. Given a dg-category $C$ with a $t$ structure, let $[C]$ be its core. Given an exact dg SM-functor $F: C \rightarrow D$ between $S M$-categories with $t$-structures, let $[F]:[C] \rightarrow[D]$ be the induced equivalence of the cores.

Thus we have a chain of SM-equivalences

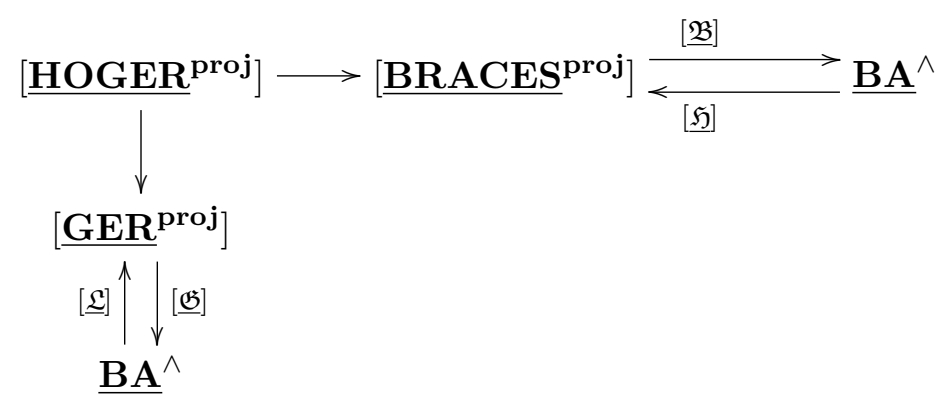


The arrows $[\underline{\mathfrak{B}}]$ and $[\mathfrak{H}]$ are mutually quasi-inverse (i.e. their compositions are isomorphic to the identity), as well as $[\underline{\mathfrak{G}}]$ and $[\mathfrak{L}]$.

This gives rise to a unique (up-to a unique isomorphism) equivalence

$$
\mathrm{Q}: \underline{\mathbf{B A}}^{\wedge} \rightarrow \underline{\mathbf{L B A}}^{\wedge}
$$

as stated in the theorem

\section{DePEndence On THE CHOICE OF A QUASI-ISOMORPHISM hoger $\rightarrow$ braces}

Our construction of the equivalence of systems $\underline{\mathbf{B A}}^{\wedge} \rightarrow \underline{\mathbf{L B A}}^{\wedge}$ depends on the choice of a quasi-isomorphism of operads $U:$ hoger $\rightarrow$ braces. Denote the corresponding equivalence

$$
\mathbf{Q}_{U}: \underline{\mathbf{B A}}^{\wedge} \rightarrow \underline{\mathbf{L B A}}^{\wedge} .
$$

We will answer the following question: given two different quasi-isomorphisms $U_{1}, U_{2}:$ hoger $\rightarrow$ braces, are the equivalences $\mathbf{Q}_{U_{1}}$ and $\mathbf{Q}_{U_{2}}$ isomorphic?

The answer to this question can be conveniently given in terms of the derived category of dgoperads coming from the closed model structure on it [10]. Denote this derived category by $\mathrm{D}(\mathrm{op})$

We then have the set of isomorphisms $\operatorname{Iso}_{\mathrm{D}(\mathrm{op})}$ (ger, braces). The quasi-isomorphisms $U_{1}, U_{2}$ define elements $\bar{U}_{1}, \bar{U}_{2} \in \mathrm{Iso}_{\mathrm{D}(\mathrm{op})}$ (ger, braces).

In order to formulate a precise result, we need a one more thing. Given $x \in \mathbf{k}^{\times}$, let $\iota_{x}:$ hoger $\rightarrow$ hoger be the automorphism which acts on $\operatorname{hoger}(n)$ as the dilation by $x^{1-n}$. This way, we have a map

$$
\mathbf{k}^{\times} \rightarrow \mathrm{IsO}_{\mathrm{D}(\mathrm{op})}(\text { ger, ger }) .
$$

The latter group acts on $\operatorname{Iso}_{\mathrm{D}(\mathrm{op})}$ (ger, braces), hence the induced $\mathbf{k}^{\times}$-action on $\operatorname{Iso}_{\mathrm{D}(\mathrm{op})}$ (ger, braces). Let $\sim$ be the equivalence relation on $\mathrm{Iso}_{\mathrm{D}(\mathrm{op})}\left(\right.$ ger, braces) generated by this $\mathbf{k}^{\times}$-action.

We will prove:

THEOREM 8.1. 1) If $\bar{U}_{1} \sim \bar{U}_{2}$, then $\mathbf{Q}_{U_{1}}$ is isomorphic to $\mathbf{Q}_{U_{2}}$;

2) If $\bar{U}_{1} \nsim \bar{U}_{2}$ then $\mathbf{Q}_{U_{1}}$ and $\mathbf{Q}_{U_{2}}$ are not isomorphic. Furthermore, there exists an $N$ such that the induced symmetric monoidal functors

$$
\mathbf{Q}_{U_{1}, N}, \mathbf{Q}_{U_{2}, N}: \mathbf{B} \mathbf{A}_{N}^{\wedge} \rightarrow \mathbf{L B} \mathbf{A}_{N}^{\wedge}
$$

are not isomorphic.

Remark 1 If $\mathbf{Q}_{U_{1}}$ and $\mathbf{Q}_{U_{2}}$ are isomorphic, then the set of isomorphisms $\mathbf{Q}_{U_{1}} \rightarrow \mathbf{Q}_{U_{2}}$ is pretty large and seems to be parameterized by the set of all homotpy classes of homotopies between the quasi-isomorphisms $U_{1}, U_{2}:$ hoger $\rightarrow$ braces which is a torsor over the pro-nilpotent group $\operatorname{Exp}\left(H^{-1} F^{1} \mathfrak{g}\right)$, where $\mathfrak{g}$ is as in Appendix 2 .

Remark 2 Using the statement 2) of the theorem we can show that given $U_{1}, U_{2}$ producing nonequivalent elements in $\mathrm{Iso}_{\mathrm{D}(\mathrm{op})}$ ger, one can construct a conilpotent Lie bialgebra in some SMC whose quantizations using $\mathbf{Q}_{U_{1}}$ and $\mathbf{Q}_{U_{2}}$ are non-isomorphic.

As an appropriate SMC, we take the category of all functors from $\mathbf{L B A} \mathbf{A}_{N}^{\text {op }}$ to the category of vector spaces (it is larger than $\mathbf{L B} \mathbf{A}_{N}^{\wedge}$; we have to take such a larger category in order to satisfy the conditions from 4.1.2). Denote this category by $\mathcal{C}_{N}$.

We see that the representing object $h_{<1>} \in \mathcal{C}_{N}$, of $<1>\in \mathbf{L B} \mathbf{A}_{N}$, is a conilpotent Lie bialgebra because $h_{<1>}^{\otimes M}=0$ for all $M>N$. Denote this conilpotent Lie bialgebra by $\mathfrak{a}$. 
The quantization functors produce a pair of conilpotent BA-algebras in $\mathcal{C}_{n}: \mathfrak{m}_{i}:=\mathbf{Q}_{U_{i}} \mathfrak{a}, i=1,2$ Let us show this BA-algebras are not isomorphic. Indeed, the opposite assumption implies that we have SM-monoidal isomorphism of the functors $\mathbf{Q}_{U_{i}, N}, i=1,2$, which, for $N$ large enough, contradicts to the statement 2) of the theorem.

8.1. proof of the first part of the Theorem. Suppose that $U_{1}, U_{2}:$ hoger $\rightarrow$ braces produce equivalent elements in $\mathrm{Iso}_{\mathrm{D}(\mathrm{op})}$ (ger, braces). By definition, there exists an $x \in \mathbf{k}^{\times}$such that $U_{1}$ and $U_{2}^{\prime}:=U_{2} \iota_{x}$ produce the same element in $\operatorname{Iso}_{\mathrm{D}(\mathrm{op})}$ (ger, braces).

Step 1 Let us first prove that $\mathbf{Q}_{U_{2}}$ is isomorphic to $\mathbf{Q}_{U_{2} \iota_{x}}$. Indeed, the induced SM functors

$$
U_{2}, U_{2}^{\prime}: \text { HOGER } \rightarrow \text { BRACES }
$$

are isomorphic: the isomorphism $i: U_{2} \rightarrow U_{2}^{\prime}$ is defined by setting

$$
i(<l>): U_{2}(<l>) \rightarrow U_{2}^{\prime}(<l>)
$$

to be the dilation $x^{l} \operatorname{Id}$ on $<l>=U_{2}(<l>)=U_{2}^{\prime}(<l>)$. This isomorphism induces a quasiisomorphism $\mathbf{Q}_{U_{2}} \rightarrow \mathbf{Q}_{U_{2}^{\prime}}$ in the obvious way.

Step 2 Let us now prove that the functors $\mathbf{Q}_{U_{1}}$ and $\mathbf{Q}_{U_{2}^{\prime}}$ are isomorphic. We will write $U_{2}$ instead of $U_{2}^{\prime}$. Our task then reduces to showing that $\mathbf{Q}_{U_{1}}$ and $\mathbf{Q}_{U_{2}}$ are isomorphic as long as $U_{1}$ and $U_{2}$ produce the same element in $\mathrm{Iso}_{\mathrm{D}(\mathrm{op})}$ (ger, braces).

According to Quillen, this means that there exists a map

$$
P: \text { hoger } \rightarrow \text { braces }[t, d t]
$$

such that $r_{1} P=U_{1} ; r_{2} P=U_{2}$.

Since the operad hoger is concentrated in degrees $\leq 0$, therefore, the map $P$ takes values in the suboperad $\tau_{\leq 0}$ braces $[t, d t]$. Denote the latter suboperad by o so that we have a diagram

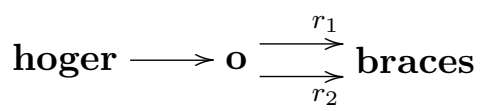

Let $\mathbf{O}$ be the PROP generated by $\mathbf{o}$, let $\mathbf{O}_{n}$ be the quotient of $\mathbf{O}$ by the ideal generated by $\operatorname{Id}_{N}$, $N>n$. Let $\underline{\mathbf{O}}$ be the corresponding projective system of PROPs.

Let $i:$ braces $\rightarrow$ braces $[t, d t]$ be the constant embedding so that $r_{1} i=r_{2} i$. We denote by the same letter the induced embedding $i:$ braces $\rightarrow \mathbf{o}$.

We then have an induced diagram of projective systems of PROPs:

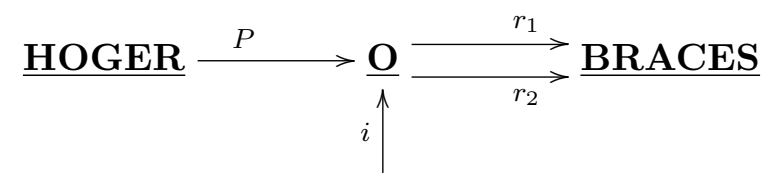

\section{BRACES}

Let us define a $t$-structure on $\mathbf{O}_{N}$ according to the recipe of Sec. 6.2 (note that the category $\mathbf{O}_{N}$ satisfies all the conditions therein). We then see that all the arrows are $t$-exact, hence induce an equivalence of cores. We have a natural isomorphism $\left[r_{1} i\right]=\left[r_{2} i\right]$. Since $i$ is an equivalence, there is a quasi-inverse equivalence $j:[\underline{\mathbf{O}}] \rightarrow[$ BRACES $]$, and we have equivalences $\left[r_{1} i j P\right]=\left[r_{2} i j P\right]$; $\left[r_{1} i j P\right]=\left[r_{1} P\right]=\left[U_{1}\right] ;\left[r_{2} i j P\right]=\left[r_{2} P\right]=\left[U_{2}\right]$, so we have established an equivalence $\left[U_{1}\right] \rightarrow\left[U_{2}\right]$. This readily implies that $\mathbf{Q}_{U_{1}}$ and $\mathbf{Q}_{U_{2}}$ are isomorphic. 
8.2. proof of the second part of the theorem. According to the Appendix 2 (Proposition 10.6, there exists an $N$ such that the images of $U_{1}, U_{2}$ in

Iso $_{D(n \text {-op })}\left(\right.$ ger $_{\leq N} ;$ braces $\left._{\leq N}\right)$ are not conjugated by any $\iota_{x}: \operatorname{hoger}_{\leq N} \rightarrow$ hoger $_{\leq N}, x \neq 0$. Let us prove that the SM-functors $\mathbf{Q}_{U_{1}, N}$ and $\mathbf{Q}_{U_{2}, N}$ are non-isomorphic. We need an auxiliary construction.

8.2.1. Let $C$ be a dg SMC and $X, Y$ be a pair of homotopy equivalent objects. This implies that there exist elements $\bar{f} \in H^{0} \operatorname{hom}_{C}(X, Y) ; \bar{g} \in H^{0} \operatorname{hom}_{C}(Y, X)$ such that $\bar{f} \bar{g}=\overline{\operatorname{Id}_{Y}} ; \bar{g} \bar{f}=\overline{\operatorname{Id}_{X}}$, in particular, $\bar{g}$ is uniquely determined by $\bar{f}$. Given such an $\bar{f}$, we will construct an isomorphism in $D(n-\mathrm{op})$

$$
I(\bar{f}): \operatorname{full}(X)_{\leq n} \rightarrow \operatorname{full}(Y)_{\leq n}
$$

Note that $I(\bar{f})$ will only depend on $\bar{f}$.

First of all let $C^{\prime}$ be the dg-category of finite complexes in $C$ and let us identify $C$ with the corresponding full subcategory in $C^{\prime}$. The latter category is closed under taking cones.

Pick a representative $f: X \rightarrow Y$ whose class in $H^{0} \operatorname{hom}(X, Y)$ is $\bar{f}$, and consider the following object in $C^{\prime}$ :

$$
K_{f}:=K:=\operatorname{Cone}\left(X \oplus Y \stackrel{f \oplus \operatorname{Id}_{X}}{\rightarrow} Y\right)[-1]
$$

We have natural projections $p_{X}: K \rightarrow X ; p_{Y}: K \rightarrow Y$. As $f$ is a quasi-isomorphism, it easily follows that both $p$ and $q$ are split quasi-isomorphisms. (i.e. there exist $i_{X}: X \rightarrow K ; i_{Y}: Y \rightarrow K$ such that $p_{X} i_{X}=\operatorname{Id}_{X} ; p_{Y} i_{Y}=\operatorname{Id}_{Y}$. This, in turn, implies that there exist isomorphisms

$$
X \oplus R_{X} \rightarrow K ; \quad Y \oplus R_{Y} \rightarrow K
$$

such that $R_{X}, R_{Y}$ are quasi-isomorhic to 0 and the maps $p_{X}, p_{Y}, i_{X}, i_{Y}$ correspond under these isomorphisms to the natural embeddings and projections of $X, Y$ as direct summands.

We will now define a pair of sub-operads of full $(K)$ as follows.

Let $\mathbf{o}_{X}(n)$ be the kernel of the following arrow

$$
A_{X}: \operatorname{hom}\left(K^{\otimes n} ; K\right) \oplus \operatorname{hom}\left(X^{\otimes n} ; X\right) \stackrel{A_{X}^{1}-A_{X}^{2}}{\rightarrow} \operatorname{hom}\left(K^{\otimes n} ; X\right)
$$

whose components are defined as follows:

$$
A_{X}^{1}: \operatorname{hom}\left(K^{\otimes n} ; K\right) \rightarrow \operatorname{hom}\left(K^{\otimes n} ; X\right)
$$

by post-composition with $p: K \rightarrow X$;

$$
A_{X}^{2}: \operatorname{hom}\left(X^{\otimes n} ; X\right) \rightarrow \operatorname{hom}\left(K^{\otimes n} ; X\right)
$$

by pre-composition with $p$.

We then have natural maps of operads

$$
\operatorname{full}(X) \leftarrow \mathbf{o}_{X} \rightarrow \operatorname{full}(K)
$$

which are quasi-isomorphisms as easily follows from the isomorphism $K=X \oplus R_{X}$ and exactness of the tensor product.

In the same way, we construct a diagram

$$
\operatorname{full}(Y) \leftarrow \mathbf{o}_{Y} \rightarrow \operatorname{full}(K),
$$

where $\mathbf{o}_{Y}(n)$ is defined as the kernel of 


$$
A_{Y}: \operatorname{hom}\left(K^{\otimes n} ; K\right) \oplus \operatorname{hom}\left(Y^{\otimes n} ; Y\right) \stackrel{A_{Y}^{1}-A_{Y}^{2}}{\rightarrow} \operatorname{hom}\left(K^{\otimes n} ; Y\right)
$$

where

$$
\begin{aligned}
& A_{Y}^{1}: \operatorname{hom}\left(K^{\otimes n} ; K\right) \rightarrow \operatorname{hom}\left(K^{\otimes n} ; Y\right) ; \\
& A_{X}^{2}: \operatorname{hom}\left(Y^{\otimes n} ; Y\right) \rightarrow \operatorname{hom}\left(K^{\otimes n} ; Y\right)
\end{aligned}
$$

are given by post-composing and pre-composing with $p_{Y}$.

We then have a diagram of quasi-isomorphisms

$$
\operatorname{full}(X) \rightarrow \operatorname{full}(K) \rightarrow \operatorname{full}(Y)
$$

which defines an isomorphism

$$
I_{f}: \operatorname{full}(X)_{\leq n} \rightarrow \operatorname{full}(Y)_{\leq_{n}}
$$

in $D\left(n\right.$-op). Let $f^{\prime}: X \rightarrow Y$ be another representative of $\bar{f}$. Therefore, we have $f^{\prime}-f=d \gamma$, where

$$
\gamma \in \operatorname{hom}^{-1}(X, Y) \text {. }
$$

We can construct an isomorphism $K_{f} \rightarrow K_{f^{\prime}}$ defined as follows:

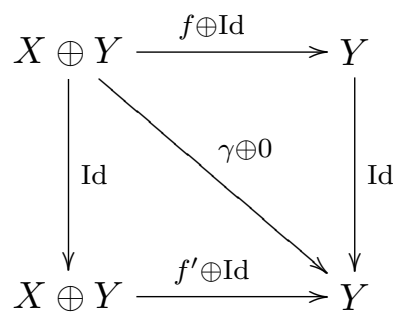

This isomorphism commutes with the projections $K_{f} \rightarrow X, Y ; K_{f^{\prime}} \rightarrow X, Y$, therefore, it induces a commutative diagram

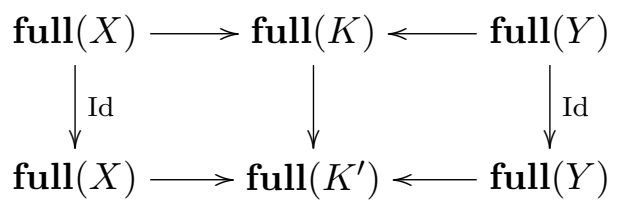

in which all arrows are quasi-isomorphisms.

This diagram implies that $I_{f}=I_{f^{\prime}}$.

8.2.2. Let $\mathcal{P}, \mathcal{C}$ be $\operatorname{dg}$ SMC. Suppose that we are given two symmetric monoidal maps

$$
\mathcal{P} \underset{i_{2}}{\stackrel{i_{1}}{\longrightarrow}} \mathcal{C}
$$

and a quasi-isomorphism $i_{1}(X) \rightarrow i_{2}(X)$ for some $X \in \mathcal{P}$. We then get a couple of arrows in $D(n$-op)

$$
\operatorname{full}_{\mathcal{P}}(X)_{\leq n} \rightrightarrows \operatorname{full}_{\mathcal{C}}\left(i_{2}(X)\right)_{\leq n} .
$$

Indeed, we have maps of operads

$$
\text { full }_{\mathcal{P}}(X)_{\leq n} \rightarrow \operatorname{full}_{\mathcal{C}}\left(i_{1}(X)\right)_{\leq n} ; \operatorname{full}_{\mathcal{P}}(X)_{\leq n} \rightarrow \operatorname{full}_{\mathcal{C}}\left(i_{2}(X)\right)_{\leq n}
$$


and an isomorphism in $D\left(n\right.$-op) full fu $_{\mathcal{C}}\left(i_{1}(X)\right)_{\leq n} \rightarrow$ full $_{\mathcal{C}}\left(i_{2}(X)\right)_{\leq n}$, whence a pair of arrows as promised.

8.2.3. Suppose we have a diagram

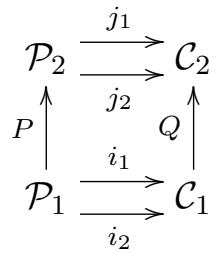

of SMC and SM functors; let us also assume that we are given isomorphisms of SM-functors:

$$
\begin{aligned}
& j_{1} P \stackrel{\sim}{\rightarrow} Q i_{1} ; \\
& j_{2} P \stackrel{\sim}{\rightarrow} Q i_{2} .
\end{aligned}
$$

Let $X_{1} \in \mathcal{P}_{1}$ and $X_{2}:=P\left(X_{1}\right)$. Suppose we have a homotopy equivalence $\xi: i_{1}\left(X_{1}\right) \rightarrow i_{2}\left(X_{1}\right)$. We then have an induced homotopy equivalence $Q(\xi): j_{1}\left(X_{2}\right) \rightarrow j_{2}\left(X_{2}\right)$.

It is clear that all these data produce a commutative diagram in $D(n$-op):

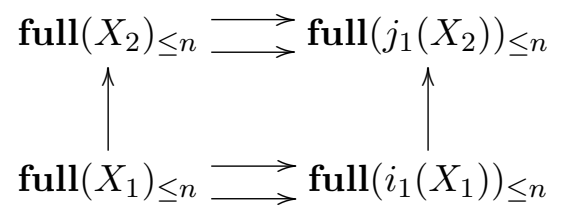

Assume $P, Q$ are homotopy equivalences, then the top horizontal arrows in (20) are equivalent iff so are the bottom horizontal arrows.

8.2.4. As explained above, we have SM maps

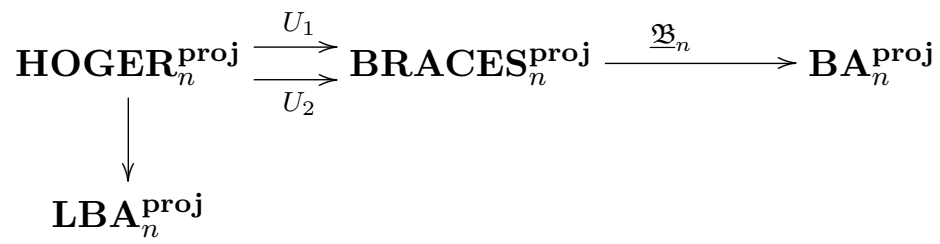

Denote the composition of the horizontal arrows by

$$
\mathbb{H}_{U_{1}}, \mathbb{H}_{U_{2}}: \text { HOGER }{ }_{n}^{\text {proj }} \rightarrow \text { BA }_{n}^{\text {proj }}
$$

denote the vertical arrow by

$$
\mathbb{L}: \text { HOGER }_{n}^{\text {proj }} \rightarrow \text { LBA }_{n}^{\text {proj }} .
$$

As was explained above, this diagram induces a pair of SM equivalences of the cores:

$$
\mathbf{Q}_{U_{1}}, \mathbf{Q}_{U_{2}}: \mathbf{B A}_{n}^{\wedge} \rightarrow \mathbf{L B A}_{n}^{\wedge}
$$

Let us make these equivalences more expicit. To this end let us choose $Z_{1}, Z_{2} \in$ HOGER ${ }_{n}^{\text {proj }}$ such that

$$
\mathbb{H}_{i}\left(Z_{i}\right) \cong h_{<1>}
$$

for $i=1,2$.

here $\cong$ means "quasi-isomorphic". Such objects $Z_{i}$ do exist because $\mathbb{H}_{i}$ are weak equivalences. 
Since $\mathbb{H}_{i}$ are exact functors, $Z_{i} \in \mathcal{D}^{\geq 0} \cap \mathcal{D}^{\leq 0}$, therefore, $Z_{i}$ define objects $\left[Z_{i}\right]$ in the core of

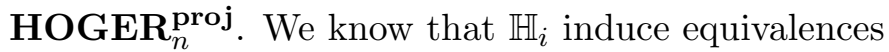

$$
\left[\mathbb{H}_{i}\right]:\left[\mathbf{H O G E R}_{n}^{\text {proj }}\right] \rightarrow\left[\mathbf{B A}_{n}^{\text {proj }}\right]
$$

and we have isomorphisms $\left[\mathbb{H}_{i}\right]\left[Z_{i}\right] \cong\left[h_{<1>}\right]$ induced by $(21)$. As $\left[h_{<1>}\right]$ ia a $\mathbf{B A}_{n}$-algebra, we have an induced $\mathbf{B A}_{n}$-structure on $\left[Z_{i}\right]$.

By definition, we have a canonical isomorphism in $\left[\mathbf{L B A}^{\mathbf{p r o j}}\right]$ :

$$
\left.\mathbf{Q}_{U_{i}, n}\left(h_{<1>}\right) \cong[\mathbb{L}]\left(\left[Z_{i}\right]\right)\right]
$$

The $\mathbf{B A}_{n}$-algebra structure on $h_{<1>}$ induces $\mathbf{B} \mathbf{A}_{n}$-structures on $\mathbf{Q}_{U_{i}, n}\left(h_{<1>}\right), i=1,2$. For each $i=1,2$, these structures, , upon the identification (22), coincide with the $\mathbf{B} \mathbf{A}_{n}$-structures induced by $[\mathbb{L}]$ from the $\mathbf{B} \mathbf{A}_{n}$-structures on $\left[Z_{i}\right]$.

On the other hand, by our assumption, the SM functors $\mathbf{Q}_{U_{i}, n}, i=1,2$ are isomorophic. This means that the $\mathbf{B A}_{n}$-algebras $\mathbf{Q}_{U_{i}, n}\left(h_{<1>}\right), i=1,2$ are also isomorophic, hence, the $\mathbf{B} \mathbf{A}_{n}$-algebras $[\mathbb{L}]\left[Z_{i}\right]$ are isomorphic as well. Since $[\mathbb{L}]$ is a $S M$ equivalence, the $\mathbf{B} \mathbf{A}_{n}$-algebras $\left[Z_{i}\right]$ are also isomorphic. Let $f:\left[Z_{1}\right] \rightarrow\left[Z_{2}\right]$ be the isomorphism.

Let us paraphrase this statement. Let

$$
\operatorname{FULL}\left(\left[Z_{1}\right],\left[Z_{2}\right]\right) \subset\left[\operatorname{HOGER}_{n}^{\text {proj}}\right]
$$

be the full SM subcategory consisting of all tensor powers $\left[Z_{1}\right]^{\otimes K_{1}} \otimes\left[Z_{2}\right]^{\otimes K_{2}}$ (for all $K_{1}, K_{2}$ ).

Let

$$
I_{k}: \mathbf{F U L L}\left(\left[Z_{k}\right]\right) \rightarrow \mathbf{F U L L}\left(\left[Z_{1}\right],\left[Z_{2}\right]\right)
$$

be the obvious embeddings.

Using the isomorphism $f$ we can construct the SM equivalence

$$
P: \mathbf{F U L L}\left(\left[Z_{1}\right],\left[Z_{2}\right]\right) \rightarrow \mathbf{F U L L}\left(\left[Z_{1}\right]\right)
$$

according to the following rules:

1) $P I_{1}=\operatorname{Id}_{\mathbf{F U L L}\left(\left[Z_{1}\right]\right)}$

2) $P\left(\left[Z_{2}\right]\right)=\left[Z_{1}\right]$

3) $P(f)=\operatorname{Id}_{\left[Z_{1}\right]}$

The functors $\mathbb{H}_{k}$ produce isomorphisms

$$
J_{k}: \mathbf{F U L L}\left(\left[Z_{k}\right]\right) \rightarrow \mathbf{F U L L}\left(h_{<1>}\right)=\mathbf{B A}_{n}
$$

The fact that $f$ is an isomorphism of $\mathbf{B} \mathbf{A}_{n}$-algebras simply means that

$$
J_{1} P I_{k} J_{k}^{-1}=\operatorname{Id}_{\mathbf{B} \mathbf{A}_{n}}
$$

for $k=1,2$.

Next, we observe that there exists an element $\bar{T} \in \operatorname{Aut}_{D(n \text {-op })}\left(\right.$ ger $\left._{\leq n}\right)$ such that $\overline{U_{1}}=\overline{U_{2} T}$. Let $T: \operatorname{hoger}_{\leq N} \rightarrow \operatorname{hoger}_{\leq N}$ be a representative of $\bar{T}$. It follows that $U_{1}$ and $U_{2} T$ produce the same element in $\operatorname{Iso}_{D(n \text {-op })}$ (ger, braces) hence, without loss of generality one can assume $U_{1}=U_{2} T$. It follows that that $\bar{T} \neq \overline{\iota_{x}}$ for all $x \in \mathbf{k}^{\times}$. Let $\mathcal{T}: \mathbf{H O G E R}_{n}^{\text {proj }} \rightarrow$ HOGER $_{n}^{\text {proj }}$ be the isomorphism induced by $T$.

It follows that one can choose $Z_{2}=\mathcal{T}\left(Z_{1}\right)$. Indeed, in this case

$$
\mathbb{H}_{U_{2}}\left(\mathcal{T}\left(Z_{1}\right)\right)=\underline{\mathfrak{B}}_{n} U_{2} \mathcal{T}\left(Z_{1}\right)=\underline{\mathfrak{B}}_{39} U_{1}\left(Z_{1}\right)=\mathbb{H}_{U_{1}}\left(Z_{1}\right) \cong h_{<1>}
$$


It also follows that the $\mathbf{B A}_{n}$-structure on $\left[\mathcal{T}\left(Z_{1}\right)\right]$ is induced by $\mathcal{T}$ from that on $\left[Z_{1}\right]$ so that we have a commutative diagram

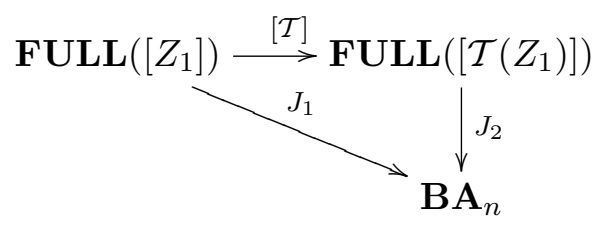

Taking into account (23) we get the following commutative diagram

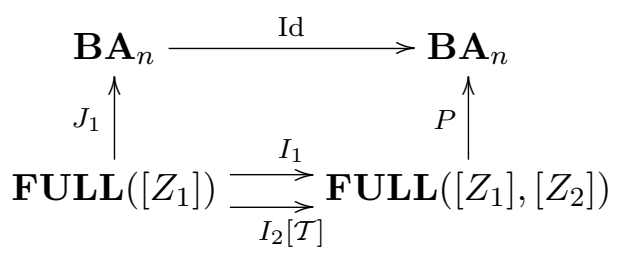

We can expand this diagram as follows

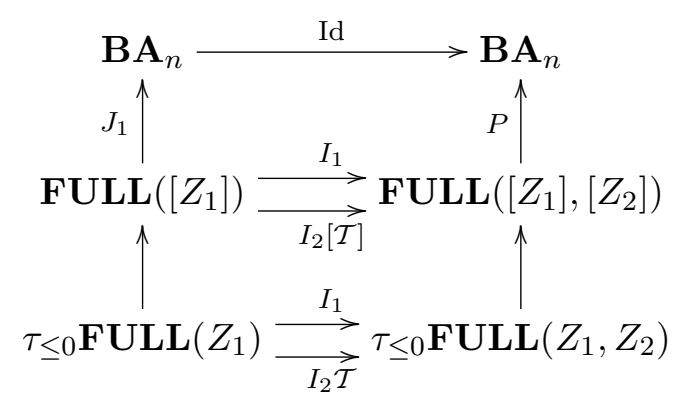

where the lower vertical arrows are just natural arrows that map $\tau_{\leq 0}$ of a complex to its zeroth cohomology. The composition of the left vertical arrows is a quasi-isomorphism: indeed, this composition equals to another composition:

$$
\tau_{\leq 0} \mathbf{F U L L}\left(Z_{1}\right) \rightarrow \tau_{\leq 0} \mathbf{F U L L}\left(\mathbb{H}\left(Z_{1}\right)\right) \rightarrow \mathbf{F U L L}\left(\left[\mathbb{H}\left(Z_{1}\right)\right]\right)
$$

$$
\cong \mathbf{F U L L}_{\mathbf{B A}_{n}^{\wedge}}\left(h_{<1>}\right)=\mathbf{B A}_{n}
$$

in which all arrows are SM weak equivalences .

It then easily follows that all the arrows in (24) are quasi-isomorphisms.

Next, we can produce the following diagram

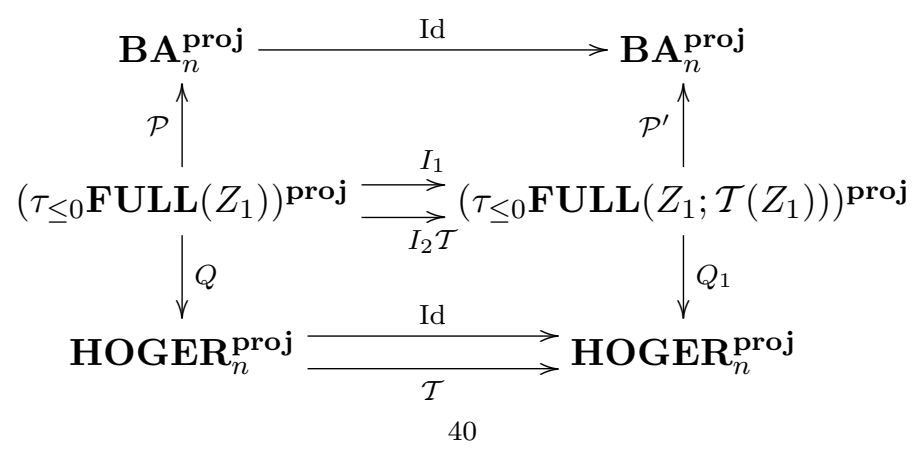


Where the maps $Q, Q_{1}$ are obvious embeddings which send $Z_{1}$ and $\mathcal{T}\left(Z_{1}\right)$ to themselves. Let us show that $Q$ is an equivalence. It is clear that $Q, Q_{1}$ induce a quasi-isomorphism on each complex of homomorphisms, we only need to check the essential surjectivity. As the image of $Q_{1}$ contains the image of $Q$, it suffices to check that $Q$ is an equivalence. It suffices to check that

$$
\mathbb{H} Q: \mathbf{F U L L}\left(Z_{1}\right)^{\text {proj }} \rightarrow \mathbf{F U L L}\left(\mathbb{H}\left(Z_{1}\right)\right)^{\text {proj }} \rightarrow \text { BA }_{n}^{\text {proj }}
$$

is an equivalence. This easily follows from the fact that $\mathbb{H}\left(Z_{1}\right) \cong h_{<1>}$. The argument is similar to that in (5.4.5)

Let $\mathcal{H} \in\left[\tau_{\leq 0} \mathbf{F U L L}(Z)\right]^{\text {proj }}$ be such that

$$
Q \mathcal{H} \cong h_{[1]}
$$

Such an $\mathcal{H}$ exists due to the fact that $Q$ is an equivalence.

The objects $\mathcal{P}^{\prime} I_{1} \mathcal{H} \cong \mathcal{P} \mathcal{H}$ and $\mathcal{P}^{\prime} I_{2} \mathcal{T}(\mathcal{H}) \cong \mathcal{P} \mathcal{H}$ are weakly equivalent, hence so are $I_{1} \mathcal{H}$ and $I_{2} \mathcal{T}(\mathcal{H})$. Let us choose this weak equivalence so that its image under $\mathcal{P}^{\prime}$ be the canonical isomorphism $\mathcal{P}^{\prime} I_{1} \mathcal{H} \cong \mathcal{P}^{\prime} I_{2} \mathcal{T}(\mathcal{H}) \cong \mathcal{P}(\mathcal{H})$.

Consider the following collection of data as in Sec. 8.2.2:

— the pair of maps $I_{1}, I_{2} \mathcal{T}$,

- the object $\mathcal{H}$ and the weak equivalence $I_{1} \mathcal{H} \rightarrow I_{2} \mathcal{T}(\mathcal{H})$.

These data produce a pair of arrows in $D(n$-op). Actually these arrows coincide. Indeed, it suffices to check this for the data obtained by applying the functors $\mathcal{P}, \mathcal{P}^{\prime}$ : we will get

- an object $\mathcal{P}(\mathcal{H}) \in \mathbf{B A}_{n}^{\text {free }}$;

- two coincident functors Id $=$ Id $: \mathbf{B A}_{n}^{\text {free }} \rightarrow \mathbf{B} \mathbf{A}_{n}^{\text {free }}$

— the identity quasi-isomorphism $\mathcal{P}(\mathcal{H}) \sim \operatorname{Id} \rightarrow \mathcal{P}(\mathcal{H})$.

These data do clearly produce a pair of coincident identity arrows in $D(n$-op).

Therefore, the following data produce coincident arrows in $D$ (n-op):

$$
\operatorname{HOGER}_{n}^{\text {proj }} \underset{\mathcal{T}}{\stackrel{\mathrm{Id}}{\longrightarrow}} \text { HOGER }_{n}^{\text {proj }}
$$

the object $Q(\mathcal{H}) \in$ HOGER $_{n}^{\text {proj }}$

the weak equivalence $Q(\mathcal{H}) \rightarrow \mathcal{T}(Q(\mathcal{H}))$ induced by the equivalence $I_{1} \mathcal{H} \rightarrow I_{2} \mathcal{T}(\mathcal{H})$.

Next, we have a quasi-isomorphism $h_{<1>} \rightarrow Q(\mathcal{H})$, hence a chain of quasi-isomorphisms

$$
h_{<1>} \rightarrow Q(\mathcal{H}) \rightarrow \mathcal{T} Q(\mathcal{H}) \rightarrow \mathcal{T} h_{<1>}=h_{\mathcal{T}(<1>)}=h_{<1>} .
$$

The composition of these arrows produces an element

$$
x \in H^{0} \operatorname{hom}_{\mathbf{H O G E R}}(<1>,<1>)=\mathbf{k} .
$$

This element must be invertible (i.e $x \neq 0$ ). This implies that the following data produce a pair of coincident arrows in $D(n$-op):

$$
\operatorname{hoger}_{\leq n} \underset{\mathcal{T}}{\stackrel{\mathrm{Id}}{\longrightarrow}} \text { hoger }_{\leq n}
$$

and a quasi-isomorphism

$$
<1>\rightarrow \mathcal{T}(<1>)=<1>
$$

which is the multiplication by $x$. 
This implies that the arrows $T: \operatorname{hoger}_{\leq n} \rightarrow$ hoger $_{\leq n}$ and

$$
\iota_{x}: \operatorname{hoger}_{\leq n}=\text { full }_{\text {HOGER }}(<1>)_{\leq n} \rightarrow \text { full }_{\text {HOGER }}(<1>)_{\leq n} \rightarrow \operatorname{hoger}_{\leq n}
$$

coincide in $D$ (n-op). This is a contradiction with our original assumption.

8.3. Dependence on the choice of an associator. As explained in Appendix 3, given an associator $\Phi$, we get an isomorphism $A_{\Phi} \in \mathrm{Iso}_{\mathrm{D}(\mathrm{op})}$ (braces, ger). Using the inverse isomorphism $\left(A_{\Phi}\right)^{-1}$ we then get a quantization functor $\mathbf{Q}_{\Phi}:=\mathbf{Q}_{\left(A_{\Phi}\right)^{-1}}$.

Question From [6] we can get another construction of a quantization functor, also using an associator. Does this Etingof-Kazhdan construction produce the quantization functor isomorphic to $\mathrm{Q}_{\Phi}$ ?

Theorem 11.4 from Appendix 3 implies that given different associators $\Phi_{1}$ and $\Phi_{2}$, we get $A_{\Phi_{1}} \nsim$ $A_{\Phi_{2}}$. Theorem 8.1 then readily implies

COROLLARY 8.2. Given different associators $\Phi_{1} \neq \Phi_{2}$, the corresponding quantization functors $\mathbf{Q}_{\Phi_{1}}, \mathbf{Q}_{\Phi_{2}}$ are non-isomorphic.

\section{Appendix 1: Categories}

9.0.1. Finite k-linear categories. A $k$-linear category is a category enriched over the category of $\mathbf{k}$-vector spaces.

Call such a category finite if the set of isomorphism classes of its objects is finite and the vector spaces of homomorphisms are finite dimensional for every pair of objects.

9.0.2. Given a finite category $\mathcal{C}$, let $\mathcal{C}^{\wedge}$ be the abelian category of $\mathbf{k}$-linear functors from $\mathcal{C}^{\text {op }}$ to the category of finite dimensional $\mathbf{k}$-vector spaces.

We have Ioneda's embedding $h: \mathcal{C} \rightarrow \mathcal{C}^{\wedge}$ defined by the formula $X \mapsto h_{X}$, where

$$
h_{X}(Y):=\operatorname{hom}_{\mathcal{C}}(Y, X) \text {. }
$$

9.1. DG-categories. Call a dg-category $\mathcal{C}$ finite if the set of isomorphism classes of its objects is finite and each complex $\operatorname{hom}_{\mathcal{C}}(X, Y)$ is finite (i.e. is bounded in both directions and each of its spaces is finitely dimensional).

Every finite $k$-linear category can be naturally viewed as a finite dg-category in which all homcomplexes are concentrated in degree 0 .

Let $\mathcal{C}^{\wedge \text { dg }}$ be the category of functors from $\mathcal{C}^{\text {op }}$ to the category of finite complexes. Given an $X \in \mathcal{C}$ we have $h_{X} \in \mathcal{C}^{\wedge \text { dg: }}$

whence Ioneda's embedding $\mathcal{C} \rightarrow \mathcal{C}^{\wedge \mathrm{dg}}$.

$$
h_{X}(Y):=\operatorname{hom}_{\mathcal{C}}(Y, X),
$$

9.1.1. Complexes. Given any dg-category $\mathcal{D}$ with a zero object, a complex in $\mathcal{D}$ is, by definition, a collection of objects $X_{n}, n \in \mathbb{Z}$ and elements $d_{n}: \in Z^{1} \operatorname{hom}\left(X_{n}, X_{n+1}\right)$ such that $d_{n+1} d_{n}=0$. A complex is called bounded if almost all of $X_{n}$ are zeros.

Given a complex $X^{\bullet}$ in $\mathcal{D}$ and an object $U \in \mathcal{D}$, we have a bi-complex of $\mathbf{k}$-vector spaces

$$
\cdots \operatorname{hom}\left(U, X^{n}\right) \stackrel{d_{n}}{\rightarrow} \operatorname{hom}\left(U, X^{n+1}\right) \stackrel{d_{n+1}}{\operatorname{hom}}\left(U, X^{n+2}\right) \rightarrow \cdots
$$

Denote by $h_{X} \bullet(U)$ the total complex of this bicomplex. This way we get a functor $h_{X} \bullet: \mathcal{D}^{\text {op }} \rightarrow$ complexes. If this functor is representable we denote the representing object by $\left|X^{\bullet}\right|$ and call it 
the realization of $X^{\bullet}$. Instead of saying that $h_{X} \bullet$ is representable, we will say that the complex $X^{\bullet}$ has a realization.

One sees that every bounded complex in $\mathcal{C}^{\wedge \text { dg }}$ has a realization.

9.1.2. We say that an $F \in \mathcal{C}^{\wedge \mathrm{dg}}$ is finitely generated and free if it is isomorphic to a finite direct sum of objects of the form $h_{X_{i}}\left[n_{i}\right]$, where $X_{i} \in \mathcal{C}$ and $\left[n_{i}\right]$ denotes a degree shift.

Let $\mathcal{C}^{\text {free }} \subset \mathcal{C}^{\wedge \mathrm{dg}}$ be the full sub-category consisting of all objects isomorphic to realizations of finite complexes of free finitely generated objects. Let $\mathcal{C}^{\text {proj }} \subset \mathcal{C}^{\wedge \mathrm{dg}}$ be the full subcategory consisting of all objects which are retractions of objects from $\mathcal{C}^{\text {free }}$. Alternatively, $\mathcal{C}^{\text {proj }}$ can be defined as the Karoubian closure of $\mathcal{C}^{\text {free }}$.

9.1.3. Induced functors. Let $\mathcal{C}, \mathcal{D}$ be a pair of finite dg categories and $F: \mathcal{C} \rightarrow \mathcal{D}$ be a functor.

The pre-composition with $F$ gives rise to a functor

$$
F^{-1}: \mathcal{D}^{\wedge \mathrm{dg}} \rightarrow \mathcal{C}^{\wedge \mathrm{dg}}
$$

This functor has a left adjoint

$$
F !: \mathcal{C}^{\wedge \mathrm{dg}} \rightarrow \mathcal{D}^{\wedge \mathrm{dg}}
$$

which is defined as follows. Define a functor

$$
\begin{aligned}
& H: \mathcal{C} \otimes \mathcal{D}^{\text {op }} \rightarrow \text { complexes; } \\
& H(X, Y)=\operatorname{hom}_{\mathcal{D}}(Y, F(X)) .
\end{aligned}
$$

Given $R \in \mathcal{C}^{\wedge \mathrm{dg}}$, that is $R: \mathcal{C}^{\text {op }} \rightarrow$ complexes, we set

$$
F_{!} R:=F \otimes_{\mathcal{C}} R
$$

9.1 .4 .

PROPOSITION 9.1. The functor $F_{!}$takes $\mathcal{C}^{\text {free }}$ to $\mathcal{D}^{\text {free }}$ and $\mathcal{C}^{\text {proj }}$ to $\mathcal{D}^{\text {proj }}$

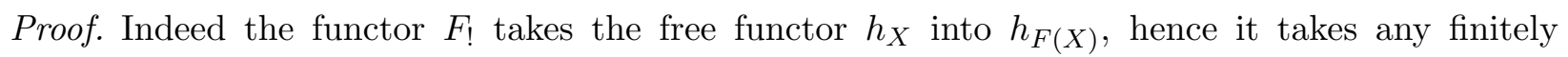
generated free functor to a finitely generated free functor and any finite complex of finitely generated free modules to a finite complex of finitely generated free modules. Next $F_{\text {! }}$ takes a retract $P \rightarrow G \rightarrow$ $P$ of finite complex $G$ of finitely generated free modules to a retract of $F_{!} G$, hence $F_{!} P \in \mathcal{D}^{\text {proj }}$.

9.1.5. Let us generalize the above construction as follows. Let $\mathcal{C}, \mathcal{D}$ be finite dg-categories; let $F$ be a functor $\mathcal{C} \rightarrow \mathcal{D}^{\wedge \mathrm{dg}}$. Define a functor $F_{!}: \mathcal{C}^{\wedge \mathrm{dg}} \rightarrow \mathcal{D}^{\wedge \mathrm{dg}}$ as follows. Let $K_{F}: \mathcal{C} \otimes \mathcal{D}^{\text {op }} \rightarrow$ complexes; $K_{F}(X, Y)=F(X)(Y)$. For $U \in \mathcal{C}^{\wedge \mathrm{dg}}$, set $F_{!} U:=F \otimes_{\mathcal{C}} K_{F}$.

9.1.6. One can prove that if $F: \mathcal{C} \rightarrow \mathcal{D}^{\text {free }}\left(\right.$ resp. $\left.F: \mathcal{C} \rightarrow \mathcal{D}^{\text {free }}\right)$, then $F_{!}\left(\mathcal{C}^{\text {free }}\right) \subset \mathcal{D}^{\text {free }}($ resp. $\left.F \mathcal{C}^{\text {proj }} \subset \mathcal{D}^{\text {proj }}\right)$. 
9.1.7. All these definitions make sense in the world of finite $\mathbf{k}$-linear categories. Let $\mathcal{C}, \mathcal{D}$ be $k$-linear categories and $F: \mathcal{C} \rightarrow \mathcal{D}$ be a $k$-linear functor. The functors

$$
F^{-1}: \mathcal{D}^{\wedge} \rightarrow \mathcal{C}^{\wedge}
$$

and

$$
F_{!}: \mathcal{C}^{\wedge} \rightarrow \mathcal{D}^{\wedge}
$$

can be defined in the same way as in the setting of dg-categories.

We define a finitely generated free object in $\mathcal{C}^{\wedge}$ as any object isomorphic to a finite direct sum of objects of the form $h_{X_{i}}$. We define a projective object as a retraction of a free object.

We then form full subcategories $\mathcal{C}^{f} \subset \mathcal{C}^{\text {pro }} \subset \mathcal{C}^{\wedge}$ consisting of free and projective objects respectively. In the same way as above, we see that the subcategories of free and projective objects are preserved by the functor $F$.

9.2. Symmetric monoidal categories. We will see that the above defined constructions work in the setting of symmetric monoidal categories.

9.2.1. We will use the notion of exterior product of dg-categories. Given a finite family of dgcategories $\mathcal{C}_{i}, i \in I$, we define their exterior product

$$
\bigotimes_{i \in I} \mathcal{C}_{i}
$$

by setting its objects to be arbitrary families $\bigotimes_{i \in I} X_{i}$, where $X_{i} \in \mathcal{C}_{i}$. Morphisms are defined as follows:

$$
\operatorname{hom}\left(\bigotimes_{i \in I} X_{i} ; \bigotimes_{i \in I} Y_{i}\right):=\bigotimes_{i \in I} \operatorname{hom}_{\mathcal{C}_{i}}\left(X_{i} ; Y_{i}\right)
$$

the composition of morphisms is defined in the obvious way.

We have natural functors

$$
\begin{aligned}
& \bigotimes_{i \in I} \mathcal{C}_{i}^{\wedge \mathrm{dg}} \rightarrow\left(\bigotimes_{i \in I} \mathcal{C}_{i}\right)^{\wedge \mathrm{dg}} ; \\
& \bigotimes_{i \in I} \mathcal{C}_{i}^{\wedge \mathrm{dg}} \rightarrow\left(\bigotimes_{i \in I} \mathcal{C}_{i}\right)^{\wedge \mathrm{dg}} ; \\
& \bigotimes_{i \in I} \mathcal{C}_{i}^{\text {free }} \rightarrow\left(\bigotimes_{i \in I} \mathcal{C}_{i}\right)^{\text {free }} \\
& \bigotimes_{i \in I} \mathcal{C}_{i}^{\text {proj }} \rightarrow\left(\bigotimes_{i \in I} \mathcal{C}_{i}\right)^{\text {proj }}
\end{aligned}
$$

All these functors send a family of functors $F_{i}: \mathcal{C}_{i}^{\text {op }} \rightarrow$ complexes to the following functor

$$
\begin{gathered}
F: \bigotimes_{i \in I} \mathcal{C}_{i} \rightarrow \text { complexes : } \\
F\left(\bigotimes_{i \in I} X_{i}\right):=\bigotimes_{i \in I} F_{i}\left(X_{i}\right) .
\end{gathered}
$$


9.2.2. Induced $S M$ structure on $\mathcal{C}^{\text {free }}, \mathcal{C}^{\text {proj }}, \mathcal{C}^{\wedge d g}$. Let $\mathcal{C}$ be a symmetric monoidal category. The tensor products give rise to functors

$$
\begin{gathered}
T^{I}: \mathcal{C}^{\bigotimes I} \rightarrow \mathcal{C}: \\
\bigotimes_{i \in I} X_{i} \mapsto \bigotimes_{i \in I} X_{i} .
\end{gathered}
$$

Whence induced functors

$$
\begin{gathered}
\left(\mathcal{C}^{\wedge \mathrm{dg}}\right)^{\bigotimes I} \rightarrow\left(\mathcal{C}^{\bigotimes I}\right)^{\wedge \mathrm{dg}} \stackrel{T_{!}^{I}}{\longrightarrow} \mathcal{C}^{\wedge \mathrm{dg}} \\
\left(\mathcal{C}^{\text {free }}\right)^{\bigotimes I} \rightarrow\left(\mathcal{C}^{\bigotimes I}\right)^{\text {free }} \stackrel{T_{!}^{I}}{\longrightarrow} \mathcal{C}^{\text {free }} \\
\left(\mathcal{C}^{\text {proj }}\right)^{\bigotimes I} \rightarrow\left(\mathcal{C}^{\bigotimes I}\right)^{\text {proj }} \stackrel{T_{!}^{I}}{\longrightarrow} \mathcal{C}^{\text {proj }}
\end{gathered}
$$

It is straightforward to check that these maps define an SM-structure on $\mathcal{C}^{\wedge \mathrm{dg}}, \mathcal{C}^{\text {free }}, \mathcal{C}^{\text {proj }}$.

The tensor product on $\mathcal{C}^{\text {free }}$ admits a more explicit description. Recall that any object of $\mathcal{C}^{\text {free }}$ is a realization of a bounded complex of finitely generated and free objects. Let us start with describing the tensor product of finitely generated and free objects. It is easy to see that we have

$$
\left(\bigoplus_{a \in A} h_{X_{a}}\left[n_{a}\right]\right) \otimes\left(\bigoplus_{b \in B} h_{X_{b}}\left[n_{b}\right]\right) \cong \bigoplus_{(a, b) \in A \times B} X_{a} \otimes Y_{b}\left[n_{a}+n_{b}\right]
$$

Next, given finite complexes of finitely generated and free objects, $X^{\bullet}$ and $Y^{\bullet}$, we see that

$$
\left|X^{\bullet}\right| \otimes\left|Y^{\bullet}\right|=\left|Z^{\bullet}\right|,
$$

where

$$
Z^{n}=\bigoplus_{m} X^{m} \otimes Y^{n-m}
$$

and the differential on $Z^{\bullet}$ is naturally induced by those on $X^{\bullet}, Y^{\bullet}$.

The tensor product on $\mathcal{C}^{\text {proj }}$ is uniquely defined by that on $\mathcal{C}^{\text {free }}$ and by the condition that the tensor product of kernels of projectors $P_{1}: X \rightarrow X$ and $P_{2}: Y \rightarrow Y$ is the kernel of the projector

$$
P_{1} \otimes \mathrm{Id}+\mathrm{Id} \otimes P_{2}: X \otimes Y \rightarrow X \otimes Y .
$$

9.2.3. Let $\mathcal{C}, \mathcal{D}$ be $\mathrm{SMC}$ and let $F: \mathcal{C} \rightarrow \mathcal{D}$ be a SM-functor. We will define a SM-structure on the induced functors

$$
\begin{aligned}
& F_{!}: \mathcal{C}^{\wedge \mathrm{dg}} \rightarrow \mathcal{D}^{\wedge \mathrm{dg}} \\
& F_{!}: \mathcal{C}^{\text {free }} \rightarrow \mathcal{D}^{\text {free }} \\
& F_{!}: \mathcal{C}^{\text {proj }} \rightarrow \mathcal{D}^{\text {proj }}
\end{aligned}
$$

Let us first of all reformulate the SM-structure on $F$ in a way convenient for us. Let $T_{\mathcal{C}}^{2}: \mathcal{C} \otimes \mathcal{C} \rightarrow$ $\mathcal{C} ; T_{\mathcal{D}}^{2}: \mathcal{D} \otimes \mathcal{D} \rightarrow \mathcal{D}$ be the tensor products. Part of the tensor structure on $F$ is an isomorphism

$$
T_{\mathcal{D}}^{2}(F \otimes F) \cong F T_{\mathcal{C}}^{2} .
$$

Let us now proceed to defining an SM-structure on $F$ !

Given $X, Y \in \mathcal{C}^{\wedge \mathrm{dg}}$, we are supposed to define isomorphisms

$$
F_{!}(X) \otimes F_{!}(Y) \rightarrow F_{!}(X \otimes Y) .
$$

The LHS is isomorphic to

$$
\left[T_{\mathcal{D}}^{2}(F \otimes F)\right] !(X \otimes Y) ;
$$


the RHS is isomorphic to

$$
\left[F T_{2}^{\mathcal{C}}\right]_{!}(X \otimes Y)
$$

The desired isomorphism (26) then comes from the isomorphism (25). It is straightforward to check that these isomorphisms satisfy all the properties of an SM-structure on a functor.

By the same token, one gets a SMS on the functors $F_{!}: \mathcal{C}^{\text {free }} \rightarrow \mathcal{D}^{\text {free }} ; F_{!}: \mathcal{C}^{\text {proj }} \rightarrow \mathcal{D}^{\text {proj }}$.

9.2.4. In the same way, given $\mathrm{SM}$ functors $F: \mathcal{C} \rightarrow \mathcal{D}^{\wedge \mathrm{dg}}$ (resp. $F: \mathcal{C} \rightarrow \mathcal{D}^{\text {free}}$; resp. $F: \mathcal{C} \rightarrow$ $\mathcal{D}^{\text {proj }}$ ), one gets a SM-structure on the induced functors

$$
F_{!}: \mathcal{C}^{\text {free }} \rightarrow \mathcal{D}^{\text {free }}, \text { resp. } \quad F_{!}: \mathcal{C}^{\text {proj }} \rightarrow \mathcal{D}^{\text {proj }}
$$

\section{Appendix 2: Derived Automorphisms of ger}

According to [10] there exists a closed model structure on the category of dg-operads. Therefore, one can construct the derived category of dg-operads following [17]. Let us denote this derived category by $\mathrm{D}(\mathrm{op})$. In this section we will study the automorphism group $\mathrm{Aut}_{\mathrm{D}(\mathrm{op})}$ (ger). We will see that it is isomorphic to an extension of $\mathbf{k}^{\times} \times \mathbf{k}^{\times}$by a pro-unipotent group, the latter group will be identified with the exponential group of a certain pro-nilpotent Lie algebra. We will conclude by showing that, roughly speaking, any homotopy non-trivial automorphism of hoger induces a homotopy non-trivial automorphism of the $N$-truncation hoger $_{\leq N}$ for $N$ large enough; see Sec. 10.1 for the definitions and a precise statement of the result.

10.0.5. Let $\mathbf{o}$ be a dg-operad. Quillen offers the following recipe for computing the set hom $\mathrm{D}_{\mathrm{op})}$ (ger, o) in the derived category of dg-operads.

Let hoger $\rightarrow$ ger be the standard resolution. Let $k[t, d t]$ be the polynomial commutative algebra on two generators $t$ of degree 0 and $d t$ of degree 1 with the differential sending $t$ to $d t$. Let $r_{0}, r_{1}: \mathbf{k}[t, d t] \rightarrow \mathbf{k}$ be the maps defined by $r_{0}(d t)=r_{1}(d t)=0 ; r_{0}(t)=0 ; r_{1}(t)=1$.

Let $\mathbf{o}[t, d t]$ be the dg-operad obtained from $\mathbf{o}$ by the extension of scalars $\mathbf{o}[t, d t](n):=\mathbf{o}(n) \otimes_{\mathbf{k}}$ $\mathbf{k}[t, d t]$. The maps $r_{0}, r_{1}$ induce maps of operads

$$
r_{0}, r_{1}: \mathbf{o}[t, d t] \rightarrow \mathbf{o}
$$

in the obvious way.

The set $\operatorname{hom}_{\mathrm{D}(\mathrm{op})}(\mathbf{g e r}, \mathbf{o})$ is identified with the quotient of the set hom(hoger, o) (in the usual category of dg-operads) by the following equivalence relation: two maps $f, g:$ hoger $\rightarrow$ o are equivalent iff there exists a map $h:$ hoger $\rightarrow \mathbf{o}[t, d t]$ (a homotopy) such that $r_{0} h=f ; r_{1} h=g$.

The set $\operatorname{hom}_{\mathrm{D}(\mathrm{op})}$ (hoger, hoger) is then a monoid and we would like to describe its group of invertible elements $\mathfrak{G}:=\operatorname{Aut}_{\mathrm{D}(\mathrm{op})}($ hoger$)$.

10.0.6. Given $\phi \in \mathfrak{G}$ it is represented by a map $f:$ hoger $\rightarrow$ hoger, and we can consider the induced map $\bar{f}$ : ger $=H^{\bullet}($ hoger $) \rightarrow H^{\bullet}($ hoger $)=$ ger. It follows that

1) $\bar{f}$ only depends on $\phi$, not on the choice of a representative $f$, so that we can denote $\bar{\phi}:=\bar{f}$;

2) the map $\phi \mapsto \bar{\phi}$ is a group homomorphism $\mathfrak{G} \rightarrow \operatorname{Aut}($ ger $)$.

On the other hand, the group Aut(ger) is isomorphic to $\mathbf{k}^{\times} \times \mathbf{k}^{\times}$. Given $(x, y) \in \mathbf{k}^{\times} \times \mathbf{k}^{\times}$, the corresponding automorphism of ger is given by dilating the commutative product by $x$ and the Lie bracket by $y$. It is clear that this way we get all automorphisms of ger, whence an isomorphism $\mathbf{k}^{\times} \rightarrow \operatorname{Aut}($ ger $)$.

Thus, we have a map

$$
\mathfrak{G} \rightarrow \operatorname{Aut}(\text { ger })=\mathbf{k}^{\times} \times \mathbf{k}^{\times}
$$


Next, we have a natural map $\operatorname{Aut}($ ger $) \rightarrow \mathfrak{G}$. Indeed, hoger is obtained by applying the co-bar construction to the shifted operad ger $\{1\}$, which is the Koszul dual to ger. Therefore, the action of $\mathbf{k}^{\times} \times \mathbf{k}^{\times}$on ger gives rise to an action of the same group on $\operatorname{ger}\{1\}$ and on hoger. One can check that the through map

$$
\mathbf{k}^{\times} \times \mathbf{k}^{\times} \rightarrow \mathfrak{G} \rightarrow \mathbf{k}^{\times} \times \mathbf{k}^{\times}
$$

is the identity, therefore, we have an extension

$$
\mathfrak{G}=\left(\mathbf{k}^{\times} \times \mathbf{k}^{\times}\right) \ltimes \mathfrak{G}_{0},
$$

where $\mathfrak{G}_{0} \subset \mathfrak{G}$ consists of all equivalence classes of automorphisms of hoger which induce the identity on $H^{\bullet}$ (hoger) = ger.

Below, we will find a pro-nilpotent Lie algebra whose exponential is narurally isomorphic to $\mathfrak{G}_{0}$.

10.0.7. We have a natural grading on hoger: Let $\operatorname{Gr}^{k} \operatorname{hoger}(n) \subset \operatorname{hoger}(n)$ be the span of all elements which can be expressed as a $(k-1)$-fold composition of the generators.

It is clear that $\mathrm{Gr}$ is compatible with the operadic composition on hoger and that the differential increases the grading by 1 .

Introduce a decreasing filtration on hoger:

$$
F^{k} \operatorname{hoger}(n):=\bigoplus_{l \geq k} \operatorname{Gr}^{l} \operatorname{hoger}(n) .
$$

As $\operatorname{Gr}{ }^{\geq n} \operatorname{hoger}(n)=0$, the direct sum is finite.

Any map of operads $f:$ hoger $\rightarrow$ hoger preserves this filtration. We say that $|f| \geq l$ if $(f-\mathrm{Id}) F^{k} \operatorname{hoger}(n) \subset F^{k+l} \operatorname{hoger}(n)$ It follows that $|f| \geq 0$ for all maps of operads $f$.

LEMMA 10.1. Suppose that $f:$ hoger $\rightarrow$ hoger represents an element from $\mathfrak{G}_{0}$ (or, which is the same, induces the identity $\operatorname{map} H^{\bullet}$ (hoger) $\rightarrow H^{\bullet}$ (hoger). Then $|f| \geq 1$.

Proof. Suppose it is not true that $|f| \geq 1$. Let $M$ be the minimal number such that the map

$$
f-\mathrm{Id}: \operatorname{hoger}(M) \rightarrow \operatorname{hoger}(M)
$$

does not increase filtration by 1 .

Let us show that $M>2$. Indeed the complex hoger(2) has zero differential, therefore, since $f$ induces the identity on the cohomology, the map $f: \operatorname{hoger}(2) \rightarrow \operatorname{hoger}(2)$ must be the identity map.

Thus, $M>2$. Let $G(k) \subset \operatorname{hoger}(k)$ be the spaces of generators.

Let $\mathbf{o}_{k} \subset$ hoger be the sub-operad generated by $\operatorname{hoger}(l), l \leq k$ (or, equivalently, by all $G(l)$, $l \leq k$.

It then follows that $\mathbf{o}_{k}$ is freely generated over $\mathbf{o}_{k-1}$ by $G(k)$ and that $d(G(k)) \subset \mathbf{o}_{k-1}(k)$. Let $D_{k}: G(k) \rightarrow \mathbf{o}_{k-1}(k)$ be the corresponding map.

Consider the map

$$
\phi_{M}: G(M) \stackrel{i_{M}}{\rightarrow} \operatorname{hoger}(M) \stackrel{f}{\rightarrow} \operatorname{hoger}(M),
$$

where $i_{M}$ is the inclusion of the space of generators $G(M)$ into $\operatorname{hoger}(M)$.

The compatibility with the differential implies that

$$
d \phi_{M} \pm \phi D=0 ;
$$

or

$$
d\left(\phi_{M}-i_{M}\right) \pm(\phi-\mathrm{Id}) D=0 .
$$


The map $D$ increases the filtration by 1 , as well as $\phi-\mathrm{Id}$, because $\phi-\operatorname{Id}: \operatorname{hoger}(m) \rightarrow \operatorname{hoger}(m)$ increases filtration by 1 for all $m<M$ by assumption. Thus, $d\left(\phi_{M}-i_{M}\right) \in F^{2} \operatorname{hoger}(M)$. This implies that the following composition is zero:

$$
G(M) \stackrel{\phi_{M}-i_{M}}{\longrightarrow} \operatorname{hoger}(M) \rightarrow \operatorname{Gr}^{0} \operatorname{hoger}(M) \stackrel{d}{\rightarrow} \operatorname{Gr}^{1} \operatorname{hoger}(M)
$$

On the other hand, it is easy to see that the rightmost arrow in this sequence is injective: assuming the contrary, every non-zero element $x \in \operatorname{Gr}^{0} \operatorname{hoger}(M)=G(M)$ such that $d x=0$ produces a nontrivial cycle in hoger $(M)$, on the other hand the through map $G(M) \rightarrow \operatorname{hoger}(M) \rightarrow \operatorname{ger}(M)$ is zero for all $M>2$. This contradicts to quasi-isomorphicity of the canonical map hoger $(M) \rightarrow$ $\operatorname{ger}(M)$.

Therefore, $\left(\phi_{M}-i_{M}\right)\left(G_{M}\right) \subset F^{1} \operatorname{hoger}(M)$. Since $\operatorname{hoger}(M)=\mathbf{o}_{M-1}(M) \oplus G(M)$, this readily implies that the map $f-\operatorname{Id}: \operatorname{hoger}(M) \rightarrow \operatorname{hoger}(M)$ increases the filtration by 1 , which is a contradiction.

10.0.8. Extend the grading on hoger to that on hoger $[t, d t]$ by setting the grading of $t$ to be 0 and the grading of $d t$ to be 1 . We then see that the operadic composition preserves this grading and that the grading of the differential $d$ is 1 .

Introduce a filtration

$$
F^{n} \operatorname{hoger}[t, d t](m):=\bigoplus_{N \geq n} \operatorname{Gr}^{N} \operatorname{hoger}[t, d t](m)
$$

Note that the direct sum here is actually finite.

Every map of operads $f:$ hoger $\rightarrow$ hoger $[t, d t]$ preserves this filtration. We write $|f| \geq k$ if $(f-\mathrm{Id}): \operatorname{hoger}(m) \rightarrow \operatorname{hoger}(m)$ increases the filtration by at least $k$.

LEMMA 10.2. Let $f:$ hoger $\rightarrow$ hoger $[t, d t]$ be such that $\left|r_{0} f\right| \geq 1$. Then $|f| \geq 1$.

Proof. Let us choose an $m$ and decompose $f: \operatorname{hoger}(m) \rightarrow \operatorname{hoger}[t, d t](m)$ as

$$
\sum_{k}\left(u_{k}+v_{k} d t\right) t^{k}
$$

where $u_{k}, v_{k}: \operatorname{hoger}(m) \rightarrow \operatorname{hoger}(m)$. It follows that both $u_{k}, v_{k}$ preserve the filtration on $\operatorname{hoger}(m)$.

The equality $d f=0$ implies that $k u_{k}+d v_{k-1}=0$. Therefore, for all $k \geq 1, u_{k}$ increases the filtration by 1 , because so does $d$ and $v_{k-1}$ preserves the filtration.

Next, $u_{0}=r_{0} f$, therefore, $u_{0}-$ Id increases the filtration by 1 .

Lastly, $v_{k} t^{k} d t$ increases the filtration by 1 as so does $d t$. This means that $|f| \geq 1$.

10.0.9. Let $K$ be the group of all maps $f: \operatorname{hoger} \rightarrow \operatorname{hoger}[t, d t]$ for which $|f| \geq 1$. Let $G$ be the group of all maps $f:$ hoger $\rightarrow$ hoger with $|f| \geq 1$.

The maps $r_{0}, r_{1}:$ hoger $[t, d t] \rightarrow$ hoger induce group homomorphisms $r_{0}, r_{1}: K \rightarrow G$. Next, we have a group homomorphism

$$
p: G \rightarrow \mathfrak{G}_{0}
$$

which is surjective in virtue of Lemma 10.1 .

Lemma 10.2 implies that $p\left(x_{0}\right)=p\left(x_{1}\right)$ iff there exists a $y \in K$ such that $x_{0}=r_{0}(y) ; x_{1}=r_{1}(y)$. 
10.0.10. Let $\mathfrak{k}$ be the DGLA of $\mathbf{k}[t, d t]$-linear derivations of hoger $[t, d t]$. The filtration on hoger $[t, d t]$ induces that on $\mathfrak{k}$ and it follows that $\mathfrak{k}=F^{0} \mathfrak{k}$. It follows that $\mathfrak{k}$ is complete with respect to this filtration and that $F^{1 \mathfrak{k}} \subset \mathfrak{k}$ is a nilpotent ideal. Let $Z^{0} F^{1} \mathfrak{k} \subset F^{1 \mathfrak{k}}$ be the Lie sub-algebra of zero-cycles. This is a pro-nilpotent Lie algebra so that we can form a group

$$
\operatorname{Exp}\left(Z^{0} F^{1 \mathfrak{k}}\right)
$$

This group acts on hoger by automorphisms which are congruent to 1 modulo $F^{1}$, whence a map

$$
\operatorname{Exp}\left(Z^{0} F^{1} \mathfrak{k}\right) \rightarrow K
$$

We have the inverse logarithm map showing that the map (27) is a group isomorphism.

Analogously, let $\mathfrak{g}$ be the DGLA of derivations of the operad hoger. It follows that $\mathfrak{k}=\mathfrak{g}[t, d t]:=$ $\mathfrak{g} \otimes_{\mathbf{k}} \mathbf{k}[t, d t]$. In a similar way, we get an isomorphism

$$
\operatorname{Exp}\left(Z^{0} F^{1} \mathfrak{g}\right) \rightarrow G .
$$

The maps $r_{0}, r_{1}: K \rightarrow G$ are induced by the DGLA maps

$$
r_{0}, r_{1}: \mathfrak{k}=\mathfrak{g}[t, d t] \rightarrow \mathfrak{g}
$$

induced by the maps $r_{0}, r_{1}: \mathbf{k}[t, d t] \rightarrow \mathbf{k}[t]$.

We see that two elements

$$
e^{X_{0}}, e^{X_{1}} \in \operatorname{Exp}\left(Z^{0} F^{1} \mathfrak{g}\right)=G
$$

go to the same element in $\mathfrak{G}_{0}$ iff there is a $Y \in Z^{0} F^{1} \mathfrak{k}$ such that $r_{0} Y=X_{0}, r_{1} Y=X_{1}$. It is easy to see that such a $Y$ exists iff $X_{0}-X_{1}=d Z$ for some $Z \in \mathfrak{g}$, i.e. if $X_{0}-X_{1}$ is a boundary. Let $\mathfrak{b} \subset Z^{0} F^{1} \mathfrak{g}$ be the ideal formed by all elements $d Z, Z \in \mathfrak{g}^{-1}$ (note that since $d$ increases filtration by $\left.1, d Z \in F^{1} \mathfrak{g}\right)$. We then get that $\operatorname{Exp}(\mathfrak{b}) \subset \operatorname{Exp}\left(Z^{0} F^{1} \mathfrak{g}\right)$ is the kernel of the projection $G \rightarrow \mathfrak{G}_{0}$, whence an isomorphism

$$
\operatorname{Exp}\left(Z^{0} F^{1} \mathfrak{g} / \mathfrak{b}\right) \rightarrow \mathfrak{G}_{0}
$$

Lastly, we have an identification

$$
Z^{0} F^{1} \mathfrak{g} / \mathfrak{b}=F^{1} H^{0}(\mathfrak{g})
$$

Thus we have proven:

THEOREM 10.3. We have a natural isomorphism

$$
\operatorname{Exp}\left(F^{1} H^{0}(\mathfrak{g})\right) \rightarrow \mathfrak{G}_{0} .
$$

\subsection{Truncations of the operad hoger.}

10.1.1. Truncated operads. Define an n-truncated operad $\mathbf{o}$ in a $S M C \mathcal{C}$ as

- a functor from the groupoid of finite sets with at most $n-1$ elements to $\mathcal{C}$;

— given a map of finite sets $f: S \rightarrow T$ with $|S|,|T|<n$, there should be given a composition map

$$
\mathbf{o}(T) \otimes \bigotimes_{t \in T} \mathbf{o}\left(f^{-1} t\right) \rightarrow \mathbf{o}(S)
$$

- the composition maps should be associative in the same way as in the setting of usual operads

Given a usual operad $\mathcal{A}$; its spaces $\mathcal{A}(S),|S| \leq n$ form an $n$-truncated operad. Denote this truncated operad by $\mathcal{A}_{\leq n}$ The category of $n$-truncated dg-operads has a closed model structure. Hence, we can consider the derived category, to be denoted by $D(n$-op). We have an obvious 
functor $F_{n}: \mathrm{D}(\mathrm{op}) \rightarrow D(n$-op $)$. The object ger $\in \mathrm{D}(\mathrm{op})$ is of our particular interest, and we have natural homomorphisms

$$
O_{n}: \operatorname{Aut}_{\mathrm{D}(\mathrm{op})}(\text { ger }) \rightarrow \operatorname{Aut}_{D(n-\mathrm{op})} \text { ger }_{\leq n}
$$

Let $\mathfrak{G}_{0}^{\leq n} \subset \operatorname{Aut}_{D(n \text {-op })}$ be the subgroup consisting of all elements inducing the identity of $H^{\bullet}\left(\right.$ ger $\left._{\leq n}\right)$. We then have homomorphisms

$$
O_{n}: \mathfrak{G}_{0} \rightarrow \mathfrak{G}_{0}^{\leq n} .
$$

We want to prove:

PROPOSITION 10.4. For every $X \in \mathfrak{G}_{0}, X \neq I$, there exists an $n$ such that $O_{n}(X) \neq I$.

In order to prove this theorem we need to rewrite $\mathfrak{G}_{0}^{\leq n}$ in terms of Lie algebras, in the same way as we did it with $\mathfrak{G}_{0}$.

Let $\mathfrak{g}^{\geq n}$ be the DGLA of derivations of the operad hoger $_{\leq n}$. We have a grading and a filtration on hoger $_{\leq n}$ in the same way as on hoger, so that we have an induced grading and filtration on $\mathfrak{g}^{\geq n}$. We then have an identification $\mathfrak{G}_{0}^{\leq n}=\operatorname{Exp}\left(F^{1} H^{0}\left(\mathfrak{g}^{\leq n}\right)\right)$ in the same way as for $\mathfrak{G}_{0}$. The proof is similar and is omitted.

The map $O_{n}: \mathfrak{G}_{0} \rightarrow \mathfrak{G}_{0}^{\leq n}$ is induced by the natural map $o_{n}: \mathfrak{g} \rightarrow \mathfrak{g}^{\leq n}$. So the proposition reduces to:

LEMMA 10.5. For every $X \in F^{1} H^{0}(\mathfrak{g}), X \neq 0$, there exists an $n$ such that $o_{n}(X) \neq 0$.

We have a grading on $\mathfrak{g} ; \mathfrak{g}=\prod_{n} \operatorname{Gr}^{n} \mathfrak{g}$. The differential increases the grading by 1 . Therefore, the complex $\mathfrak{g}$ splits as

$$
\mathfrak{g}=\prod_{n} \mathfrak{g}^{(n)}
$$

where $\mathfrak{g}^{(n)}$ is the following complex:

$$
\cdots \rightarrow\left(\mathrm{Gr}^{n} \mathfrak{g}\right)^{0} \rightarrow\left(\mathrm{Gr}^{n+1} \mathfrak{g}\right)^{1} \rightarrow \cdots
$$

Same splitting takes place for $\mathfrak{g}_{\leq N}$ so that we have

$$
\mathfrak{g}_{\leq N}=\prod_{n} \mathfrak{g}_{\leq N}^{(n)}
$$

and the map $\mathfrak{g} \rightarrow \mathfrak{g}_{\leq N}$ is induced by maps

$$
\mathfrak{g}^{(n)} \rightarrow \mathfrak{g}_{\leq N}^{(n)}
$$

One sees that these maps are surjective. Let $\Phi^{N}\left(\mathfrak{g}^{(n)}\right)$ be the kernel of (28) We see that $\Phi$ is a filtration on the complex $\mathfrak{g}^{(n)}$ and that $\Phi^{N} \mathfrak{g}^{(n)} \subset \mathfrak{g}^{n}$ is the subcomplex consisting of all derivations vanishing on $\mathbf{o}_{N} \subset$ hoger. From this one sees that $\Phi$ is a complete filtration. We have associated graded complexes $\operatorname{Gr}_{\Phi}^{m} \mathfrak{g}^{(n)}$. We have:

$$
\left(\operatorname{Gr}_{\Phi}^{m} \mathfrak{g}^{(n)}\right)^{k} \cong \operatorname{hom}^{k}\left(G(m) ; \operatorname{Gr}^{k+n} \operatorname{hoger}(m)\right) .
$$

The differential is induced by the differential on hoger:

$$
d: \operatorname{Gr}^{k+n} \operatorname{hoger}(m) \rightarrow \operatorname{Gr}^{k+1+n} \operatorname{hoger}(m)
$$

The latter differential is acyclic unless $k+n \neq m-1$.

Thus, $H^{k}\left(\operatorname{Gr}_{\Phi}^{m} \mathfrak{g}^{(n)}\right)=0$ unless $k=m-1-n$. 
Since the filtration $\Phi$ is complete we have a spectral sequence $E_{2}^{m, k-m}:=H^{k}\left(\mathrm{Gr}_{\Phi}^{m} \mathfrak{g}^{(n)}\right) \Rightarrow$ $H^{k}\left(\mathfrak{g}^{(n)}\right)$ in which the differentials $d_{r}, r \geq 2$ are zero. This implies that the map

$$
H^{k}\left(\mathfrak{g}^{(n)}\right) \rightarrow H^{k}\left(\mathfrak{g}^{(n)} / \Phi^{N} \mathfrak{g}^{(n)}\right)
$$

is isomorphisms for all $N \geq n+k$.

In particular, the maps

$$
H^{0}\left(\mathfrak{g}^{(n)}\right) \rightarrow H^{0}\left(\mathfrak{g}_{\leq N}^{(n)}\right)
$$

are isomorphisms for all $N \geq n$.

Given an $X \in F^{1} H^{0}(\mathfrak{g})=\prod_{n \geq 1} H^{0}\left(\mathfrak{g}^{(n)}\right), X \neq 0$ there exists an $n \geq 1$, such that the component $X^{(n)} \in H^{0}\left(\mathfrak{g}^{(n)}\right)$ is not zero, hence the image of $X$ in $F^{1} H^{0}\left(\mathfrak{g}_{\leq N}\right)$ is non-zero for all $N \geq n$.

10.1.2. Let $\iota_{x}:$ hoger $\rightarrow$ hoger be as in (19). This way we get maps

$$
\iota_{N}: \mathbf{k}^{\times} \stackrel{\iota}{\rightarrow} \operatorname{Aut}_{\mathrm{D}(\mathrm{op})}(\text { ger }) \rightarrow \operatorname{Aut}_{D(n-\mathrm{op})}\left(\text { ger }_{\leq n}\right) .
$$

PROPOSITION 10.6. For every $X \in \mathfrak{G}, X \notin \iota\left(\mathbf{k}^{\times}\right)$, there exists an $n$ such that $O_{n}(X) \notin \iota_{n}\left(\mathbf{k}^{\times}\right)$.

Proof. Consider the image $\left(x_{1}, x_{2}\right) \in \mathbf{k}^{\times} \times \mathbf{k}^{\times}$of $X$ under the map

$$
\mathfrak{G} \rightarrow \mathbf{k}^{\times} \times \mathbf{k}^{\times} .
$$

Note that the latter map factors as:

$$
\mathfrak{G} \rightarrow \mathfrak{G}_{n} \rightarrow \mathbf{k}^{\times} \times \mathbf{k}^{\times}
$$

Therefore, if $x_{1} \neq x_{2}$, the statement of the theorem is true for all $n$. Thus, $x_{1}=x_{2}=x$ and we have $Z:=\iota_{x}^{-1} X \in \mathfrak{G}_{0}$. Since $X$ is not in the image of $\iota\left(\mathbf{k}^{\times}\right.$, we conclude that $Z \neq \mathrm{Id}$. Therefore, according to Proposition 10.4, the image of $Z$ in $\mathfrak{G}_{n}$ is not identity.

On the other hand, the image of $Z$ under the projection $\mathfrak{G}_{n} \rightarrow \mathbf{k}^{\times} \times \mathbf{k}^{\times}$is the identity. This implies that the image of $X=\iota_{x} Z$ in $\mathfrak{G}_{n}$ is not equal to $\iota_{n}(x)$ for any $x \in \mathbf{k}^{\times}$.

\section{Appendix 3: Associators and GT}

We only collect the information that is needed in this paper. The reader can find expositions of the theory of the associator and the GT group in the original paper [5], see also [3], [21].

For the purposes of the present paper, we only need to know the following facts:

11.0.3. Given an associator $\Phi$ over $\mathbf{k}$, one has a canonical element $A_{\Phi} \in \mathrm{Iso}_{\mathrm{D}(\mathrm{op})}$ (braces, ger). The construction is as follows.

1) proofs of Deligne's conjecture in [19] [13] [20] provide us with a zigzag quasi-isomoprhism of the operad braces with the operad of singular chains of the topological operad of little disks.

2) In [16], given an associator $\Phi$, we construct a zigzag quasi-isomorphism between the chain operad of little disks and the operad of Gerstenhaber algebras.

Combining 1)-2) we get a zigzag quasi-isomorphism between the operads braces and ger. This zigzag defines element $A_{\Phi} \in \mathrm{Iso}_{\mathrm{D}(\mathrm{op})}$ (braces, ger).

Let us recall the construction. In [3] the associator is essentially defined as any isomorphism between two operads, $\mathbf{P a B}$ ans $\mathbf{P a C D}$ in the category of small categories, using the standard nerve and simplicial chain functors, one obtains an induced isomorphism of dg- operads

$$
C \bullet(N(\mathbf{P a B})) \underset{51}{\stackrel{\Phi_{*}}{\rightarrow}} C_{\bullet}(N(\mathbf{P a C D})) .
$$


Denote the operad on the LHS by $\mathbf{o}_{1}$ and the operad on the RHS by $\mathbf{o}_{2}$, so that we have an isomorphism

$$
B(\Phi): \mathbf{o}_{1} \rightarrow \mathbf{o}_{2}
$$

Lastly, one constructs:

a) a zigzag quasi-isomorphism between $\mathbf{o}_{1}$ and the singular chain operad of the little disks (hence, by 1) with the operad braces);

b) a zigzag quasi-isomorphism between $\mathbf{o}_{2}$ and ger

11.0.4. GRT. In [3] the group GRT defined as an automorphism group of the operad PaCD, hence a free and transitive GRT-action on the set of all associators and an action of GRTon $\mathbf{O}_{2}$, because the operad $\mathbf{o}_{2}$ is obtained from PaCD in a functorial way.

Given a $g \in \mathbf{G R T}$ and an associator $\Phi$, let $g . \Phi$ be the result of the action of $\mathfrak{g}$ on $\Phi$. Let us also denote by $g_{*}: \mathbf{o}_{2} \rightarrow \mathbf{o}_{2}$ the automorphism induced by $g$. We then get $B_{g . \Phi}=g_{*} B_{\Phi}$.

A zig-zag quasi-isomorphism of $\mathbf{o}_{2}$ and ger gives rise to a canonical isomorphism $\mathbf{o}_{2} \rightarrow$ ger in the category $\mathrm{D}(\mathrm{op})$. Therefore, the GRT-action on $\mathbf{O}_{2}$ canonically defines a map

$$
T: \mathbf{G R T} \rightarrow \operatorname{Aut}_{\mathrm{D}(\mathrm{op})}(\text { ger })=\mathfrak{G}
$$

It easily follows that $A(g . \Phi)=T(g) A(\Phi)$.

11.0.5. The group GRT is known to be an extension of $\mathbf{k}^{\times}$by a prounipotent group $\mathbf{G R T}_{0}$

From the previous subsection, we have a map GRT $\rightarrow \mathfrak{G}$. The through map

$$
\mathbf{G R T}_{0} \rightarrow \mathbf{G R T} \rightarrow \mathfrak{G} \rightarrow \mathbf{k}^{\times} \times \mathbf{k}^{\times}
$$

must be the identity, as easily follows from the theory of algebraic groups. Therefore, we get an induced map $\mathbf{G R T}_{0} \rightarrow \mathfrak{G}_{0}$. This map produces a map of the Lie algebras

$$
\mathfrak{g} r t_{0} \rightarrow F^{1} H^{0}(\mathfrak{g})
$$

In $[16]$ we show that the latter map is injective, therefore

THEOREM 11.1. The map of exponentials $\mathbf{G R T}_{0} \rightarrow \mathfrak{G}_{0}$ is also injective.

The map of the quotients

$$
\mathbf{k}^{\times}=\mathbf{G R T} / \mathbf{G R T}_{0} \rightarrow \mathfrak{G} / \mathfrak{G}_{0}=\mathbf{k}^{\times} \times \mathbf{k}^{\times}
$$

can be also proven to be injective. Furthermore, we have:

LEMMA 11.2. The map (30) sends $x \in \mathbf{k}^{\times}$to $(1, x) \in \mathbf{k}^{\times} \times \mathbf{k}^{\times}$

Proof. The category PaCD is equivalent to the category with one object whose endomorphism space is the completed universal enveloping algebra of abelian one dimensional Lie algebra. Denote the generator of this Lie algebra by $t$. The action of GRT on $\mathbf{P a C D}(2)$ factors through the projection $p: \mathbf{G R T} \rightarrow \mathbf{k}^{\times}$so that $g \in \mathbf{G R T}$ dilates $t$ by $p(g): g . t=\pi(g) t$.

The operad $C_{\bullet}(\mathbf{P a C D}(2))$ is canonically quasi-isomorphic to Chevalley-Eilenberg chain complex $C_{-\bullet}(\mathfrak{t})$, where $\mathfrak{t}$ is the one dimensional Lie algebra generated by $t$. This complex has one-dimensional zeroth and one dimensional negative first cohomology so that the cohomology of $C_{-} \bullet(t)$ is canonically identified with ger $(2)$.

The induced GRT-action on this cohomology (from the action on $\mathfrak{t}$ by dilations) can be easily found to be trivial on the zeroth cohomology; the action on the negative first cohomology is by dilations, whence the statement. 
We can now make the following statement.

Consider the diagonal embedding $\mathbf{k}^{\times} \rightarrow \mathbf{k}^{\times} \times \mathbf{k}^{\times}$. Let

$$
\iota: \mathbf{k}^{\times} \rightarrow \mathbf{k}^{\times} \times \mathbf{k}^{\times} \rightarrow \mathfrak{G}
$$

be the through map. Let $\mathfrak{G}^{\prime}: \mathfrak{G} / \iota\left(\mathbf{k}^{\times}\right)$be the quotient.

We then have the through map:

$$
\mathbf{G R T} \stackrel{29}{\rightarrow} \mathfrak{G} \rightarrow \mathfrak{G}^{\prime}
$$

THEOREM 11.3. The map (31) is injective

Proof. Indeed, the the induced maps

$$
\mathbf{G R T}_{0} \rightarrow \mathfrak{G}_{0} \rightarrow \mathfrak{G}^{\prime}
$$

and

$$
\mathbf{k}^{\times}=\mathbf{G R T} / \mathbf{G R T}_{0} \rightarrow \mathfrak{G}^{\prime} / \mathfrak{G}_{0}=\mathbf{k}^{\times}
$$

are both injective: the arrow $\mathbf{G R T}_{0} \rightarrow \mathfrak{G}_{0}$ by Theorem 11.1 ; the arrow $\mathfrak{G}_{0} \rightarrow \mathfrak{G}^{\prime}$ is injective by inspection; the arrow GRT/GRT $\mathbf{G}_{0} \rightarrow \mathfrak{G}^{\prime} / \mathfrak{G}_{0}$ is an isomorphism as follows from Lemma 11.2.

11.0.6. Let us now study elements $A_{\Phi_{1}}, A_{\Phi_{2}} \in \mathrm{Iso}_{\mathrm{D}(\mathrm{op})}$ (braces, ger).

We have a map $\iota: \mathbf{k}^{\times} \rightarrow \mathfrak{G}=\operatorname{Aut}_{\mathrm{D}(\mathrm{op})}($ ger $)$. Call two elements $U, V \in \mathrm{Iso}_{\mathrm{D}(\mathrm{op})}$ (braces, ger) equivalent if they are conjugated by the action of an element $\iota(x)$ for some $x \in \mathbf{k}^{\times}$.

We can now prove

THEOREM 11.4. Given two different associators $\Phi_{1}$ and $\Phi_{2}$, the elements

$$
A_{\Phi_{1}}, A_{\Phi_{2}} \in \mathrm{Iso}_{D(o p)}(\text { braces, ger })
$$

are not equivalent.

Proof. We have $\Phi_{2}=g . \Phi_{1}$ for some $g \in \mathbf{G R T}, g \neq e$. Let $\bar{g} \in \mathfrak{G}$ be the image of $g$. We know that $\bar{g} \notin \iota\left(\mathbf{k}^{\times}\right)$by Theorem 11.3.

Next, $A_{\Phi_{2}}=\bar{g} \cdot A_{\Phi_{1}}$. As $\bar{g} \notin \iota\left(\mathbf{k}^{\times}\right)$, it follows that $A_{\Phi_{2}}$ and $A_{\Phi_{1}}$ are non-equivalent.

Acknowledgements The author would like to thank P. Etingof, E. Getzler, D. Kazhdan, and B. Tsygan for fruitful discussions and numerous useful advice. The author greatly appreciates useful remarks by the referee.

\section{REFERENCES}

[1] A. Beilinson, J. Bernstein, P. Deligne, Faisceaux Pervers, Asterisque 1982 Vol. 100

[2] A. I. Bondal, M. M. Kapranov, Framed triangulated categories, Math. USSR-Sb. 70 (1991), no. 1, 93-107

[3] D. Bar-Natan, On associators and the Grothendieck-Teichmuller group. I. Selecta Math. (N.S.) 4 (1998), no. 2, 183-212.

[4] H. Cartan and S. Eilenberg, Methods of Homological Algebra

[5] V. Drinfeld, On quasitriangular quasi-Hopf algebras and a group closely related to $\operatorname{Gal}(\overline{\mathbb{Q}} / \mathbb{Q})$

[6] P. Etingof and D. Kazhdan, Quantization of Lie bialgebras, I, Selecta Math. 2(1996) n. 1 1-41.

[7] B. Enriques and P. Etingof, On the invertibility of quantization functors, math.QA/0306212

[8] A. Voronov; M. Gerstenhaber, Higher-order operations on the Hochschild complex. (Russian) Funktsional. Anal. i Prilozhen. 29 (1995), no. 1, 1-6, 96; translation in Funct. Anal. Appl.29 (1995), no. 1, 1-5

[9] E. Getzler,J. D. S. Jones, Operads, Homotopy algebra, and Iterated integrals for double loop spaces, hepth/940355...

[10] V. Hinich, Homological algebra of homotopy algebras. Comm. Algebra 25 (1997), no. 10, 3291-3323. 
[11] V. Hinich, Tamarkin's proof of Kontsevich formality theorem. Forum Math. 15 (2003), no. 4, 591-614

[12] M. Kontsevich, Operads and motives in deformational quantization, Lett. Math. Phys 48 (1999) no. 1, 35-72

[13] M. Kontsevich and Y. Soibelman, Deformation of algerbas over operads and Deligne's conjecture, Conférence Moshé Flato 1999, vol. 1. Math. Phys. Studies 21, Kluwer Acad. Publ., Dordrecht, 2000, pp. 255-307

[14] M. Markl, A resolution (miminal model) of the PROP for bialgebras, math.AT/0209007

[15] D.Tamarkin, Formality of the chain operad of small squares, math.QA/ 9809164

[16] D. Tamarkin, Action of the Grotendieck-Teichmüller group on the operad of Gerstenhaber algebras, math.QA/0202039

[17] D. Quillen, Rational homotopy theory. Ann. of Math. (2) 901969 205-295.

[18] Deformations of Lie bialgebras via the formality of the operad of little disks, Deformation quantization (Strasbourg, 2001), 203-236, IRMA Lect. Math. Theor. Phys., 1, de Gruyter, Berlin, 2002

[19] J. McClure, J. Smith. A solution of Deligne's Hochschild cohomology conjecture. Recent progress in homotopy theory (Baltimore, MD, 2000), 153-193, Contemp. Math., 293, Amer. Math. Soc., Providence, RI, 2002.

[20] A. Voronov, Homotopy Gerstenhaber algebras. Confrence Mosh Flato 1999, Vol. II (Dijon), 307-331,Math. Phys. Stud., 22, Kluwer Acad. Publ., Dordrecht, 2000

[21] P. Etingof, O. Schiffmann, Lectures on quantum groups. Lectures in Mathematical Physics. International Press, Boston, MA, 1998. 239 pp 